\title{
Real Gromov-Witten Theory in All Genera and Real Enumerative Geometry: Computation
}

\author{
Penka Georgieva* and Aleksey Zinger ${ }^{\dagger}$
}

October 13, 2018

\begin{abstract}
The first part of this work constructs real positive-genus Gromov-Witten invariants of realorientable symplectic manifolds of odd "complex" dimensions; the second part studies the orientations on the moduli spaces of real maps used in constructing these invariants. The present paper applies the results of the latter to obtain quantitative and qualitative conclusions about the invariants defined in the former. After describing large collections of real-orientable symplectic manifolds, we show that the real genus 1 Gromov-Witten invariants of sufficiently positive almost Kahler threefolds are signed counts of real genus 1 curves only and thus provide direct lower bounds for the counts of these curves in such targets. We specify real orientations on the real-orientable complete intersections in projective spaces; the real Gromov-Witten invariants they determine are in a sense canonically determined by the complete intersection itself, (at least) in most cases. We also obtain equivariant localization data that computes the real invariants of projective spaces and determines the contributions from many torus fixed loci for other complete intersections. Our results confirm Walcher's predictions for the vanishing of these invariants in certain cases and for the localization data in other cases.
\end{abstract}

\section{Contents}

1 Introduction 2

1.1 Real-orientable symplectic manifolds . . . . . . . . . . . . . . . . 3

1.2 Gromov-Witten and enumerative invariants . . . . . . . . . . . . . 5

1.3 Real invariants of complete intersections . . . . . . . . . . . . . . . 6

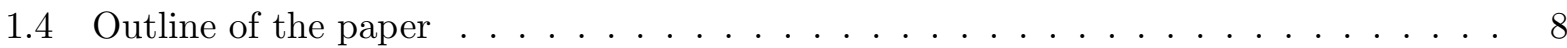

2 Some examples 9

2.1 Real orientable symplectic manifolds . . . . . . . . . . . . . . . . 9

2.2 Real orientations on complete intersections . . . . . . . . . . . . . . . 14

2.3 The canonical orientations of the moduli spaces . . . . . . . . . . . 15

3 Proof of Theorem $1.5 \quad 21$

3.1 Real GW- and enumerative invariants . . . . . . . . . . . . . . . 21

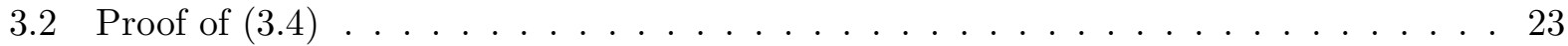

*Partially supported by ERC grant STEIN-259118

${ }^{\dagger}$ Partially supported by NSF grants DMS 1500875 and MPIM 
4 Equivariant localization

4.1 Equivariant setting . . . . . . . . . . . . . . . . . . 28

4.2 Equivariant localization data . . . . . . . . . . . . . . . . 31

4.3 Examples and applications . . . . . . . . . . . . . . . 37

5 Proof of Theorem 4.6

5.1 The torus-fixed loci . . . . . . . . . . . . . . . . . . 42

5.2 The fixed-locus contribution . . . . . . . . . . . . . . . . 46

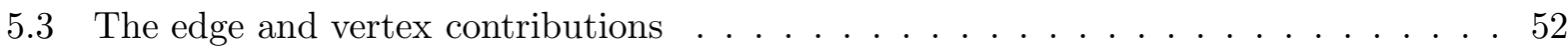

5.4 Proof of Proposition $5.5 \ldots \ldots \ldots \ldots \ldots \ldots \ldots$

\section{Introduction}

The theory of $J$-holomorphic maps plays prominent roles in symplectic topology, algebraic geometry, and string theory. The foundational work of [17, 24, 30, 20, 5] has established the theory of (closed) Gromov-Witten invariants, i.e. counts of $J$-holomorphic maps from closed Riemann surfaces to symplectic manifolds. In [11], we introduce the notion of real orientation on a real symplectic manifold $(X, \omega, \phi)$. A real orientation on a real symplectic $2 n$-manifold $(X, \omega, \phi)$ with $n \notin 2 \mathbb{Z}$ induces orientations on the moduli spaces of real $J$-holomorphic maps from arbitrary genus $g$ symmetric surfaces to $(X, \phi)$ commuting with the involutions on the domain and the target and thus gives rise to arbitrary-genus real Gromov-Witten invariants, i.e. counts of such maps, for real-orientable symplectic manifolds of odd "complex" dimension $n$. These orientations are studied in detail in [12]. The present paper applies the results of [12] to the computation of these invariants.

Propositions 1.2 and 1.4 provide large collections of real-orientable symplectic manifolds; they include the odd-dimensional projective spaces and the quintic threefold, which plays a special role in the interactions of Gromov-Witten theory with string theory. We also show that the genus 1 real Gromov-Witten invariants of sufficiently positive almost Kahler threefolds are signed counts of real genus 1 curves only; see Theorem 1.5. The "classical" Gromov-Witten invariants in contrast include genus 0 contributions and generally are not integer; see (1.6). Thus, the genus 1 real Gromov-Witten invariants provide direct lower bounds for the counts of real genus 1 curves in many almost Kahler threefolds.

An explicit real orientation on each complete intersection $X_{n ; \mathbf{a}}$ of Proposition 1.4 is specified in Section 2.2. This real orientation may depend on the parametrization of the ambient projective space with its involution, on the ordering of the line bundles corresponding to $X_{n ; \mathbf{a}}$, and on the section $s_{n ; \mathbf{a}}$ cutting out $X_{n ; \mathbf{a}}$ (i.e. negating some components of $s_{n ; \mathbf{a}}$ may change the induced real orientation). However, we show that the real Gromov-Witten invariants of a fixed complete intersection $X_{n ; \mathbf{a}}$ determined by this real orientation are independent of all these choices in most cases and vanish in the subset of these cases predicted in [32]; see Theorem 1.6. In the cases not covered by the independence statement of this theorem, the real Gromov-Witten invariants are expected to vanish as well. These invariants are also preserved by linear inclusions of the ambient projective space into larger projective spaces; see Proposition 2.6. Furthermore, the signed count of real lines through a pair of conjugate points in $\mathbb{P}^{2 m-1}$ with the standard conjugation is +1 with respect to these orientations:

$$
\left\langle H^{2 m-1}\right\rangle_{0,1}^{2 m-1}, \tau_{2 m}=+1 .
$$


Section 4.2 describes the equivariant localization data that computes the contributions to these invariants from many torus fixed loci. If $X_{n ; \mathbf{a}}=\mathbb{P}^{2 m-1}$ (but $g$ is arbitrary) or $g=0$ (but $X_{n ; \mathbf{a}}$ is arbitrary), this description covers all fixed loci and thus completely determines the real GromovWitten invariants in these cases. Theorem 4.6] is used in [27] to compute the real genus $g$ degree $d$ Gromov-Witten invariants of $\mathbb{P}^{3}$ with $d$ conjugate pairs of point constraints for $g \leqslant 5$ and $d \leqslant 8$. The equivariant localization data of Section 4.2 agrees with [32, (3.22)]. This implies that the spin structure on the real locus of the quintic threefold $X_{5 ;(5)}$ used, but not explicitly specified, in [28] is the spin structure associated with our real orientation on $\left(X_{5 ;(5)}, \tau_{5 ;(5)}\right)$.

\subsection{Real-orientable symplectic manifolds}

An involution on a topological space $X$ is a homeomorphism $\phi: X \longrightarrow X$ such that $\phi \circ \phi=\mathrm{id}_{X}$. By an involution on a manifold, we will mean a smooth involution. Let

$$
X^{\phi}=\{x \in X: \phi(x)=x\}
$$

denote the fixed locus. An anti-symplectic involution $\phi$ on a symplectic manifold $(X, \omega)$ is an involution $\phi: X \longrightarrow X$ such that $\phi^{*} \omega=-\omega$. For example, the maps

$$
\begin{aligned}
\tau_{n}: \mathbb{P}^{n-1} & \longrightarrow \mathbb{P}^{n-1}, \\
\eta_{2 m}: \mathbb{P}^{2 m-1} & \longrightarrow \mathbb{P}^{2 m-1}, \quad\left[Z_{1}, \ldots, Z_{n}\right] \longrightarrow\left[\bar{Z}_{1}, \ldots, \bar{Z}_{n}\right], \\
& \longrightarrow\left[\bar{Z}_{2},-\bar{Z}_{1}, \ldots, \bar{Z}_{2 m},-\bar{Z}_{2 m-1}\right],
\end{aligned}
$$

are anti-symplectic involutions with respect to the standard Fubini-Study symplectic forms $\omega_{n}$ on $\mathbb{P}^{n-1}$ and $\omega_{2 m}$ on $\mathbb{P}^{2 m-1}$, respectively. If

$$
k \geqslant 0, \quad \mathbf{a} \equiv\left(a_{1}, \ldots, a_{k}\right) \in\left(\mathbb{Z}^{+}\right)^{k},
$$

and $X_{n ; \mathbf{a}} \subset \mathbb{P}^{n-1}$ is a complete intersection of multi-degree a preserved by $\tau_{n}$, then $\left.\tau_{n ; \mathbf{a}} \equiv \tau_{n}\right|_{X_{n ; \mathbf{a}}}$ is an anti-symplectic involution on $X_{n ; \mathbf{a}}$ with respect to the symplectic form $\omega_{n ; \mathbf{a}}=\left.\omega_{n}\right|_{X_{n ; \mathbf{a}}}$. Similarly, if $X_{2 m ; \mathbf{a}} \subset \mathbb{P}^{2 m-1}$ is preserved by $\eta_{2 m}$, then $\left.\eta_{2 m ; \mathbf{a}} \equiv \eta_{2 m}\right|_{X_{2 m ; \mathbf{a}}}$ is an anti-symplectic involution on $X_{2 m ; \mathbf{a}}$ with respect to the symplectic form $\omega_{2 m ; \mathbf{a}}=\left.\omega_{2 m}\right|_{X_{2 m ; \mathbf{a}}}$. A real symplectic manifold is a triple $(X, \omega, \phi)$ consisting of a symplectic manifold $(X, \omega)$ and an anti-symplectic involution $\phi$.

Let $(X, \phi)$ be a topological space with an involution. A conjugation on a complex vector bundle $V \longrightarrow X$ lifting an involution $\phi$ is a vector bundle homomorphism $\widetilde{\phi}: V \longrightarrow V$ covering $\phi$ (or equivalently a vector bundle homomorphism $\tilde{\phi}: V \longrightarrow \phi^{*} V$ covering $\mathrm{id}_{X}$ ) such that the restriction of $\tilde{\phi}$ to each fiber is anti-complex linear and $\widetilde{\phi} \circ \widetilde{\phi}=\mathrm{id}_{V}$. $\underset{\widetilde{\phi}}{\mathrm{A}}$ real bundle pair $(V, \widetilde{\phi}) \longrightarrow(X, \phi)$ consists of a complex vector bundle $V \longrightarrow X$ and a conjugation $\widetilde{\phi}$ on $V$ lifting $\phi$. For example,

$$
\left(X \times \mathbb{C}^{n}, \phi \times \mathfrak{c}\right) \longrightarrow(X, \phi),
$$

where $\mathfrak{c}: \mathbb{C}^{n} \longrightarrow \mathbb{C}^{n}$ is the standard conjugation on $\mathbb{C}^{n}$, is a real bundle pair. If $X$ is a smooth manifold, then $(T X, \mathrm{~d} \phi)$ is also a real bundle pair over $(X, \phi)$. For any real bundle pair $(V, \widetilde{\phi})$ over $(X, \phi)$, we denote by

$$
\Lambda_{\mathbb{C}}^{\text {top }}(V, \widetilde{\phi})=\left(\Lambda_{\mathbb{C}}^{\text {top }} V, \Lambda_{\mathbb{C}}^{\text {top }} \widetilde{\phi}\right)
$$

the top exterior power of $V$ over $\mathbb{C}$ with the induced conjugation. Direct sums, duals, and tensor products over $\mathbb{C}$ of real bundle pairs over $(X, \phi)$ are again real bundle pairs over $(X, \phi)$. 
Definition 1.1 ([11, Definition 5.1]). Let $(X, \phi)$ be a topological space with an involution and $(V, \varphi)$ be a real bundle pair over $(X, \phi)$. A real orientation on $(V, \varphi)$ consists of

(RO1) a rank 1 real bundle pair $(L, \widetilde{\phi})$ over $(X, \phi)$ such that

$$
w_{2}\left(V^{\varphi}\right)=w_{1}\left(L^{\widetilde{\phi}}\right)^{2} \quad \text { and } \quad \Lambda_{\mathbb{C}}^{\text {top }}(V, \varphi) \approx(L, \widetilde{\phi})^{\otimes 2},
$$

(RO2) a homotopy class of isomorphisms of real bundle pairs in (1.3), and

(RO3) a spin structure on the real vector bundle $V^{\varphi} \oplus 2\left(L^{*}\right)^{\tilde{\phi}^{*}}$ over $X^{\phi}$ compatible with the orientation induced by (RO2).

An isomorphism in (1.3) restricts to an isomorphism

$$
\Lambda_{\mathbb{R}}^{\text {top }} V^{\varphi} \approx\left(L^{\tilde{\phi}}\right)^{\otimes 2}
$$

of real line bundles over $X^{\phi}$. Since the vector bundles $\left(L^{\widetilde{\phi}}\right)^{\otimes 2}$ and $2\left(L^{*}\right)^{\tilde{\phi}^{*}}$ are canonically oriented, (RO2) determines orientations on $V^{\varphi}$ and $V^{\varphi} \oplus 2\left(L^{*}\right)^{\widetilde{\phi}^{*}}$. By the first assumption in (1.3), the real vector bundle $V^{\varphi} \oplus 2\left(L^{*}\right)^{\tilde{\phi}^{*}}$ over $X^{\phi}$ admits a spin structure.

Let $(X, \omega, \phi)$ be a real symplectic manifold. A real orientation on $(X, \omega, \phi)$ is a real orientation on the real bundle pair $(T X, \mathrm{~d} \phi)$. We call $(X, \omega, \phi)$ real-orientable if it admits a real orientation. The next three statements, which are established in Section 2.1, describe large collections of real-orientable symplectic manifolds.

Proposition 1.2. Let $(X, \omega, \phi)$ be a real symplectic manifold with $w_{2}\left(X^{\phi}\right)=0$. If

(1) $H_{1}(X ; \mathbb{Q})=0$ and $c_{1}(X)=2\left(\mu-\phi^{*} \mu\right)$ for some $\mu \in H^{2}(X ; \mathbb{Z})$ or

(2) $X$ is compact Kahler, $\phi$ is anti-holomorphic, and $\mathcal{K}_{X}=2\left([D]+\left[\overline{\phi_{*} D}\right]\right)$ for some divisor $D$ on $X$,

then $(X, \omega, \phi)$ is a real-orientable symplectic manifold.

Corollary 1.3. Let $n \in \mathbb{Z}^{+}$and $\mathbf{a} \equiv\left(a_{1}, \ldots, a_{n-4}\right) \in\left(\mathbb{Z}^{+}\right)^{n-4}$ be such that

$$
a_{1}+\ldots+a_{n-4} \equiv n \quad \bmod 4 .
$$

If $X_{n ; \mathbf{a}} \subset \mathbb{P}^{n-1}$ is a complete intersection of multi-degree a preserved by $\tau_{n}$, then $\left(X_{n ; \mathbf{a}}, \omega_{n ; \mathbf{a}}, \tau_{n ; \mathbf{a}}\right)$ is a real-orientable symplectic manifold.

Proposition 1.4. Let $m, n \in \mathbb{Z}^{+}, k \in \mathbb{Z}^{\geqslant 0}$, and $\mathbf{a} \equiv\left(a_{1}, \ldots, a_{k}\right) \in\left(\mathbb{Z}^{+}\right)^{k}$.

(1) If $X_{n ; \mathbf{a}} \subset \mathbb{P}^{n-1}$ is a complete intersection of multi-degree a preserved by $\tau_{n}$,

$$
a_{1}+\ldots+a_{k} \equiv n \quad \bmod 2, \quad \text { and } \quad a_{1}^{2}+\ldots+a_{k}^{2} \equiv a_{1}+\ldots+a_{k} \quad \bmod 4
$$

then $\left(X_{n ; \mathbf{a}}, \omega_{n ; \mathbf{a}}, \tau_{n ; \mathbf{a}}\right)$ is a real-orientable symplectic manifold. 
(2) If $X_{2 m ; \mathbf{a}} \subset \mathbb{P}^{2 m-1}$ is a complete intersection of multi-degree a preserved by $\eta_{2 m}$ and

$$
a_{1}+\ldots+a_{k} \equiv 2 m \quad \bmod 4
$$

then $\left(X_{2 m ; \mathbf{a}}, \omega_{2 m ; \mathbf{a}}, \eta_{2 m ; \mathbf{a}}\right)$ is a real-orientable symplectic manifold.

As indicated by the proof of Corollary 1.3 in Section 2.1 the condition $w_{2}\left(X^{\phi}\right)=0$ in Proposition 1.2 is redundant if $X$ is a threefold. In particular, every real compact Kahler Calabi-Yau threefold is real-orientable.

By Proposition 1.4 $\left(\mathbb{P}^{2 m-1}, \tau_{2 m}\right)$ and $\left(\mathbb{P}^{4 m-1}, \eta_{4 m}\right)$ are real-orientable symplectic manifolds. So are the complete intersection Calabi-Yau threefolds

$$
X_{5 ;(5)} \subset \mathbb{P}^{4}, \quad X_{6 ;(3,3)} \subset \mathbb{P}^{5}, \quad \text { and } \quad X_{8 ;(2,2,2,2)} \subset \mathbb{P}^{7}
$$

preserved by the standard conjugation on the ambient space. Proposition 1.4](1) does not apply to the two remaining projective complete intersection Calabi-Yau threefolds,

$$
X_{6 ;(2,4)} \subset \mathbb{P}^{5} \quad \text { and } \quad X_{7 ;(2,2,3)} \subset \mathbb{P}^{6},
$$

as they do not satisfy the second conditions in (1.5). These two threefolds are instead real-orientable by Corollary 1.3, whenever they are preserved by the standard conjugation on the ambient space. In contrast to Proposition 1.4](1), Corollary 1.3 does not endow the symplectic manifolds to which it applies with a natural real orientation.

\subsection{Gromov-Witten and enumerative invariants}

A symmetric surface $(\Sigma, \sigma)$ is a closed oriented (possibly nodal) surface $\Sigma$ with an orientationreversing involution $\sigma$. If $\Sigma$ is smooth, the fixed locus $\Sigma^{\sigma}$ of $\sigma$ is a disjoint union of circles. If in addition $(X, \phi)$ is a manifold with an involution, a real map

$$
u:(\Sigma, \sigma) \longrightarrow(X, \phi)
$$

is a smooth map $u: \Sigma \longrightarrow X$ such that $u \circ \sigma=\phi \circ u$.

For a symplectic manifold $(X, \omega)$, we denote by $\mathcal{J}_{\omega}$ the space of $\omega$-compatible almost complex structures on $X$. If $\phi$ is an anti-symplectic involution on $(X, \omega)$, let

$$
\mathcal{J}_{\omega}^{\phi}=\left\{J \in \mathcal{J}_{\omega}: \phi^{*} J=-J\right\} .
$$

For a genus $g$ symmetric surface $(\Sigma, \sigma)$, possibly nodal and disconnected, we similarly denote by $\mathcal{J}_{\Sigma}^{\sigma}$ the space of complex structures $\mathfrak{j}$ on $\Sigma$ compatible with the orientation such that $\sigma^{*} \mathfrak{j}=-\mathfrak{j}$. For $J \in \mathcal{J}_{\omega}^{\phi}$, a real $J$-holomorphic map consists of a symmetric surface $(\Sigma, \sigma), \mathfrak{j} \in \mathcal{J}_{\Sigma}^{\sigma}$, and a real $(J, \mathfrak{j})$-holomorphic map $u: \Sigma \longrightarrow X$.

Let $(X, \omega, \phi)$ be a real-orientable symplectic $2 n$-manifold with $n \notin 2 \mathbb{Z}, g, l \in \mathbb{Z} \geqslant 0, B \in H_{2}(X ; \mathbb{Z})$, and $J \in \mathcal{J}_{\omega}^{\phi}$. We denote by $\overline{\mathfrak{M}}_{g, l}(X, B ; J)^{\phi}$ the moduli space of equivalence classes of stable real degree $B$ $J$-holomorphic maps from genus $g$ symmetric (possibly nodal) surfaces with $l$ conjugate pairs of 
marked points. By [11, Theorem 1.4], a real orientation on $(X, \omega, \phi)$ determines an orientation on this compact space, endows it with a virtual fundamental class, and thus gives rise to real genus $g$ GW-invariants of $(X, \omega, \phi)$ that are independent of the choice of $J \in \mathcal{J}_{\omega}^{\phi}$. If $n=3$ and $c_{1}(X)$ is divisible by 4 , a real orientation on $(X, \omega, \phi)$ also determines a count of real genus $1 J$-holomorphic curves with conjugate and real point insertions; see [11, Theorem 1.5].

The real genus $g$ GW-invariants of [11, Theorem 1.4] are in general combinations of counts of real curves of genus $g$ and counts of real curves of lower genera, just as happens in the "classical" complex setting. In light of [36, Theorems 1A/B] and [39, Theorem 1.5], it seems plausible that integral counts of genus $g$ real curves alone can be extracted from these GW-invariants to directly provide lower bounds for enumerative counts of real curves in good situations. This would typically involve delicate obstruction analysis. However, the situation is fairly simple if $g=1$ and $n=3$. The statement below applies whenever it makes sense in the context of [11, Theorems 1.4,1.5]; see the beginning of Section 3 and (3.4).

Theorem 1.5. Let $(X, \omega, \phi)$ be a compact real-orientable 6 -manifold and $J \in \mathcal{J}_{\omega}^{\phi}$ be an almost complex structure which is genus 1 regular in the sense of [37, Definition 1.4]. The genus 1 real $G W$-invariants of $(X, \omega, \phi)$ are then equal to the corresponding signed counts of real $J$-holomorphic curves and thus provide lower bounds for the number of real genus 1 irreducible curves in $(X, J, \phi)$.

In contrast, the complex genus 1 degree $d$ enumerative and GW-invariants of $\mathbb{P}^{3}$ are related by the formula

$$
\mathrm{E}_{1, d}=\mathrm{GW}_{1, d}+\frac{2 d-1}{12} \mathrm{GW}_{0, d} .
$$

This formula, originally announced as Theorem A in 14], is established as a special case of [38, Theorem 1.1], comparing standard and "reduced" GW-invariants (the latter do not "see" the genus 0 curves in sufficiently positive cases).

The real genus $g \mathrm{GW}$-invariants of $(X, \omega, \phi)$ with $g \geqslant 2$, include counts of lower genus curves with rational coefficients, even if the real dimension of $X$ is 6 and $X$ is very positive. For sufficiently positive almost Kahler real-orientable manifolds $(X, \omega, \phi)$ of real dimension 6 , these contributions are determined in [26].

\subsection{Real invariants of complete intersections}

Explicit real orientations on the real-orientable complete intersections of Proposition 1.4 are described in Section 2.2. They in general depend on some auxiliary choices made. By studying the effect of these choices on the resulting orientations of the moduli spaces of real maps, we establish the following statement in Section 2.3 .

Theorem 1.6. Let $(X, \phi)$ denote an odd-dimensional complete intersection $\left(X_{n ; \mathbf{a}}, \omega_{n ; \mathbf{a}}, \tau_{n ; \mathbf{a}}\right)$ or $\left(X_{2 m ; \mathbf{a}}, \omega_{2 m ; \mathbf{a}}, \eta_{2 m ; \mathbf{a}}\right)$ satisfying the assumptions of Proposition 1.4 and $g, d \in \mathbb{Z} \geqslant 0$.

(1) If either $\phi=\eta_{n ; \mathbf{a}}$, or $k g=0$, or $g \notin 2 \mathbb{Z}$ and $d \in 2 \mathbb{Z}$, or $a_{i} \notin 2 \mathbb{Z}$ for all $i$ and $d-g \notin 2 \mathbb{Z}$, then the genus $g$ degree $d$ real $G W$-invariants of $(X, \phi)$ determined by the real orientation of Section 2.2 with insertions from $\mathbb{P}^{n-1}$ are independent of the real parametrization of the ambient projective space, the ordering of the line bundles associated with the complete intersection, and the section $s_{n ; \mathbf{a}}$ cutting out the complete intersection $(X, \phi)$. 
(2) Suppose the genus $g$ degree $d$ real $G W$-invariants of $(X, \phi)$ with insertions from $\mathbb{P}^{n-1}$ are independent of the real parametrization of the ambient projective space, the ordering of the line bundles associated with the complete intersection, and the section $s_{n ; \mathbf{a}}$. If either $d-g \in 2 \mathbb{Z}$ or $a_{i} \in 2 \mathbb{Z}$ for some $i$ and $g \in 2 \mathbb{Z}$, then these invariants vanish.

This theorem refers to the real GW-invariants of [11, Theorems 1.4,1.5]. The proof we give in Section 2.3 addresses the case of conjugate pairs of primary insertions (i.e. pullbacks of cohomology classes on $\mathbb{P}^{n-1}$ ), but it is easily adaptable to the descendant insertions (i.e. $\psi$-classes). It also applies to the real genus $1 \mathrm{GW}$-invariants with real point insertions under the conditions of [11, Theorem 1.5], because the number of real point insertions is then even and the appropriate analogue of the middle vertical arrow in (2.29) is thus still orientation-preserving. Theorems 1.5 and 1.6](2) imply that the genus 1 odd-degree complex enumerative invariants of $\mathbb{P}^{3}$ with pairs of the same insertions are even, as claimed in [11, Corollary 2.6].

The last assumption in Theorem 1.6](2) is that the real invariants of the complete intersections cut out by two different transverse real sections $s_{n ; \mathbf{a}}$ and $s_{n: \mathbf{a}}^{\prime}$ are the same (even if the complete intersections are not). The last conclusion of Theorem 1.6](1) is weaker, as the complete intersection $(X, \phi)$ is fixed. By the next paragraph, the stronger conclusion holds if $\phi=\eta_{n ; \mathbf{a}}$ or $k g=0$. We expect that it holds in all cases; see the next paragraph and Remark 2.4. The GW-invariants of $(X, \phi)$ are predicted in 32 to vanish in all cases not covered by the assumptions of Theorem 1.6](1). Conversely, the independence of the real GW-invariants of $(X, \phi)$ of all choices and in all cases would confirm the vanishing predictions of [32] in all cases.

In the case of the complete intersections $\left(X_{2 m ; \mathbf{a}}, \eta_{2 m ; \mathbf{a}}\right)$, the space of transverse sections is pathconnected; see the proof of Theorem 1.6. The construction of real orientations of Section 2.2 lifts over such paths. Thus, the real orientations on these complete intersections and the resulting real GW-invariants are independent of the choice of the section $s_{2 m ; \mathbf{a}}$ cutting out $X_{2 m ; \mathbf{a}}$. On the other hand, the topology of the real locus of a (smooth) complete intersection $\left(X_{n ; \mathbf{a}}, \tau_{n ; \mathbf{a}}\right)$ may depend on the choice of the section $s_{n ; \mathbf{a}}$ cutting out $X_{n ; \mathbf{a}}$. Thus, the real orientations of Section 2.2 for different complete intersections of multidegree $\mathbf{a}$ in $\left(\mathbb{P}^{n-1}, \tau_{n}\right)$ are not comparable in general. However, the resulting real GW-invariants can still be compared. In the case of the primary insertions arising from $\mathbb{P}^{n-1}$, these invariants are expected to be related to the sheaf $\mathcal{V}_{n ; \mathbf{a}}^{\widetilde{\phi}_{n ; \mathbf{a}}}$ in (4.1) in a manner independent of the section $s_{n ; \mathbf{a}}$. This is indeed the case for the $g=0$ invariants, as shown in [28] for $\left(X_{5 ;(5)}, \tau_{5 ;(5)}\right)$. Whenever the real GW-invariants of $X_{n ; \mathbf{a}}$ are related to $\mathcal{V}_{n ; \mathbf{a}}^{\widetilde{\phi}_{n ; \mathbf{a}}}$ as expected, they are also independent of the choice of the section $s_{n ; \mathbf{a}}$.

The projective spaces $\mathbb{P}^{n-1}$ and $\mathbb{P}^{2 m-1}$ admit natural, "half"-dimensional torus actions that are compatible with the involutions $\tau_{n}$ and $\eta_{2 m}$, respectively; these are the torus actions appearing in [32, Section 3]. We determine the torus fixed loci of the induced actions on the moduli spaces of real maps and their normal bundles in Sections 5.1 and 5.2, respectively. The resulting equivariant localization data, which is described in Section 4.2, determines the real genus $g$ GW-invariants of $\left(\mathbb{P}^{2 m-1}, \tau_{2 m}\right)$ and $\left(\mathbb{P}^{4 m-1}, \tau_{4 m}\right)$ with conjugate pairs of constraints via the virtual equivariant localization theorem of [15]. Since the moduli spaces of real maps into these targets are smooth in the genus 0 case and all torus fixed loci are contained in the smooth locus in the genus 1 case, the classical equivariant localization theorem of [1] suffices in these cases. We also determine the equiv- 
ariant contributions to the real GW-invariants of the real-orientable complete intersections $X_{n ; \mathbf{a}}$ that arise from the torus fixed loci containing no maps with contracted components of positive genus; see Theorem 4.6. In contrast to the usual approach in real GW-theory of choosing a halfgraph, our computational algorithm allows to pick the non-fixed edges and vertices at random (one from each conjugate pair); see Remark 4.7. Our equivariant localization data is consistent with [32, (3.22)]. We also obtain the two types of cancellations of contributions from some fixed loci predicted in the Calabi-Yau cases in [32, Sections 3.2,3.3]; see Corollary 4.8 and the second case of Lemma 5.3 .

By Theorem 1.5, the genus 1 real GW-invariants of $\left(\mathbb{P}^{3}, \omega_{4}, \tau_{4}\right)$ and $\left(\mathbb{P}^{3}, \omega_{4}, \eta_{4}\right)$ are lower bounds for the enumerative counts of such curves in $\left(\mathbb{P}^{3}, J_{0}, \tau_{4}\right)$ and $\left(\mathbb{P}^{3}, J_{0}, \eta_{4}\right)$, respectively. The lower bound for the number of real genus 1 degree $d$ curves passing through $d$ pairs of conjugate point insertions obtained from the equivariant localization computation of Section 4 is 0 for $d=2,1$ for $d=4$, and 4 for $d=6$; see Examples 4.10 and 4.11] and [13]. The $d=2$ number is as expected, since there are no connected degree 2 curves of any kind passing through two generic pairs of conjugate points in $\mathbb{P}^{3}$. The $d=4$ number is also not surprising, since there is only one genus 1 degree 4 curve passing through 8 generic points in $\mathbb{P}^{3}$; see the first three paragraphs of [19, Section 1]. By (1.6) and [7, the number of genus 1 degree 6 curves passing through 12 generic points in $\mathbb{P}^{3}$ is 2860 . Our signed count of -4 for the real genus 1 degree 6 curves through 6 pairs of conjugate points in $\mathbb{P}^{3}$ is thus consistent with the complex count and provides a non-trivial lower bound for the number of real genus 1 degree 6 curves with 6 pairs of conjugate point insertions.

In Section 4.3, we use the equivariant localization data of Section 4.2 to give an alternative proof of Theorem 1.6](2) in the case of projective spaces and to compare the real GW-invariants for the two involution types; see Proposition [1.7 below. The genus 0 and 1 cases of both of these statements appear in [3, Theorem 1.9] and [4, Theorem 7.2], respectively (the latter presumes that genus 1 real GW-invariants of $\mathbb{P}^{3}$ can be defined).

Proposition 1.7. The genus $g$ real $G W$-invariants of $\left(\mathbb{P}^{4 m-1}, \omega_{4 m}, \tau_{4 m}\right)$ and $\left(\mathbb{P}^{4 m-1}, \omega_{4 m}, \eta_{4 m}\right)$ with only conjugate pairs of insertions differ by the factor of $(-1)^{g-1}$.

The proof of Proposition 1.7 extends directly to the real GW-invariants of the complete intersections $(X, \phi)$ of Theorem 1.6 whenever they are related to the sheaf $\mathcal{V}_{n ; \mathbf{a}}^{\widetilde{\phi}_{n}}$ in (4.1) as expected (in particular for $g=0)$.

\subsection{Outline of the paper}

Propositions 1.2 and 1.4 are straightforward to prove; this is done in Section 2.1. Explicit real orientations on the real-orientable complete intersections of Proposition 1.4 are described in Section 2.2 . We establish Theorem 1.6 and note additional properties of the real GW-invariants determined by these real orientations in Section 2.3. Theorem [1.5 is proved in Section 3. Section 4.1 introduces the equivariant setting relevant to the present situation. The resulting equivariant localization data is described in Section 4.2, see Theorem 4.6. Its use is illustrated in Section 4.3, Theorem 4.6 is proved in Section 5 , 


\section{Some examples}

This section establishes Propositions 1.2 and 1.4 and thus provides large collections of real-orientable symplectic manifolds. We also describe specific real orientations on the real-orientable symplectic manifolds of Propositions 1.4.

\subsection{Real orientable symplectic manifolds}

We begin by deducing Proposition 1.2 from topological observations made in [3].

Proof of Proposition 1.2. Let $(X, \omega, \phi)$ be a real symplectic manifold such that $w_{2}\left(X^{\phi}\right)=0$. We follow the proof of [3, Proposition 1.5].

Suppose $c_{1}(X)=2\left(\mu-\phi^{*} \mu\right)$ for some $\mu \in H^{2}(X ; \mathbb{Z})$. Let $L^{\prime} \longrightarrow X$ be a complex line bundle such that $c_{1}\left(L^{\prime}\right)=\mu$ and $(L, \widetilde{\phi})$ be the real bundle pair over $(X, \phi)$ given by

$$
L=L^{\prime} \otimes_{\mathbb{C}} \overline{\phi^{*} L^{\prime}}, \quad \widetilde{\phi}(v \otimes w)=w \otimes v .
$$

A continuous orientation on the fibers of $L^{\widetilde{\phi}} \longrightarrow X^{\phi}$ is given by the elements $v \otimes v$ with $\left.v \in L^{\prime}\right|_{X^{\phi}}$ nonzero. Thus, the first requirement in (1.3) is satisfied. Since $c_{1}(X)=2\left(\mu-\phi^{*} \mu\right)$, the complex line bundles $\Lambda_{\mathbb{C}}^{\text {top }} T X$ and $L^{\otimes 2}$ are isomorphic. If in addition $H_{1}(X ; \mathbb{Q})=0$, [3, Lemma 2.7] then implies that the second requirement in (1.3) is also satisfied.

Suppose $X$ is Kahler, $\phi$ is anti-holomorphic, and $\mathcal{K}_{X}=2\left([D]+\left[\overline{\phi_{*} D}\right]\right)$ for some divisor $D$ on $X$. Let $L^{\prime}=[-D]$ be the dual of the holomorphic line bundle corresponding to the divisor $D$ and $(L, \widetilde{\phi})$ be the real bundle pair over $(X, \phi)$ defined as in (2.1). The first requirement in (1.3) is again satisfied. In this case, $(L, \widetilde{\phi})$ is a holomorphic line bundle with an anti-holomorphic conjugation. Since $\mathcal{K}_{X}=2\left([D]+\left[\overline{\phi_{*} D}\right]\right)$, the holomorphic line bundles $\Lambda_{\mathbb{C}}^{\text {top }} T X$ and $L^{\otimes 2}$ are isomorphic. If in addition $X$ is compact, [3, Lemma 2.6] then implies that the second requirement in (1.3) is also satisfied.

Proof of Corollary 1.3. The assumptions imply that $X_{n ; \mathbf{a}} \subset \mathbb{P}^{n-1}$ is a threefold such that

$$
w_{1}\left(X_{\tau_{n ; \mathbf{a}}}^{\tau_{n ; \mathbf{a}}}\right)=0, \quad \mathcal{K}_{X_{n ; \mathbf{a}}}=2\left([D]+\left[\overline{\left\{\tau_{n ; \mathbf{a}}\right\}_{*} D}\right]\right) \text { with } D=\mathcal{O}_{\mathbb{P}^{n-1}}\left(\left(n-a_{1}-\ldots-a_{n-4}\right) / 4\right) .
$$

By Wu's relations [25, Theorem 11.14] and the first statement above, $w_{2}\left(X_{\tau_{n ; \mathbf{a}}}^{\tau_{n ; \mathbf{a}}}\right)=0$. The claim now follows from Proposition 1.2(2).

We now turn to the setting of Proposition 1.4. We will denote by $\mathfrak{c}$ the standard conjugation on $\mathbb{C}^{n}$. Define another conjugation on $\mathbb{C}^{n}$ by

$$
\mathfrak{c}_{\tau}^{\prime}\left(v_{1}, \ldots, v_{n}\right)= \begin{cases}\left(\bar{v}_{2}, \bar{v}_{1}, \ldots, \bar{v}_{n}, \bar{v}_{n-1}\right), & \text { if } n \in 2 \mathbb{Z} ; \\ \left(\bar{v}_{2}, \bar{v}_{1}, \ldots, \bar{v}_{n-1}, \bar{v}_{n-2}, \bar{v}_{n}\right), & \text { if } n \notin 2 \mathbb{Z} .\end{cases}
$$

We also define a $\mathbb{C}$-antilinear automorphism of $\mathbb{C}^{2 m}$ by

$$
\mathfrak{c}_{\eta}\left(v_{1}, \ldots, v_{2 m}\right)=\left(\bar{v}_{2},-\bar{v}_{1}, \ldots, \bar{v}_{2 m},-\bar{v}_{2 m-1}\right) .
$$

This automorphism has order 4 . 
For the purposes of equivariant localization computations, it is convenient to consider the involution

$$
\tau_{n}^{\prime}: \mathbb{P}^{n} \longrightarrow \mathbb{P}^{n}, \quad \tau_{n}^{\prime}\left(\left[Z_{1}, \ldots, Z_{n}\right]\right)=\left[\mathfrak{c}_{\tau}^{\prime}\left(Z_{1}, \ldots, Z_{n}\right)\right] .
$$

It is equivalent to the involution $\tau_{n}$ defined in Section 1.1 under the biholomorphic automorphism of $\mathbb{P}^{n}$ given by

$$
\left[Z_{1}, \ldots, Z_{2 m}\right] \longrightarrow \begin{cases}{\left[Z_{1}+\mathfrak{i} Z_{2}, Z_{1}-\mathfrak{i} Z_{2}, \ldots, Z_{n-1}+\mathfrak{i} Z_{n}, Z_{n-1}-\mathfrak{i} Z_{n}\right],} & \text { if } n \in 2 \mathbb{Z} \\ {\left[Z_{1}+\mathfrak{i} Z_{2}, Z_{1}-\mathfrak{i} Z_{2}, \ldots, Z_{n-2}+\mathfrak{i} Z_{n-1}, Z_{n-2}-\mathfrak{i} Z_{n-1}, Z_{n}\right],} & \text { if } n \notin 2 \mathbb{Z} .\end{cases}
$$

The involutions $\tau_{n}$ and $\tau_{n}^{\prime}$ lift to conjugations on the tautological line bundle

$$
\gamma_{n-1}=\mathcal{O}_{\mathbb{P}^{n-1}}(-1) \equiv\left\{(\ell, v) \in \mathbb{P}^{n-1} \times \mathbb{C}^{n}: v \in \ell \subset \mathbb{C}^{n}\right\}
$$

as

$$
\widetilde{\tau}_{n}(\ell, v)=\left(\tau_{n}(\ell), \mathfrak{c}(v)\right), \quad \widetilde{\tau}_{n}^{\prime}(\ell, v)=\left(\tau_{n}^{\prime}(\ell), \mathfrak{c}_{\tau}^{\prime}(v)\right) .
$$

For $a \in \mathbb{Z}$, we denote the induced conjugations on $\mathcal{O}_{\mathbb{P}^{n-1}}(a) \equiv\left(\gamma_{n-1}^{*}\right)^{\otimes a}$ by $\widetilde{\tau}_{n ; 1}^{(a)}$ and $\widetilde{\tau}_{n ; 1}^{(a)}$, respectively, omitting $(a)$ for $a=1$. The composition of $2 \widetilde{\tau}_{n ; 1}^{\prime(a)}$ with the involution

$$
2 \mathcal{O}_{\mathbb{P}^{n-1}}(a) \longrightarrow 2 \mathcal{O}_{\mathbb{P}^{n-1}}(a), \quad(x, y) \longrightarrow(y, x),
$$

is again an involution on $2 \mathcal{O}_{\mathbb{P}^{n-1}}(a)$; we denote it by $\widetilde{\tau}_{n ; 1,1}^{\prime(a)}$.

For $a \in \mathbb{Z}^{+}$, the involution $\phi=\eta_{2 m}$ lifts to a conjugation on $2 \gamma_{2 m-1}^{\otimes a}$ as

$$
\widetilde{\eta}_{2 m ; 1,1}^{(-a)}\left(\ell, v^{\otimes a}, w^{\otimes a}\right)=\left(\eta_{2 m}(\ell),\left(\mathfrak{c}_{\eta}(w)\right)^{\otimes a},\left(-\mathfrak{c}_{\eta}(v)\right)^{\otimes a}\right) .
$$

We denote the induced conjugations on

$$
2 \mathcal{O}_{\mathbb{P}^{2 m-1}}(a)=\left(2 \gamma_{2 m-1}^{\otimes a}\right)^{*} \quad \text { and } \quad \mathcal{O}_{\mathbb{P}^{2 m-1}}(2 a) \equiv \Lambda_{\mathbb{C}}^{2}\left(2 \mathcal{O}_{\mathbb{P}^{2 m-1}}(a)\right)
$$

by $\widetilde{\eta}_{2 m ; 1,1}^{(a)}$ and $\widetilde{\eta}_{2 m ; 1}^{(2 a)}$, respectively. We note that $\widetilde{\eta}_{2 m ; 1,1}^{(2 a)}=2 \widetilde{\eta}_{2 m ; 1}^{(2 a)}$.

For $\phi=\tau_{n}^{\prime}, \eta_{2 m}$, we define

$$
\phi:\{1, \ldots, n\} \longrightarrow\{1, \ldots, n\} \quad \text { by } \quad \phi(i)= \begin{cases}n, & \text { if } i=n \notin 2 \mathbb{Z} ; \\ 3 i-4\left\lfloor\frac{i}{2}\right\rfloor-1, & \text { otherwise }\end{cases}
$$

the second case interchanges each odd integer $2 k-1$ with its successor $2 k$. For $\phi=\tau_{n}$, we take the bijection in (2.5) to be the identity. Let

$$
|\phi|= \begin{cases}0, & \text { if } \phi=\tau_{n}, \tau_{n}^{\prime} \\ 1, & \text { if } \phi=\eta_{2 m}\end{cases}
$$


Lemma 2.1. Let $\phi$ denote either the involution $\tau_{n}$ or $\tau_{n}^{\prime}$ on $\mathbb{P}^{n-1}$ or the involution $\eta_{2 m}$ on $\mathbb{P}^{2 m-1}$. Euler's exact sequence of holomorphic vector bundles

$$
0 \longrightarrow \mathbb{P}^{n-1} \times \mathbb{C} \stackrel{f}{\longrightarrow} n \mathcal{O}_{\mathbb{P}^{n-1}}(1) \stackrel{h}{\longrightarrow} T \mathbb{P}^{n-1} \longrightarrow 0
$$

over $\mathbb{P}^{n-1}$ commutes with the conjugation $\phi \times \mathfrak{c}$ on the first term, the conjugation $\mathrm{d} \phi$ on the last term, and the conjugation $\tilde{\phi}_{n}^{*}$ on the middle term given by

- $n \widetilde{\tau}_{n ; 1}$ if $\phi=\tau_{n}$,

- $m \widetilde{\tau}_{n ; 1,1}^{\prime}$ if $\phi=\tau_{n}^{\prime}$ with $n=2 m$,

- $m \tilde{\tau}_{n ; 1,1}^{\prime} \oplus \tilde{\tau}_{n ; 1}^{\prime}$ if $\phi=\tau_{n}^{\prime}$ with $n=2 m+1$,

- $m \tilde{\eta}_{n ; 1,1}$ if $\phi=\eta_{n}$ with $n=2 m$.

Proof. For $i=1, \ldots, n$, define

$$
\begin{gathered}
\check{Z}_{i} \in H^{0}\left(\mathbb{P}^{n-1} ; \mathcal{O}_{\mathbb{P}^{n-1}}(1)\right) \quad \text { by } \quad\left\{\check{Z}_{i}(\ell)\right\}\left(\ell,\left(v_{1}, \ldots, v_{n}\right)\right)=v_{i} \forall\left(v_{1}, \ldots, v_{n}\right) \in \ell, \\
\mathbf{z}_{i}=\left(\operatorname{id},\left(z_{i 1}, \ldots, z_{i n}\right)\right): \mathcal{U}_{i} \equiv\left\{\left[Z_{1}, \ldots, Z_{n}\right] \in \mathbb{P}^{n-1}: Z_{i} \neq 0\right\} \longrightarrow \gamma_{n-1} \mid \mathcal{U}_{i}, \quad z_{i j}=\frac{Z_{j}}{Z_{i}}=\check{Z}_{j}\left(\mathbf{z}_{i}\right) .
\end{gathered}
$$

Thus, $\mathbf{z}_{i}$ is a section of $\gamma_{n-1}$ over $\mathcal{U}_{i},\left(z_{i j}\right)_{j \neq i}$ is a holomorphic chart on $\mathcal{U}_{i}$, and

$$
\frac{\partial}{\partial z_{i j}}=\sum_{j^{\prime} \neq i^{\prime}} \frac{\partial z_{i^{\prime} j^{\prime}}}{\partial z_{i j}} \frac{\partial}{\partial z_{i^{\prime} j^{\prime}}}= \begin{cases}z_{i i^{\prime}}^{-1} \frac{\partial}{\partial z_{i^{\prime} j}}, & \text { if } j \neq i^{\prime} \\ -z_{i i^{\prime}}^{-2}\left(\frac{\partial}{\partial z_{i^{\prime} i}}+\sum_{j^{\prime} \neq i, i^{\prime}} z_{i j^{\prime}} \frac{\partial}{\partial z_{i^{\prime} j^{\prime}}}\right), & \text { if } j=i^{\prime}\end{cases}
$$

on $\mathcal{U}_{i} \cap \mathcal{U}_{i^{\prime}}$ with $i \neq i^{\prime}$. The homomorphisms $f$ and $h$ in (2.7) are defined by

$$
\begin{gathered}
f(\ell, \lambda)=\left(\left.\lambda \check{Z}_{1}\right|_{\ell}, \ldots,\left.\lambda \check{Z}_{n}\right|_{\ell}\right) \quad \forall(\ell, \lambda) \in \mathbb{P}^{n-1} \times \mathbb{C}, \\
h\left(p_{1}, \ldots, p_{n}\right)=\left.\sum_{j \neq i}\left(p_{j}\left(\mathbf{z}_{i}(\ell)\right)-z_{i j}(\ell) p_{i}\left(\mathbf{z}_{i}(\ell)\right)\right) \frac{\partial}{\partial z_{i j}}\right|_{\ell} \quad \forall p_{1}, \ldots,\left.p_{n} \in \mathcal{O}_{\mathbb{P}^{n-1}}(1)\right|_{\ell}, \ell \in \mathcal{U}_{i} .
\end{gathered}
$$

It is straightforward to check that the last homomorphism is independent of the choice of $i$ and that the sequence (2.7) is indeed exact.

Denote by $\widetilde{\phi}$ the conjugation $\widetilde{\tau}_{n}$ if $\phi=\tau_{n}$, the conjugation $\widetilde{\tau}_{n}^{\prime}$ if $\phi=\tau_{n}^{\prime}$, and the real bundle automorphism

$$
\tilde{\phi}: \gamma_{2 m-1} \longrightarrow \gamma_{2 m-1}, \quad \tilde{\phi}(\ell, v)=\left(\eta_{2 m}(\ell), \mathfrak{c}_{\eta}(v)\right),
$$

if $\phi=\eta_{2 m}$; the square of (2.9) is - Id on each fiber. The effect of the involutions $\phi$ on the coordinate charts is described by the relations

$$
\check{Z}_{\phi(i)} \circ \widetilde{\phi}=(-1)^{|\phi| i} \mathfrak{c} \circ \check{Z}_{i}, \quad \mathbf{z}_{\phi(i)} \circ \phi=(-1)^{|\phi| i} \widetilde{\phi} \circ \mathbf{z}_{i} .
$$

Thus,

$$
z_{\phi(i) \phi(j)} \circ \phi=(-1)^{|\phi|(i+j)} \mathfrak{c} \circ z_{i j}, \quad \mathrm{~d}_{\ell} \phi\left(\left.\frac{\partial}{\partial z_{i j}}\right|_{\ell}\right)=\left.(-1)^{|\phi|(i+j)} \frac{\partial}{\partial z_{\phi(i) \phi(j)}}\right|_{\phi(\ell)} .
$$


Denote by $\widetilde{\phi}_{n}$ the conjugation on $\gamma_{n-1}$ dual to the conjugation $\widetilde{\phi}_{n}^{*}$ in the statement of the lemma. Thus,

$$
\left(\left\{\left(p_{1}, \ldots, p_{n}\right)\right\}\left(\tilde{\phi}_{n}\left(v_{1}, \ldots, v_{n}\right)\right)\right)_{\phi(i)}=(-1)^{|\phi| i} p_{\phi(i)}\left(\tilde{\phi}\left(v_{i}\right)\right) \begin{gathered}
\forall p_{1}, \ldots,\left.p_{n} \in \mathcal{O}_{\mathbb{P}^{n-1}}(1)\right|_{\ell}, \\
v_{1}, \ldots, v_{n} \in \ell .
\end{gathered}
$$

This identity is equivalent to

$$
\left(\left(p_{1}, \ldots, p_{n}\right) \circ \widetilde{\phi}_{n}\right)_{i}=(-1)^{|\phi| i} p_{\phi(i)} \circ \tilde{\phi} \quad \forall p_{1}, \ldots,\left.p_{n} \in \mathcal{O}_{\mathbb{P}^{n-1}}(1)\right|_{\ell}
$$

The first equation in (2.10) is equivalent to

$$
f(\phi(\ell), \bar{\lambda})=\mathfrak{c} \circ f(\ell, \lambda) \circ \widetilde{\phi}_{n}: n \phi(\ell) \longrightarrow \mathbb{C}^{n} \quad \forall(\ell, \lambda) \in \mathbb{P}^{n-1} \times \mathbb{C} .
$$

Since $\widetilde{\phi}^{2}=(-1)^{|\phi|} \mathrm{Id}$, the remaining equation in (2.10) and (2.11) are equivalent to

$$
h\left(\mathfrak{c} \circ\left(p_{1}, \ldots, p_{n}\right) \circ \widetilde{\phi}_{n}\right)=\mathrm{d}_{\ell} \phi\left(h\left(p_{1}, \ldots, p_{n}\right)\right) \quad \forall p_{1}, \ldots,\left.p_{n} \in \mathcal{O}_{\mathbb{P}^{n-1}}(1)\right|_{\ell}, \ell \in \mathbb{P}^{n} .
$$

The last two identities imply that $f$ and $h$ commute with the specified conjugations.

Let $\phi$ and $\widetilde{\phi}_{n}^{*}$ be as in Lemma 2.1 and define

$$
\widetilde{\phi}_{\mathcal{K}}= \begin{cases}\widetilde{\tau}_{n ; 1}^{(n)}, & \text { if } \phi=\tau_{n} \\ \widetilde{\tau}_{n ; 1}^{(n)}, & \text { if } \phi=\tau_{n}^{\prime} \\ \widetilde{\eta}_{2 m ; 1}^{(2 m)}, & \text { if } \phi=\eta_{2 m}\end{cases}
$$

The short exact sequence (2.7) induces an isomorphism

$$
\begin{aligned}
\Lambda_{\mathbb{C}}^{\text {top }}\left(T \mathbb{P}^{n-1}, \mathrm{~d} \phi\right) & =\Lambda_{\mathbb{C}}^{\text {top }}\left(\mathbb{P}^{n-1} \times \mathbb{C}, \phi \times \mathfrak{c}\right) \otimes \Lambda_{\mathbb{C}}^{\text {top }}\left(T \mathbb{P}^{n-1}, \mathrm{~d} \phi\right) \\
& \approx \Lambda_{\mathbb{C}}^{\text {top }}\left(n \mathcal{O}_{\mathbb{P}^{n-1}}(1), \widetilde{\phi}_{n}^{*}\right)=\left(\mathcal{O}_{\mathbb{P}^{n-1}}(n), \widetilde{\phi}_{\mathcal{K}}\right)
\end{aligned}
$$

of real bundle pairs over $\left(\mathbb{P}^{n-1}, \phi\right)$.

Proof of Proposition 1.4. Under the numerical assumptions in Proposition 1.4(1),

$$
\begin{aligned}
\left(\begin{array}{l}
n \\
2
\end{array}\right)-n \sum_{i=1}^{k} a_{i} & +\sum_{i=1}^{k} a_{i}^{2}+\sum_{i<j} a_{i} a_{j} \equiv\left(\begin{array}{l}
n \\
2
\end{array}\right)+0+\frac{1}{2}\left(|\mathbf{a}|^{2}-|\mathbf{a}|\right) \\
& \equiv \frac{1}{4}\left((n-|\mathbf{a}|)^{2}+(n+|\mathbf{a}|)(n+|\mathbf{a}|-2)\right) \equiv\left(\frac{n-|\mathbf{a}|}{2}\right)^{2} \bmod 2
\end{aligned}
$$

where $|\mathbf{a}|=a_{1}+\ldots+a_{k}$.

If $X_{n ; \mathbf{a}} \subset \mathbb{P}^{n-1}$ is a complete intersection preserved by $\tau_{n}$, the sequence

$$
\left.\left.0 \longrightarrow\left(T X_{n ; \mathbf{a}}, \mathrm{d} \tau_{n ; \mathbf{a}}\right) \longrightarrow\left(T \mathbb{P}^{n-1}, \mathrm{~d} \tau_{n}\right)\right|_{X_{n ; \mathbf{a}}} \longrightarrow \bigoplus_{i=1}^{k}\left(\mathcal{O}_{\mathbb{P}^{n-1}}\left(a_{i}\right), \widetilde{\tau}_{n ; 1}^{\left(a_{i}\right)}\right)\right|_{X_{n ; \mathbf{a}}} \longrightarrow 0
$$


is a short exact sequence of real bundle pairs over $\left(X_{n ; \mathbf{a}}, \tau_{n ; \mathbf{a}}\right)$. If $X_{2 m ; \mathbf{a}} \subset \mathbb{P}^{2 m-1}$ is a complete intersection preserved by $\eta_{2 m}$, the odd degrees $a_{i}$ come in pairs. There is thus a short exact sequence

$$
\begin{aligned}
0 \longrightarrow & \left.\left(T X_{2 m ; \mathbf{a}}, \mathrm{d} \eta_{2 m ; \mathbf{a}}\right) \longrightarrow\left(T \mathbb{P}^{2 m-1}, \mathrm{~d} \eta_{2 m}\right)\right|_{X_{2 m ; \mathbf{a}}} \\
\longrightarrow & \left.\left.\bigoplus{ }_{a_{i} \in 2 \mathbb{Z}}\left(\mathcal{O}_{\mathbb{P}^{2 m-1}}\left(a_{i}\right), \widetilde{\eta}_{2 m ; 1}^{\left(a_{i}\right)}\right)\right|_{X_{2 m ; \mathbf{a}}} \oplus \underset{a_{i}^{\prime} \notin 2 \mathbb{Z}}{\bigoplus}\left(\mathcal{O}_{\mathbb{P}^{2 m-1}}\left(a_{i}^{\prime}\right), \widetilde{\eta}_{2 m ; 1,1}^{\left(a_{i}^{\prime}\right)}\right)\right|_{X_{2 m ; \mathbf{a}}} \longrightarrow 0
\end{aligned}
$$

of real bundle pairs over $\left(X_{2 m ; \mathbf{a}}, \eta_{2 m ; \mathbf{a}}\right)$, where the second sum is taken over one $a_{i}^{\prime}=a_{i}$ for each odd-degree pair. The two exact sequences above determine isomorphisms

$$
\begin{aligned}
\Lambda_{\mathbb{C}}^{\mathrm{top}}\left(T X_{n ; \mathbf{a}}, \mathrm{d} \tau_{n ; \mathbf{a}}\right) \otimes\left(\mathcal{O}_{\mathbb{P}^{n-1}}(|\mathbf{a}|), \widetilde{\tau}_{n ; 1}^{(|\mathbf{a}|)}\right) & \left.\approx \Lambda_{\mathbb{C}}^{\mathrm{top}}\left(T \mathbb{P}^{n-1}, \mathrm{~d} \tau_{n}\right)\right|_{X_{n ; \mathbf{a}}}, \\
\Lambda_{\mathbb{C}}^{\mathrm{top}}\left(T X_{2 m ; \mathbf{a}}, \mathrm{d} \eta_{2 m ; \mathbf{a}}\right) \otimes\left(\mathcal{O}_{\mathbb{P}^{2 m-1}}(|\mathbf{a}|), \widetilde{\eta}_{2 m ; 1}^{(|\mathbf{a}|)}\right) & \left.\approx \Lambda_{\mathbb{C}}^{\mathrm{top}}\left(T \mathbb{P}^{2 m-1}, \mathrm{~d} \eta_{2 m}\right)\right|_{X_{2 m ; \mathbf{a}}}
\end{aligned}
$$

of real bundle pairs over $\left(X_{n ; \mathbf{a}}, \tau_{n ; \mathbf{a}}\right)$ and $\left(X_{2 m ; \mathbf{a}}, \eta_{2 m ; \mathbf{a}}\right)$, respectively.

Let $\nu_{n}(\mathbf{a})=n-|\mathbf{a}|$. By the assumptions of Proposition $1.4(1), \nu_{n}(\mathbf{a}) \in 2 \mathbb{Z}$. By the assumptions of Proposition $1.4(2), \nu_{2 m}(\mathbf{a}) \in 4 \mathbb{Z}$. Thus, the real bundle pairs

$$
\begin{aligned}
\left(L_{\tau ; n ; \mathbf{a}}, \widetilde{\phi}_{\tau ; n ; \mathbf{a}}\right) \equiv\left(\mathcal{O}_{\mathbb{P}^{n-1}}\left(\nu_{n}(\mathbf{a}) / 2\right), \widetilde{\tau}_{n ; 1}^{\left(\nu_{n}(\mathbf{a}) / 2\right)}\right) & \longrightarrow\left(\mathbb{P}^{n-1}, \tau_{n}\right), \\
\left(L_{\eta ; 2 m ; \mathbf{a}}, \widetilde{\phi}_{\eta ; 2 m ; \mathbf{a}}\right) \equiv\left(\mathcal{O}_{\mathbb{P}^{2 m-1}}\left(\nu_{2 m}(\mathbf{a}) / 2\right), \widetilde{\eta}_{2 m ; 1}^{\left(\nu_{2 m}(\mathbf{a}) / 2\right)}\right) & \longrightarrow\left(\mathbb{P}^{2 m-1}, \eta_{2 m}\right)
\end{aligned}
$$

are well-defined. By (2.12) and (2.16),

$$
\begin{aligned}
\Lambda_{\mathbb{C}}^{\mathrm{top}}\left(T X_{n ; \mathbf{a}}, \mathrm{d} \tau_{n ; \mathbf{a}}\right) & \approx\left(\left.\left(L_{\tau ; n ; \mathbf{a}}, \tilde{\phi}_{\tau ; n ; \mathbf{a}}\right)\right|_{X_{n ; \mathbf{a}}}\right)^{\otimes 2}, \\
\Lambda_{\mathbb{C}}^{\mathrm{top}}\left(T X_{2 m ; \mathbf{a}}, \mathrm{d} \eta_{2 m ; \mathbf{a}}\right) & \approx\left(\left.\left(L_{\eta ; 2 m ; \mathbf{a}}, \widetilde{\phi}_{\eta ; 2 m ; \mathbf{a}}\right)\right|_{X_{2 m ; \mathbf{a}}}\right)^{\otimes 2} .
\end{aligned}
$$

By (2.13),

$$
\begin{aligned}
w_{2}\left(X_{n ; \mathbf{a}}^{\tau_{n} ; \mathbf{a}}\right) & =\left(\left(\begin{array}{c}
n \\
2
\end{array}\right)-n \sum_{i=1}^{k} a_{i}+\sum_{i=1}^{k} a_{i}^{2}+\sum_{i<j} a_{i} a_{j}\right) x^{2} \\
& =\left(\frac{n-|\mathbf{a}|}{2}\right)^{2} x^{2}=w_{1}\left(L_{\tau ; n ; \mathbf{a}}^{\tilde{\phi}_{\tau ; n}}\right)^{2}
\end{aligned}
$$

where $x$ is the restriction of the generator of $H^{1}\left(\mathbb{R P}^{n-1} ; \mathbb{Z}_{2}\right)$ to $X_{n ; \mathbf{a}}^{\tau_{n ; \mathbf{a}}}$. Since $X_{2 m ; \mathbf{a}}^{\eta_{2 m}}=\varnothing$,

$$
w_{2}\left(X_{2 m ; \mathbf{a}}^{\eta_{2 m ; \mathbf{a}}}\right)=0=w_{1}\left(L_{\eta ; 2 m ; \mathbf{a}}^{\tilde{\phi}_{\eta ; 2 m ; \mathbf{a}}}\right)^{2} .
$$

Therefore, $\left(X_{n ; \mathbf{a}}, \omega_{n ; \mathbf{a}}, \tau_{n ; \mathbf{a}}\right)$ and $\left(X_{2 m ; \mathbf{a}}, \omega_{2 m ; \mathbf{a}}, \eta_{2 m ; \mathbf{a}}\right)$ are real-orientable symplectic manifolds under the assumptions in (1) and (2), respectively, of Proposition 1.4. 


\subsection{Real orientations on complete intersections}

We next describe a real orientation on each complete intersection $\left(X_{n ; \mathbf{a}}, \tau_{n ; \mathbf{a}}\right)$ and $\left(X_{2 m ; \mathbf{a}}, \eta_{2 m ; \mathbf{a}}\right)$ of Proposition 1.4 with

$$
(L, \widetilde{\phi})=\left(L_{\tau ; n ; \mathbf{a}}, \widetilde{\phi}_{\tau ; n ; \mathbf{a}}\right) \quad \text { and } \quad(L, \widetilde{\phi})=\left(L_{\eta ; 2 m ; \mathbf{a}}, \widetilde{\phi}_{\eta ; 2 m ; \mathbf{a}}\right) \text {, }
$$

respectively, as in (2.17). These real orientations are used to orient the normal bundles to torus fixed loci in Section 4 .

A homotopy class of isomorphisms as in (1.3) is determined by the corresponding isomorphism (2.18). By (2.19) and (2.20),$(L, \widetilde{\phi})$ satisfies the first condition in (1.3). Since $X_{2 m ; \mathbf{a}}^{\eta_{2 m}}=\varnothing$, this determines a real orientation on the complete intersections $\left(X_{2 m ; \mathbf{a}}, \eta_{2 m ; \mathbf{a}}\right)$ of Proposition 1.4(2).

In the case of the complete intersections $\left(X_{n ; \mathbf{a}}, \tau_{n ; \mathbf{a}}\right)$ of Proposition 1.4(1), it remains to specify a spin structure as in (RO3).

Lemma 2.2. Let $k \in \mathbb{Z}^{\geqslant 0}$ and $\mathbf{a} \equiv\left(a_{1}, \ldots, a_{k}\right) \in\left(\mathbb{Z}^{+}\right)^{k}$. If $\mathbf{a}$ satisfies the second condition in (1.5), then

$$
\left|\left\{i=1, \ldots, k: a_{i} \notin 2 \mathbb{Z}\right\}\right| \equiv \sum_{i=1}^{k} a_{i} \quad \bmod 4 .
$$

Proof. Since

$$
a_{i}^{2} \equiv\left\{\begin{array}{ll}
0, & \text { if } a_{i} \in 2 \mathbb{Z} ; \\
1, & \text { if } a_{i} \notin 2 \mathbb{Z} ;
\end{array} \quad \bmod 4,\right.
$$

the left-hand side of the second equation in (1.5) equals to the left-hand side of (2.21) modulo 4. This establishes the claim.

For each $a \in \mathbb{Z}$, let

$$
\mathcal{O}_{\mathbb{R P} \mathbb{P}^{n-1}}(a)=\mathcal{O}_{\mathbb{P}^{n-1}}(a)^{\widetilde{\tau}_{n ; 1}^{(a)}} \longrightarrow \mathbb{R P}^{n-1} .
$$

The canonical orientation on

$$
\mathcal{O}_{\mathbb{R P}^{n-1}}(2)=\mathcal{O}_{\mathbb{R P}^{n-1}}(1)^{\otimes 2} \longrightarrow \mathbb{R P}^{n-1}
$$

determines a homotopy class of isomorphisms

$$
\mathcal{O}_{\mathbb{R P}^{n-1}}(2 a) \approx \mathbb{R P}^{1} \times \mathbb{R} \quad \text { and } \quad \mathcal{O}_{\mathbb{R}^{n-1}}(2 a+1) \approx \mathcal{O}_{\mathbb{R P}^{n-1}}(1)
$$

of real line bundles over $\mathbb{R} \mathbb{P}^{n-1}$; the second isomorphism treats the last factor of $\mathcal{O}_{\mathbb{R}^{n-1}}(1)$ as the remainder.

Furthermore, the real vector bundle

$$
4 \mathcal{O}_{\mathbb{R} \mathbb{P}^{n-1}}(1) \longrightarrow \mathbb{R P}^{n-1}
$$

has a canonical orientation and spin structure. They are obtained by taking any trivialization of the first $2 \mathcal{O}_{\mathbb{R}^{p} n-1}(1)$ over a loop and taking the same trivialization over the last $2 \mathcal{O}_{\mathbb{R}^{n-1}}(1)$. These orientation and spin structure are invariant under the interchange of the first pair of the line 
bundles $\mathcal{O}_{\mathbb{R}^{n-1}}(1)$ with the last. The interchange of the components $\mathcal{O}_{\mathbb{R}^{n-1}}(1)$ within the same pair reverses the canonical orientation. By [12, Lemma 3.11], it also flips the spin structure (i.e. the homotopy classes of the canonical trivializations and their compositions with this interchange on the left do not differ by the compositions with an orientation-reversing diffeomorphism of $\mathbb{R}^{n}$ on the right).

We now return to the setting of Proposition 1.4(1). Let

$$
\ell_{0}(\mathbf{a})=\left|\left\{i=1, \ldots, k: a_{i} \in 2 \mathbb{Z}\right\}\right| \quad \text { and } \quad \ell_{1}(\mathbf{a})=\left|\left\{i=1, \ldots, k: a_{i} \notin 2 \mathbb{Z}\right\}\right|
$$

be the number of even entries in a and the number of odd entries, respectively. The short exact sequences (2.7) and (2.14) of real bundle pairs determine a homotopy class of isomorphisms

$$
\begin{aligned}
\left(X_{n ; \mathbf{a}}^{\tau_{n} \mathbf{a}} \times \mathbb{R}\right) \oplus\left(\left.T X_{n ; \mathbf{a}}^{\tau_{n} ; \mathbf{a}} \oplus 2\left(L^{*}\right)^{\tilde{\phi}^{*}}\right|_{X_{n ; \mathbf{a}}^{\tau_{n} ; \mathbf{a}}}\right) & \left.\oplus \bigoplus_{i=1}^{k} \mathcal{O}_{\mathbb{R P P}^{n-1}}\left(a_{i}\right)\right|_{X_{n ; \mathbf{a}}^{\tau_{n}}} \\
& \left.\left.\approx n \mathcal{O}_{\mathbb{R P}^{n-1}}(1)\right|_{X_{n ; \mathbf{a}}^{\tau_{n}}} \oplus 2\left(L^{*}\right)^{\tilde{\phi}^{*}}\right|_{X_{n ; \mathbf{a}}^{\tau_{n}}}
\end{aligned}
$$

of real vector bundles over $X_{n ; \mathbf{a}}^{\tau_{n} \text {. }}$. If $n-|\mathbf{a}| \in 4 \mathbb{Z},(2.22)$ and (2.23) determine a homotopy class of isomorphisms

$$
\begin{aligned}
& \left.\left(X_{n ; \mathbf{a}}^{\tau_{n} ; \mathbf{a}} \times \mathbb{R}\right) \oplus\left(\left.T X_{n ; \mathbf{a}}^{\tau_{n} ; \mathbf{a}} \oplus 2\left(L^{*}\right)^{\tilde{\phi}^{*}}\right|_{X_{n ; \mathbf{a}}^{\tau_{n} ; \mathbf{a}}}\right) \oplus\left(X_{n ; \mathbf{a}}^{\tau_{n} ; \mathbf{a}} \times \mathbb{R}^{\ell_{0}(\mathbf{a})}\right) \oplus \ell_{1}(\mathbf{a}) \mathcal{O}_{\mathbb{R}^{n-1}}(1)\right|_{X_{n ; \mathbf{a}}^{\tau_{n} ; \mathbf{a}}} \\
& \left.\left.\approx\left(n-\ell_{1}(\mathbf{a})\right) \mathcal{O}_{\mathbb{R P}^{n-1}}(1)\right|_{X_{n ; \mathbf{a}}^{\tau_{n} ; \mathbf{a}}} \oplus \ell_{1}(\mathbf{a}) \mathcal{O}_{\mathbb{R P}^{n-1}}(1)\right|_{X_{n ; \mathbf{a}}^{\tau_{n} ; \mathbf{a}}} \oplus\left(X_{n ; \mathbf{a}}^{\tau_{n ; \mathbf{a}}} \times \mathbb{R}^{2}\right) .
\end{aligned}
$$

If $n-|\mathbf{a}| \notin 4 \mathbb{Z},(2.22)$ and (2.23) determine a homotopy class of isomorphisms

$$
\begin{aligned}
\left.\left(X_{n ; \mathbf{a}}^{\tau_{n} ; \mathbf{a}} \times \mathbb{R}\right) \oplus\left(\left.T X_{n ; \mathbf{a}}^{\tau_{n} ; \mathbf{a}} \oplus 2\left(L^{*}\right)^{\tilde{\phi}^{*}}\right|_{X_{n ; \mathbf{a}}^{\tau_{n} ; \mathbf{a}}}\right) \oplus\left(X_{n ; \mathbf{a}}^{\tau_{n} ; \mathbf{a}} \times \mathbb{R}^{\ell_{0}(\mathbf{a})}\right) \oplus \ell_{1}(\mathbf{a}) \mathcal{O}_{\mathbb{R} \mathbb{P}^{n-1}}(1)\right|_{X_{n ; \mathbf{a}}^{\tau_{n}}} \\
\left.\left.\approx\left(n+2-\ell_{1}(\mathbf{a})\right) \mathcal{O}_{\mathbb{R}^{n-1}}(1)\right|_{X_{n ; \mathbf{a}}^{\tau_{n} \mathbf{a}}} \oplus \ell_{1}(\mathbf{a}) \mathcal{O}_{\mathbb{R}^{n-1}}(1)\right|_{X_{n ; \mathbf{a}}^{\tau_{n} ; \mathbf{a}}}
\end{aligned}
$$

The first terms on the right-hand sides of (2.24) and (2.25) correspond to the first $n-\ell_{1}(\mathbf{a})$ and $n+2-\ell_{1}(\mathbf{a})$ summands of the first term on the right-hand sides of (2.23).

By Lemma 2.2, the ranks of the first summands on the right-hand sides of (2.24) and (2.25) are divisible by 4 . Thus, (2.24) and (2.25) determine a homotopy class of isomorphisms

$$
\begin{aligned}
\left(X_{n ; \mathbf{a}}^{\tau_{n} ; \mathbf{a}} \times \mathbb{R}\right) \oplus\left(\left.T X_{n ; \mathbf{a}}^{\tau_{n} ; \mathbf{a}} \oplus 2\left(L^{*}\right)^{*}\right|_{X_{n ; \mathbf{a}}^{\tau_{n} ; \mathbf{a}}}\right) & \left.\oplus\left(X_{n ; \mathbf{a}}^{\tau_{n} ; \mathbf{a}} \times \mathbb{R}^{\ell_{0}(\mathbf{a})}\right) \oplus \ell_{1}(\mathbf{a}) \mathcal{O}_{\mathbb{R} \mathbb{P}^{n-1}}(1)\right|_{X_{n ; \mathbf{a}}^{\tau_{n} ; \mathbf{a}}} \\
& \left.\approx\left(X_{n ; \mathbf{a}}^{\tau_{n} \mathbf{a}} \times \mathbb{R}^{n+2-\ell_{1}(\mathbf{a})}\right) \oplus \ell_{1}(\mathbf{a}) \mathcal{O}_{\mathbb{R}^{\mathbb{P}^{n-1}}}(1)\right|_{X_{n ; \mathbf{a}}^{\tau_{n} ; \mathbf{a}}}
\end{aligned}
$$

over every loop in $X_{n ; \mathbf{a}}^{\tau_{n}}$. Since the real bundle pair $(L, \widetilde{\phi})$ satisfies (1.3), the real orientable vector bundle $T X_{n ; \mathbf{a}}^{\tau_{n} \mathbf{a}} \oplus 2\left(L^{*}\right)^{\tilde{\phi}^{*}}$ admits a spin structure. By the first two statements of [12, Lemma 3.11], it is determined by (2.26) and the orientation on $T X^{\phi}$ specified by the first isomorphism in (2.18).

\subsection{The canonical orientations of the moduli spaces}

By [11, Theorem 1.3], the real orientations on $\left(X_{n ; \mathbf{a}}, \tau_{n ; \mathbf{a}}\right)$ and $\left(X_{2 m ; \mathbf{a}}, \eta_{2 m ; \mathbf{a}}\right)$ constructed in Section 2.2 induce orientations on the moduli spaces of real maps into these real symplectic manifolds 
if $n-k \in 2 \mathbb{Z}$ (so that the complex dimensions of these Kahler manifolds are odd). The construction of these real orientations involves some implicit choices (which are listed explicitly in the statement of Theorem 1.6). Below we describe the effect of these choices on the real orientations, the induced orientations on the moduli spaces of real maps, and the resulting GW-invariants of $\left(X_{n ; \mathbf{a}}, \tau_{n ; \mathbf{a}}\right)$ and $\left(X_{2 m ; \mathbf{a}}, \eta_{2 m ; \mathbf{a}}\right)$. We use this description to establish Theorem 1.6. Proposition 2.6 notes additional properties of the real orientations of Section 2.2.

Throughout this section, we denote by $(X, \phi)$ either a fixed complete intersection $\left(X_{n ; \mathbf{a}}, \tau_{n ; \mathbf{a}}\right)$ in $\left(\mathbb{P}^{n-1}, \tau_{n}\right)$ cut out by a real holomorphic bundle section $s_{n ; \mathbf{a}}$ or a fixed complete intersection $\left(X_{2 m ; \mathbf{a}}, \eta_{2 m ; \mathbf{a}}\right)$ in $\left(\mathbb{P}^{2 m-1}, \eta_{2 m}\right)$ cut out by a real holomorphic bundle section $s_{2 m ; \mathbf{a}}$. Two such sections cut out the same complete intersections if and only if they differ by a real holomorphic automorphism of the holomorphic vector bundle

$$
\mathcal{L}_{n ; \mathbf{a}} \equiv \bigoplus_{i=1}^{k} \mathcal{O}_{\mathbb{P}^{n-1}}\left(a_{i}\right) \longrightarrow \mathbb{P}^{n-1}
$$

A holomorphic endomorphism $\Phi$ of (2.27) corresponds to $k^{2}$ elements

$$
\varphi_{i j} \in H^{0}\left(\mathbb{P}^{n-1} ; \mathcal{O}_{\mathbb{P}^{n-1}}\left(a_{i}-a_{j}\right)\right), \quad i, j=1, \ldots, k .
$$

In particular, $\varphi_{i j}=0$ if $a_{i}<a_{j}$ and $\varphi_{i j} \in \mathbb{C}$ if $a_{i}=a_{j}$. This endomorphism is thus invertible if and only if the (constant) matrix $\left(\varphi_{i j}^{\prime}\right)_{i j}$ given by

$$
\varphi_{i j}^{\prime}= \begin{cases}\varphi_{i j}, & \text { if } a_{i}=a_{j} \\ 0, & \text { if } a_{i} \neq a_{j}\end{cases}
$$

is invertible. If $\phi=\tau_{n ; \mathbf{a}}, \Phi$ is real if each $\varphi_{i j}$ in (2.28) is real. The homotopy classes of real automorphisms of (2.27) in this case are thus generated by negating the individual components of (2.27). If $\phi=\eta_{2 m ; \mathbf{a}}$, the odd-degree line bundles in (2.28) are paired up. Every real holomorphic automorphism of the associated rank 2 real bundle pair is homotopic to the identity through real holomorphic automorphisms. The homotopy classes of real automorphisms of (2.27) in this case are thus generated by negating the individual even-degree components of (2.27).

Remark 2.3. The real bundle pairs $\left(2 \mathcal{O}_{\mathbb{P}^{2 m-1}}(a), \widetilde{\eta}_{2 m ; 1,1}^{(a)}\right)$ with $a \notin 2 \mathbb{Z}$ admit continuous real automorphisms not homotopic to the identity. Their homotopy classes are characterized by their restrictions to an equator $S^{1} \subset \mathbb{C}^{*}$ inside of a real linearly embedded $\mathbb{P}^{1} \subset \mathbb{P}^{n-1}$ being homotopic to the automorphism

$$
(\alpha, \beta) \longrightarrow\left(z^{-1} \beta, z \alpha\right)
$$

This homomorphism extends continuously, but not holomorphically, over $\mathbb{P}^{n-1}$.

Whenever the choices implicitly made in Section 2.2 affect the resulting real orientation on $\left(X_{n ; \mathbf{a}}, \phi\right)$ constructed in Section 2.2, their effects on the induced orientations of the moduli spaces of real maps are straightforward to determine using oriented symmetric half-surfaces (or sh-surfaces) as in [10] and [12, Section 3.2]. An sh-surface $\left(\Sigma^{b}, c\right)$ contains ordinary boundary components and crosscaps (boundary components with an antipodal involution) and doubles to a symmetric surface $(\Sigma, \sigma)$. The parity of the total number of the boundary components of $\Sigma^{b}$ is the parity of $g+1$, 
where $g$ is the genus of $\Sigma$.

The first choice made in Section 2.2 is a real holomorphic parametrization of $\left(\mathbb{P}^{n-1}, \tau_{n}\right)$ and $\left(\mathbb{P}^{2 m-1}, \eta_{2 m}\right)$; this in particular includes the ordering of the homogeneous components for the purposes of Lemma 2.1. Since the group $\operatorname{Aut}\left(\mathbb{P}^{1}, \eta\right)$ is connected, the group $\operatorname{Aut}\left(\mathbb{P}^{2 m-1}, \eta_{2 m}\right)$ is also connected. A path in $\operatorname{Aut}\left(\mathbb{P}^{2 m-1}, \eta_{2 m}\right)$ lifts to a path of holomorphic automorphisms of $m\left(2 \mathcal{O}_{\mathbb{P}^{2 m-1}}(1), \widetilde{\eta}_{2 m ; 1,1}^{(1)}\right)$. Since the group of holomorphic automorphisms of this real bundle pair over the identity is connected, it follows that the real orientation of Section 2.2 is independent of the choice of real holomorphic parametrization of $\left(\mathbb{P}^{2 m-1}, \eta_{2 m}\right)$.

The group $\operatorname{Aut}\left(\mathbb{P}^{1}, \tau\right)$ has two connected components; the negation of a homogenous coordinate is not homotopic to the identity. Since such an automorphism reverses the orientation of $\mathbb{R P}^{n-1}$ whenever $n \in 2 \mathbb{Z}^{+}$, this implies that $\operatorname{Aut}\left(\mathbb{P}^{n-1}, \tau_{n}\right)$ has precisely two connected components for any $n \geqslant 2$. Negating a homogenous coordinate of $\mathbb{P}^{n}$ changes

(C0) the homotopy class of the surjection $h$ in (2.7),

(C1) the homotopy class of the isomorphism in (1.3) induced by the first isomorphism in (2.18) over every real loop in $X_{n ; \mathbf{a}}$, and

(C2) the orientation on $T X_{n ; \mathbf{a}}^{\tau_{n ; \mathbf{a}}} \oplus 2\left(L^{*}\right)^{\tilde{\phi}^{*}}$ induced by the isomorphism (2.26).

By the third statement of [12, Lemma 3.11], such an interchange also changes

(C3) the resulting spin of $T X_{n ; \mathbf{a}}^{\tau_{n} \mathbf{a}} \oplus 2\left(L^{*}\right)^{\tilde{\phi}^{*}}$ over every loop in $X_{n ; \mathbf{a}}^{\tau_{n} \mathbf{a}}$ not contractible in $\mathbb{R P P}^{n-1}$.

For a real map $u$ from an sh-surface $\Sigma^{b}$, the effects of these changes on the trivializations over the boundary components of $\Sigma^{b}$ are to flip

(E1) the homotopy type of trivializations of $u^{*}\left(T X_{n ; \mathbf{a}}, \mathrm{d} \tau_{n ; \mathbf{a}}\right)$ over each crosscap,

(E2) the orientation of trivializations of $u^{*} T X_{n ; \mathbf{a}}^{\tau_{n ;}}$ over each ordinary boundary component,

(E3) the spin of trivializations of $u^{*} T X_{n ; \mathbf{a}}^{\tau_{n} ; \mathbf{a}}$ over each ordinary boundary component to which the restriction of $u$ is homotopically non-trivial as a map to $\mathbb{R} \mathbb{P}^{n-1}$.

By [2, Propositions 4.1,4.2], the parity of the number of the components in (E3) is the parity of the degree $d$ of $u$.

Proof of Theorem 1.6. Let $\widetilde{\eta}_{2 m}$ be an anti-holomorphic conjugation on the vector bundle $\mathcal{L}_{n ; \mathbf{a}}$ in (2.27) lifting the involution $\eta_{2 m}$ on $\mathbb{P}^{2 m-1}$ and

$$
H^{0}\left(\mathbb{P}^{2 m-1} ; \mathcal{L}_{n ; \mathbf{a}}\right)^{\widetilde{\eta}_{2 m}} \subset H^{0}\left(\mathbb{P}^{2 m-1} ; \mathcal{L}_{n ; \mathbf{a}}\right)
$$

be the subspace of real holomorphic sections. Since the fixed locus of the involution $\eta_{2 m}$ on $\mathbb{P}^{2 m-1}$ is empty, the subspace

$$
\left\{(s, p) \in H^{0}\left(\mathbb{P}^{2 m-1} ; \mathcal{L}_{n ; \mathbf{a}}\right)^{\widetilde{\eta}_{2 m}} \times \mathbb{P}^{n-1}: s(p)=0,\left.\operatorname{rk}_{\mathbb{C}} \nabla s\right|_{p}<k\right\}
$$

has complex codimension $n$ in $H^{0}\left(\mathbb{P}^{2 m-1} ; \mathcal{L}_{n ; \mathbf{a}}\right)^{\widetilde{\eta}_{2 m}} \times \mathbb{P}^{n-1}$. Thus, its projection to the first component has complex codimension one and the space of regular real sections of (2.27) is path-connected. 
Along with the conclusions above Remark 2.3 and in the paragraph concerning $\operatorname{Aut}\left(\mathbb{P}^{2 m-1}, \eta_{2 m}\right)$, this implies that the real orientation on $\left(X_{2 m ; \mathbf{a}}, \eta_{2 m ; \mathbf{a}}\right)$ constructed in Section 2.2 and the resulting real GW-invariants are independent of the real parametrization of $\left(\mathbb{P}^{2 m-1}, \eta_{2 m}\right)$, the ordering of the line bundles associated with the complete intersection, and the section $s_{2 m ; \mathbf{a}}$.

The first vanishing claim of Theorem 1.6](2) in this case follows from Lemma 2.5 below (its $g=0$ case is contained in [3, Lemma 1.9]). If $a_{i} \in 2 \mathbb{Z}$ for some $i$, replacing the component $s_{i}$ of $s_{n ; \mathbf{a}}$ by $-s_{i}$ results in the changes in (C1) and thus in (E1). If $g \in 2 \mathbb{Z}$, this replacement thus changes the orientation of the moduli space of real maps to $\left(X_{2 m ; \mathbf{a}}, \eta_{2 m ; \mathbf{a}}\right)$ and the sign of the corresponding real GW-invariants. However, by the previous paragraph, these invariants are independent of the choice of the section $s_{n ; \mathbf{a}}$. This establishes the second vanishing claim of Theorem 1.6](2) for $\phi=\eta_{2 m ; \mathbf{a}}$.

We next consider the case $\phi=\tau_{n ; \mathbf{a}}$. A real holomorphic reparametrization of $\left(\mathbb{P}^{n-1}, \tau_{n}\right)$ induced by a linear automorphism $\varphi$ of $\mathbb{C}^{n}$ determines a commutative diagram of the form

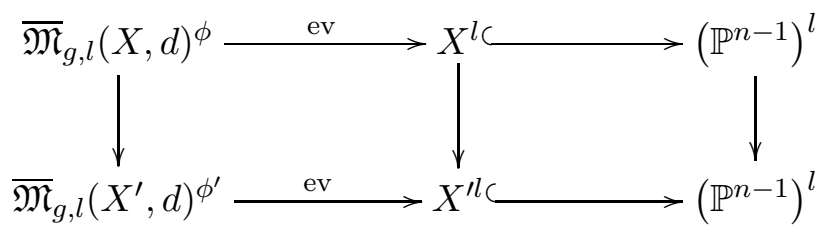

with $\left(X^{\prime}, \phi^{\prime}\right)$ denoting the complete intersection cut out by the section $s_{n ; \mathbf{a}}^{\prime}$ obtained from $s_{n ; \mathbf{a}}$ by a suitable transform. The middle and right vertical arrows are orientation-preserving with respect to the canonical complex orientations on their domains and targets. The left vertical arrow is orientation-preserving with respect to the orientations of Section 2.2 differing by the reparametrization of the middle term in (2.7) by $\varphi$. The GW-invariants of Theorem 1.6 are the intersection numbers of the first horizontal arrows with the cycles represented by the constraints.

By the commutativity of the first square in (2.29), the GW-invariants of Theorem 1.6 are thus invariant under real holomorphic reparametrizations of $\left(\mathbb{P}^{n-1}, \tau_{n}\right)$. This in particular establishes Theorem 1.6](1) for $k=0$. Negating one of the $\mathcal{O}_{\mathbb{P}^{n-1}}$ (1)-factors in (2.7) or one of the odd-degree components $s_{i}$ of $s_{n ; \mathbf{a}}$ affects (C1) (C3) and the orientation of $\overline{\mathfrak{M}}_{g, l}(X, d)^{\phi}$ through (E1) (E3), Each of the last three changes by itself would reverse the orientation of $\overline{\mathfrak{M}}_{g, l}(X, d)^{\phi}$. Since the parity of the number of changes (E1) and (E2) is that of $g+1$ and of (E3) is that of $d$, negating one of the $\mathcal{O}_{\mathbb{P}^{n-1}}(1)$-factors in (2.7) or one of the odd-degree components $s_{i}$ of $s_{n ; \mathbf{a}}$ preserves the orientation of $\overline{\mathfrak{M}}_{g, l}(X, d)^{\phi}$ if $d-g \notin 2 \mathbb{Z}$ and reverses it otherwise. This establishes Theorem 1.6](1) under the assumptions that $a_{i} \notin 2 \mathbb{Z}$ for all $i$ and $d-g \notin 2 \mathbb{Z}$. Since negating an even-degree component $s_{i}$ of $s_{n ; \mathbf{a}}$ affects (C1) and (C2) only and the orientation of $\overline{\mathfrak{M}}_{g, l}(X, d)^{\phi}$ through (E1) and (E2), this operation preserves the orientation of $\overline{\mathfrak{M}}_{g, l}(X, d)^{\phi}$ if $g \notin 2 \mathbb{Z}$ and reverses it otherwise. Combined with the previous observation, this establishes Theorem 1.6.(1) under the assumption $g \notin 2 \mathbb{Z}$ for all $i$ and $d \in 2 \mathbb{Z}$.

We now turn to Theorem 1.6] with $\phi=\tau_{n ; \mathbf{a}}$. If $d-g \in 2 \mathbb{Z}$, negating one of the $\mathcal{O}_{\mathbb{P}^{n-1}}(1)$-factors in (2.7) changes the orientation of $\overline{\mathfrak{M}}_{g, l}(X, d)^{\phi}$ and thus the sign of the real GW-invariants of $(X, \phi)$. If $a_{i} \in 2 \mathbb{Z}$ for some $i$ and $g \in 2 \mathbb{Z}$, replacing $s_{i}$ by $-s_{i}$ changes the sign of the real GW-invariants of $(X, \phi)$. If the real genus $g$ degree $d \mathrm{GW}$-invariants of $(X, \phi)$ are invariant under these changes, 
then they must vanish.

It remains to consider the $g=0$ case of Theorem 1.6.(1). The real GW-invariants of $(X, \phi)$ are then given by cupping the constraints with the Euler class of the bundle (4.1) to $\overline{\mathfrak{M}}_{g, l}\left(\mathbb{P}^{n-1}, d\right)^{\tau_{n}}$. The proof of [28, Theorem 3] establishes this statement for $(n, \mathbf{a})=(5,(5))$ and $d \notin 2 \mathbb{Z}$, but its principles apply in general (as long as $g=0$ ). The real GW-invariants of $(X, \phi)$ can then be computed using the equivariant localization theorem of [1] as in Section 4.2 . If $d \in 2 \mathbb{Z}$ (i.e. $d-g \in 2 \mathbb{Z}$ ) or $a_{i} \in 2 \mathbb{Z}$ for some $i$, then all torus fixed loci contribute zero to these invariants; see Lemma 5.3. Therefore, the $g=0$ real $\mathrm{GW}$-invariants vanish in these cases and are in particular independent of all choices implicitly made in Section 2.2. The same reasoning applies whenever the real genus $g$ degree $d$ GW-invariants can be similarly related to $\overline{\mathfrak{M}}_{g, l}\left(\mathbb{P}^{n-1}, d\right)^{\tau_{n}}$ and either $d-g \in 2 \mathbb{Z}$ or $a_{i} \in 2 \mathbb{Z}$ for some $i$ and $g \in 2 \mathbb{Z}$.

Remark 2.4. The independence of the real GW-invariants of $(X, \phi)$ in the $\phi=\eta_{n ; \mathbf{a}}$ case is established by taking the homotopy between two moduli spaces of real maps induced by a generic path between two regular real sections of (2.27); it consists of regular sections in this case. Such a path need not exist in the $\phi=\tau_{n ; \mathbf{a}}$ case, but a cobordism between the moduli space would satisfy. It would pass through spaces of real maps into hypersurfaces with isolated real nodal points. Unfortunately, we do not see at this point a notion of a moduli space which would be suitable for constructing the desired cobordism.

Lemma 2.5. Suppose $m, d \in \mathbb{Z}^{+}$and $g, l \in \mathbb{Z}^{\geqslant 0}$. If $d-g \in 2 \mathbb{Z}$, then

$$
\overline{\mathfrak{M}}_{g, l}\left(\mathbb{P}^{2 m-1}, d\right)^{\eta_{2 m}}=\varnothing .
$$

Proof. It is sufficient to establish this statement for $l=0$ and $m=1$ (after composition with a projection to a generic real line). By the Riemann-Hurwitz formula [16, p219], a genus $g$ degree $d$ cover of $\mathbb{P}^{1}$ has $2(d+g-1)$ branched points in $\mathbb{P}^{1}$, counted with multiplicity. Any such cover is determined by the branched points and some combinatorial data. Suppose some combinatorial data is compatible with the involution $\eta_{2}$ on $\mathbb{P}^{1}$ and some (necessarily fixed-point-free) involution on the domain. We take the limit of such real covers by bringing all of the branched points to a pair of conjugate points. The restriction of the limiting map to each non-contracted component of the domain then has no branched points; any such component is thus a $\mathbb{P}^{1}$ and the degree of the limiting map is 1 . Denote by $g_{0}$ the sum of the geometric genera of the contracted components and by $N$ the number of nodes of the domain of the limiting map. Thus,

$$
g=d-N+1+g_{0} .
$$

Since the fixed locus of $\left(\mathbb{P}^{1}, \eta\right)$ is empty, $g_{0}, N \in 2 \mathbb{Z}$. The claim thus follows from (2.30).

Since the involutions $\tau_{n}$ and $\tau_{n}^{\prime}$ on $\mathbb{P}^{n-1}$ are related by the automorphism (2.3), the construction of real orientations in Section 2.2 applies with only minor changes to complete intersections invariant under the involutions $\tau_{n}^{\prime}$. For such complete intersections, some of the odd-degree sections $s_{i}$ may be paired up (the sections corresponding to the real bundle pairs $\left(2 \mathcal{O}_{\mathbb{P}^{n-1}}(a), \widetilde{\tau}_{n ; 1,1}^{\prime(a)}\right)$ ). However, the ordering of the sections within each such pair is not fixed (because the automorphism $\tilde{\phi}$ in the proof of Lemma 2.1 is of order 2 in this case). The same invariance considerations as in the $\tau_{n}$ case apply with the involution $\tau_{n}^{\prime}$.

We next note some properties of the real orientations constructed in Section 2.2 . 
Proposition 2.6. (1) Let $m, n \in \mathbb{Z}^{+}, k \in \mathbb{Z}^{\geqslant 0}$, and $\mathbf{a} \equiv\left(a_{1}, \ldots, a_{k}\right) \in\left(\mathbb{Z}^{+}\right)^{k}$. If the real orientations on $\left(X_{n ; \mathbf{a}}, \tau_{n ; \mathbf{a}}\right)$ and $\left(X_{2 m ; \mathbf{a}}, \eta_{2 m ; \mathbf{a}}\right)$ constructed in Section 2.2 are independent of the choices made, then they are also invariant under the inclusions

$$
\left(\mathbb{P}^{n-1}, \tau_{n}\right) \longrightarrow\left(\mathbb{P}^{n}, \tau_{n+1}\right) \quad \text { and }\left(\mathbb{P}^{2 m-1}, \eta_{2 m}\right) \longrightarrow\left(\mathbb{P}^{2 m+1}, \eta_{2 m+2}\right)
$$

as coordinate subspaces. If the induced orientation on the moduli space of genus $g$ degree $d$ real maps is independent of these choices, then it is also invariant under the inclusions (2.31).

(2) Let $m \in \mathbb{Z}^{+}$. The signed count of real lines through a pair of conjugate points in $\left(\mathbb{P}^{2 m-1}, \tau_{2 m}\right)$ with respect to the real orientation of Section 2.2 is given by (1.1).

Proof. (1) The first inclusion in (2.31) replaces $X_{n ; \mathbf{a}}$ with $X_{n+1 ; \mathbf{a}^{\prime}}$, where $\mathbf{a}^{\prime}$ is tuple obtained from $\mathbf{a}$ by adding 1 as the last component. The only effect of this change on (2.24)-(2.26) is to increase the coefficients $\ell_{1}(\mathbf{a})$ in front of $\left.\mathcal{O}_{\mathbb{R}^{n-1}}(1)\right|_{X_{n ; \mathbf{a}}^{\tau}} ^{\tau_{n} ; \mathbf{a}}$ by 1 . There is no effect on the homotopy class of the isomorphism in (1.3) induced by the first isomorphism in (2.18) or on the spin structure on $T X_{n ; \mathbf{a}}^{\tau_{n} \mathbf{a}} \oplus 2\left(L^{*}\right)^{\tilde{\phi}^{*}}$.

The second inclusion in (2.31) replaces $X_{2 m ; \mathbf{a}}$ with $X_{2 m+2 ; \mathbf{a}^{\prime}}$, where $\mathbf{a}^{\prime}$ is tuple obtained from a by adding 1 as the last two components. There is no effect on the homotopy class of the isomorphism in (1.3) induced by the second isomorphism in (2.18).

The second inclusion in (2.31) replaces $X_{2 m ; \mathbf{a}}$ with $X_{2 m+2 ; \mathbf{a}^{\prime}}$, where $\mathbf{a}^{\prime}$ is tuple obtained from a by adding 1 as the last two components. There is no effect on the homotopy class of the isomorphism in (1.3) induced by the second isomorphism in (2.18).

(2) Suppose first $m \in 2 \mathbb{Z}$. The real part of the first line bundle in (2.17) is then orientable. By [12, Theorem 1.5], the orientation on

$$
\overline{\mathfrak{M}}_{0,1}\left(\mathbb{P}^{2 m-1}, 1\right)^{\tau_{2 m}}=\mathfrak{M}_{0,1}\left(\mathbb{P}^{2 m-1}, 1\right)^{\tau_{2 m}}
$$

induced by the real orientation of Section 2.2 is the orientation induced by the associated spin structure on $T \mathbb{R P}^{2 m-1}$. The latter is induced by the canonical spin structure on $2 m \mathcal{O}_{\mathbb{R}^{2 m-1}}(1)$ via Euler's sequence as in [3, Section 5.5]. The claim now follows from [3, (1.21)].

Suppose now $m \notin 2 \mathbb{Z}$. The real part of the first line bundle in (2.17) is then non-orientable. By [12, Theorem 1.5], the orientation on (2.32) induced by the real orientation of Section 2.2 agrees with the orientation induced by the associated relative spin structure if and only if $m+1 \in 4 \mathbb{Z}$. This is the relative spin structure in the sense of [6, Theorem 8.1.1] associated with the oriented rank 2 real vector bundle

$$
\mathcal{O}_{\mathbb{P}^{2 m-1}}(-m) \longrightarrow \mathbb{P}^{2 m-1}
$$

and the canonical spin structure on

$$
\begin{aligned}
\left.2 m \mathcal{O}_{\mathbb{R P}^{2 m-1}}(1) \oplus \mathcal{O}_{\mathbb{P}^{2 m-1}}(-m)\right|_{\mathbb{R P}^{2 m-1}} & \approx 2 m \mathcal{O}_{\mathbb{R P}^{2 m-1}}(1) \oplus 2 \mathcal{O}_{\mathbb{R P}^{2 m-1}}(1) \\
& \approx 2(m+1) \mathcal{O}_{\mathbb{R} \mathbb{P}^{2 m-1}}(1)
\end{aligned}
$$

The orientation on (2.32) induced by this relative spin structure agrees with the orientation induced by the relative spin structure associated with the oriented rank 2 real vector bundle $\mathcal{O}_{\mathbb{P} 2 m-1}(1)$ and 
the canonical spin structure on

$$
\left.2 m \mathcal{O}_{\mathbb{R}^{2 m-1}}(1) \oplus \mathcal{O}_{\mathbb{P}^{2 m-1}}(1)\right|_{\mathbb{R}^{2 m-1}} \approx 2(m+1) \mathcal{O}_{\mathbb{R}^{2 m-1}}(1)
$$

if and only if $m+1 \in 4 \mathbb{Z}$. Thus, the first and the third orientations on (2.32) are the same. The second relative spin structure is the relative spin structure of [3, Remark 6.5]. The claim now follows from [3, Remark 1.11].

The equality of the first and third orientations on (2.32) above can be seen in another way as well. The former is the orientation obtained as in [9] by adding $2 \mathcal{O}_{\mathbb{R P}^{2 m-1}}(-m)$ to $T \mathbb{P}^{2 m-1}$ and using the canonical spin structure on

$$
2 m \mathcal{O}_{\mathbb{R} \mathbb{P}^{2 m-1}}(1) \oplus 2 \mathcal{O}_{\mathbb{R}^{2 m-1}}(-m) \approx 2(m+1) \mathcal{O}_{\mathbb{R P}^{2 m-1}}(1) .
$$

By [12, Remark 3.10], the resulting orientation on (2.32) depends only on the latter (in contrast to the relative spin orientations). Thus, we can replace $\mathcal{O}_{\mathbb{R P}^{2 m-1}}(-m)$ by $\mathcal{O}_{\mathbb{R P}^{2 m-1}}(1)$. By [12, Corollary 3.8(1)], the resulting orientation on (2.32) agrees with the third orientation on (2.32).

Remark 2.7. The computation of the number (1.1) in [3] in both cases is confirmed through a second argument; see Corollary 5.4 and the paragraph above Remark 6.9 in [3]. It is also indirectly confirmed by the two proofs of [12, Proposition 3.5].

\section{Proof of Theorem 1.5}

We assume that $(X, \omega, \phi)$ is a compact real-orientable 6-manifold,

$$
B \in H_{2}(X ; \mathbb{Z})-\{0\}, \quad l, k \in \mathbb{Z}^{\geqslant 0}, \quad \text { and } \quad \mu_{1}, \ldots, \mu_{l} \in H^{*}(X ; \mathbb{Q}),
$$

are such that

$$
\sum_{i=1}^{l}\left(\operatorname{deg} \mu_{i}-2\right)+2 k=\left\langle c_{1}(X), B\right\rangle
$$

and either $k=0$ or

$$
\left\langle c_{1}(X), B^{\prime}\right\rangle \in 4 \mathbb{Z} \quad \forall B^{\prime} \in H_{2}(X ; \mathbb{Z}) \text { with } \phi_{*} B^{\prime}=-B^{\prime} \quad \text { and } \quad \mu_{1}, \ldots, \mu_{l}=\mathrm{PD}_{X}(\mathrm{pt}) .
$$

We fix $J \in \mathcal{J}_{\omega}^{\phi}$ as in the statement of Theorem 1.5.

\subsection{Real GW- and enumerative invariants}

For $g \in \mathbb{Z}^{\geqslant 0}$, denote by $\overline{\mathfrak{M}}_{g, l ; k}(B)$ the moduli space of real genus $g$ degree $B X$-valued $J$-holomorphic maps with $l$ conjugate pairs of marked points and $k$ real points and by

$$
\mathfrak{M}_{g, l ; k}(B) \subset \overline{\mathfrak{M}}_{g, l ; k}(B)
$$

the subspace of maps from smooth domains. The construction of Prym structures of [23] over $\mathbb{R}$ provides a real analogue of the perturbations $\nu$ of [31] over the Deligne-Mumford moduli $\overline{\mathcal{M}}_{g, l ; k}$ of real curves. For a $\phi$-invariant perturbation $\nu$, denote by $\overline{\mathfrak{M}}_{g, l ; k}(B ; \nu)$ the moduli space of real 
genus $g$ degree $B X$-valued $(J, \nu)$-holomorphic maps with $l$ conjugate pairs of marked points and $k$ real points and by

$$
\mathfrak{M}_{g, l ; k}(B ; \nu) \subset \overline{\mathfrak{M}}_{g, l ; k}(B ; \nu)
$$

the subspace of maps from smooth domains. Let

$$
\begin{aligned}
\mathrm{ev}: \mathfrak{M}_{g, l ; k}(B ; \nu) & \longrightarrow X^{l} \times\left(X^{\phi}\right)^{k} \\
{\left[u,\left(z_{1}^{+}, z_{1}^{-}\right), \ldots,\left(z_{l}^{+}, z_{l}^{-}\right), x_{1}, \ldots, x_{k}, \mathfrak{j}\right] } & \longrightarrow\left(u\left(z_{1}^{+}\right), \ldots, u\left(z_{l}^{+}\right), u\left(x_{1}\right), \ldots, u\left(x_{k}\right)\right),
\end{aligned}
$$

be the total evaluation map.

Choose a tuple $\mathbf{p} \equiv\left(p_{1}, \ldots, p_{k}\right) \in\left(X^{\phi}\right)^{k}$ of real points and pseudocycle representatives

$$
h_{1}: Y_{1} \longrightarrow X, \quad \ldots, \quad h_{l}: Y_{l} \longrightarrow X
$$

for the Poincare duals of $\mu_{1}, \ldots, \mu_{l}$; see the paragraph above [35, Theorem 1.1]. Define

$$
\begin{gathered}
\mathbf{h}=\left(h_{1}, \ldots, h_{l}\right): \mathbf{Y} \equiv \prod_{i=1}^{l} Y_{i} \longrightarrow X^{l}, \\
\Delta_{\mathbf{p}}^{l}=\left\{\left(q_{1}, \ldots, q_{l}, p_{1}, \ldots, p_{k}, q_{1}, \ldots, q_{l}\right): q_{1}, \ldots, q_{l} \in X\right\} \subset X^{l} \times\left(X^{\phi}\right)^{k} \times X^{l} .
\end{gathered}
$$

With $\nu$ as above, let

$$
\begin{gathered}
\overline{\mathfrak{M}}_{g, \mathbf{h} ; \mathbf{p}}(B ; \nu)=\left\{([\mathbf{u}], \mathbf{y}) \in \overline{\mathfrak{M}}_{g, l ; k}(B ; \nu) \times \mathbf{Y}:(\operatorname{ev}(\mathbf{u}), \mathbf{h}(\mathbf{y})) \in \Delta_{\mathbf{p}}^{l}\right\}, \\
\mathfrak{M}_{g, \mathbf{h} ; \mathbf{p}}(B ; \nu)=\overline{\mathfrak{M}}_{g, \mathbf{h} ; \mathbf{p}}(B ; \nu) \cap\left(\mathfrak{M}_{g, l ; k}(B ; \nu) \times \mathbf{Y}\right), \\
\overline{\mathfrak{M}}_{g, \mathbf{h} ; \mathbf{p}}(B)=\overline{\mathfrak{M}}_{g, \mathbf{h} ; \mathbf{p}}(B ; 0), \quad \mathfrak{M}_{g, \mathbf{h} ; \mathbf{p}}(B)=\mathfrak{M}_{g, \mathbf{h} ; \mathbf{p}}(B ; 0) .
\end{gathered}
$$

For generic choices of the tuple $\mathbf{p}$, the pseudocycle representatives $\mathbf{h}$, and a perturbation $\nu$, the map

$$
\mathrm{ev} \times \mathbf{h}: \overline{\mathfrak{M}}_{g, l ; k}(B ; \nu) \times \mathbf{Y} \longrightarrow X^{l} \times\left(X^{\phi}\right)^{k} \times X^{l}
$$

is transverse to $\Delta_{\mathbf{p}}^{l}$ on every stratum of the domain. In such a case,

$$
\mathfrak{M}_{g, \mathbf{h} ; \mathbf{p}}(B ; \nu)=\overline{\mathfrak{M}}_{g, \mathbf{h} ; \mathbf{p}}(B ; \nu)
$$

is a zero-dimensional orbifold oriented by a real orientation on $(X, \omega, \phi)$. For $g=1$, the corresponding real GW-invariant is the weighted cardinality of this orbifold, i.e.

$$
\mathrm{GW}_{1, B}^{\phi}\left(\mu_{1}, \ldots, \mu_{l} ; \mathrm{pt}^{k}\right) \equiv{ }^{ \pm}\left|\mathfrak{M}_{1, \mathbf{h} ; \mathbf{p}}(B ; \nu)\right| ;
$$

see [11, Theorem 1.4] and the proof of [11, Theorem 1.5]. This number is independent of the generic choices of $\nu, \mathbf{p}$, and $\mathbf{h}$, as well as of $J$.

By the genus 1 regularity assumption on $J$ and the proofs of [37, Propositions 1.7,1.8], the map

$$
\mathrm{ev} \times \mathbf{h}: \overline{\mathfrak{M}}_{g, l ; k}(B) \times \mathbf{Y} \longrightarrow X^{l} \times\left(X^{\phi}\right)^{k} \times X^{l}
$$


is also transverse to $\Delta_{\mathbf{p}}^{l}$ on every stratum of the domain for $g=0,1$ and generic choices of the tuple $\mathbf{p}$ and the pseudocycle representatives $\mathbf{h}$ and

$$
\mathfrak{M}_{g, \mathbf{h} ; \mathbf{p}}(B) \subset \overline{\mathfrak{M}}_{g, \mathbf{h} ; \mathbf{p}}(B)
$$

is a finite zero-dimensional manifold consisting of non-intersecting embeddings. A real orientation on $(X, \omega, \phi)$ and the proofs of [11, Theorems 1.4,1.5] endow this manifold with an orientation. For $g=1$, the corresponding real enumerative invariant is the signed cardinality of this manifold, i.e.

$$
\mathrm{E}_{1, B}^{\phi}\left(\mu_{1}, \ldots, \mu_{l} ; \mathrm{pt}^{k}\right) \equiv{ }^{ \pm}\left|\mathfrak{M}_{1, \mathbf{h} ; \mathbf{p}}(B)\right|
$$

We will show that

$$
\mathrm{GW}_{1, B}^{\phi}\left(\mu_{1}, \ldots, \mu_{l} ; \mathrm{pt}^{k}\right)=\mathrm{E}_{1, B}^{\phi}\left(\mu_{1}, \ldots, \mu_{l} ; \mathrm{pt}^{k}\right) ;
$$

the two sets in (3.2) may still be different. By (3.4), the number in (3.3) is independent of the generic choices of $\mathbf{p}$ and $\mathbf{h}$.

\subsection{Proof of (3.4)}

The proof of (3.4) is similar to the proof of [38, Theorem 1.1]. We denote by $\mathfrak{X}_{1, l ; k}(B)$ the space of all stable real genus 1 degree $d$ maps in the $L_{1}^{p}$-topology of [20, Section 3$]$ and by

$$
\mathfrak{X}_{1, l ; k}^{\{0\}}(B) \subset \mathfrak{X}_{1, l ; k}(B)
$$

the subspace of tuples $[\mathbf{u}]$ so that the degree of the restriction of the associated map $u$ to the principal component of the domain is nonzero. Let

$$
\begin{gathered}
\mathfrak{X}_{1, \mathbf{h} ; \mathbf{p}}(B)=\left\{([\mathbf{u}], \mathbf{y}) \in \mathfrak{X}_{1, l ; k}(B) \times \mathbf{Y}:(\operatorname{ev}(\mathbf{u}), \mathbf{h}(\mathbf{y})) \in \Delta_{\mathbf{p}}^{l}\right\}, \\
\mathfrak{X}_{1, \mathbf{h} ; \mathbf{p}}^{\{0\}}(B)=\mathfrak{X}_{1, \mathbf{h} ; \mathbf{p}}(B) \cap\left(\mathfrak{X}_{1, l ; k}^{\{0\}}(B) \times \mathbf{Y}\right) .
\end{gathered}
$$

By the genus 1 regularity assumption on $J$,

$$
\overline{\mathfrak{M}}_{1, \mathbf{h} ; \mathbf{p}}(B) \cap \mathfrak{X}_{1, \mathbf{h} ; \mathbf{p}}^{\{0\}}(B)=\mathfrak{M}_{1, \mathbf{h} ; \mathbf{p}}(B) .
$$

For $\nu$ sufficiently small, $\overline{\mathfrak{M}}_{1, \mathbf{h} ; \mathbf{p}}(B ; \nu)$ is contained in an arbitrarily small neighborhood of

$$
\overline{\mathfrak{M}}_{1, \mathbf{h} ; \mathbf{p}}(B) \subset \mathfrak{X}_{1, l ; k}(B) \times \mathbf{Y} .
$$

By the regularity of the subspace (3.2), there is a unique element of $\mathfrak{M}_{1, \mathbf{h} ; \mathbf{p}}(B ; \nu)$ near each element of $\mathfrak{M}_{1, \mathbf{h} ; \mathbf{p}}(B)$. Thus, the difference between the numbers in (3.4) is the number of elements of $\mathfrak{M}_{1, \mathbf{h} ; \mathbf{p}}(B ; \nu)$ that lie close to

$$
\overline{\mathfrak{M}}_{1, \mathbf{h} ; \mathbf{p}}(B)-\mathfrak{M}_{1, \mathbf{h} ; \mathbf{p}}(B) \subset \mathfrak{X}_{1, \mathbf{h} ; \mathbf{p}}(B)-\mathfrak{X}_{1, \mathbf{h} ; \mathbf{p}}^{\{0\}}(B) ;
$$

the inclusion above holds by (3.5).

By the regularity assumption on $J$, every map $u$ in the subset in (3.6) is not constant on a single bubble component $\Sigma_{u}^{*} \approx \mathbb{P}^{1}$ of its domain $\Sigma_{u}$. The image of $u$ is an embedded rational curve 
intersecting the pseudocycles $h_{1}, \ldots, h_{l}$ in distinct non-real points. Since $u$ is a real map and $\Sigma_{u}^{*}$ shares only one node $P_{u}$ with the remainder of $\Sigma_{u},\left.u\right|_{\Sigma_{u}^{*}}$ is a real map and $P_{u}$ is a non-isolated real node. The complement of $\Sigma_{u}^{*}$ in $\Sigma_{u}$ contains at most one real marked point (in addition to the node shared with $\Sigma_{u}^{*}$ ) and no conjugate pairs of marked points. If $\Sigma_{u}-\Sigma_{u}^{*}$ contains one marked point, then the restriction of $u$ to $\Sigma_{u}^{*}$ determines an element of

$$
\mathfrak{M}_{0, \mathbf{h} ; \mathbf{p}}(B)=\overline{\mathfrak{M}}_{0, \mathbf{h} ; \mathbf{p}}(B)
$$

with one of the real marked points distinguished and the remaining components of $\Sigma_{u}$ determine an element of $\overline{\mathcal{M}}_{1,0 ; 2}$. If $\Sigma_{u}-\Sigma_{u}^{*}$ contains no marked point, then the restriction of $u$ to $\Sigma_{u}^{*}$ determines an element of the preimage

$$
\mathfrak{M}_{0, \mathbf{h} ; \mathbf{p}}^{\prime}(B) \subset \mathfrak{M}_{0, l ; k+1}(B) \times \mathbf{Y}
$$

of $\mathfrak{M}_{0, \mathbf{h} ; \mathbf{p}}(B)$ under the forgetful morphism

$$
\mathfrak{f} \times \operatorname{id}_{\mathbf{Y}}: \mathfrak{M}_{0, l ; k+1}(B) \times \mathbf{Y} \longrightarrow \mathfrak{M}_{0, l ; k}(B) \times \mathbf{Y}
$$

dropping the additional marked point. The remaining components of $\Sigma_{u}$ determine an element of $\overline{\mathcal{M}}_{1,0 ; 1}$ in this case.

Since the images $\mathcal{C}_{u}$ of the elements of the $g=0$ spaces in (3.2) are disjoint real embedded curves in $(X, \phi)$, the topological components of $\overline{\mathfrak{M}}_{0, \mathbf{h} ; \mathbf{p}}^{\prime}(B)$ correspond to the elements $[\mathbf{u}]$ of the $g=0$ spaces in (3.2) . The evaluation

$$
\mathrm{ev}_{0}: \overline{\mathfrak{M}}_{0, \mathbf{h} ; \mathbf{p}}^{\prime}(B) \longrightarrow X^{\phi}
$$

at the distinguished real marked point associated with the node restricts to a diffeomorphism from each topological component of the domain to the real locus of the curve $\mathcal{C}_{u}$ corresponding to the given component.

The left-hand side of $(3.6)$ is stratified by the subspaces $\mathcal{U}_{\mathcal{T} ; \mathbf{h} ; \mathbf{p}}(B)$ of maps of a fixed combinatorial type $\mathcal{T}$. By the above, every non-empty stratum $\mathcal{U}_{\mathcal{T} ; \mathbf{h} ; \mathbf{p}}(B)$ is of the form

$$
\mathcal{U}_{\mathcal{T} ; \mathbf{h} ; \mathbf{p}}(B) \approx \mathcal{U}_{\mathcal{T}} \times \mathfrak{M}_{0, \mathbf{h} ; \mathbf{p}}^{\prime}(B),
$$

where $\mathcal{U}_{\mathcal{T}}$ is a stratum of $\overline{\mathcal{M}}_{1,0 ; 1}$, or of the form

$$
\mathcal{U}_{\mathcal{T} ; \mathbf{h} ; \mathbf{p}}(B) \approx \mathcal{U}_{\mathcal{T}} \times \mathfrak{M}_{0, \mathbf{h} ; \mathbf{p}}(B),
$$

where $\mathcal{U}_{\mathcal{T}}$ is a stratum of $\overline{\mathcal{M}}_{1,0 ; 2}$. In particular, the main boundary strata are of the form

$$
\mathcal{M}_{1,0 ; 1} \times \mathfrak{M}_{0, \mathbf{h} ; \mathbf{p}}^{\prime}(B) \quad \text { and } \quad \mathcal{M}_{1,0 ; 2} \times \mathfrak{M}_{0, \mathbf{h} ; \mathbf{p}}(B) .
$$

Proposition 3.1 below describes the signed number of elements of $\mathfrak{M}_{1, \mathbf{h} ; \mathbf{p}}(B ; \nu)$ near each stra$\operatorname{tum} \mathcal{U}_{\mathcal{T} ; \mathbf{h} ; \mathbf{p}}(B)$.

We denote by

$$
\mathbb{E}^{\mathbb{R}}, L_{1}^{\mathbb{R}} \longrightarrow \overline{\mathcal{M}}_{1,0 ; 1} \quad \text { and } \quad \mathbb{E}^{\mathbb{R}}, L_{1}^{\mathbb{R}} \longrightarrow \overline{\mathcal{M}}_{1,0 ; 2}
$$

the real parts of the Hodge line bundle of holomorphic differentials and of the universal tangent line bundle at the marked point and their pullbacks by the forgetful morphism. The real part of 
the tautological line bundle over $\overline{\mathcal{M}}_{1,0 ; 2}$ for the first marked point and $L_{1}^{\mathbb{R}}$ are canonically identified over $\mathcal{M}_{1,0 ; 2}$. Let

$$
L_{0}^{\mathbb{R}} \longrightarrow \overline{\mathfrak{M}}_{0,0 ;\{\mathbf{0}\}}(B) \quad \text { and } \quad L_{0}^{\mathbb{R}} \longrightarrow \mathfrak{M}_{0, \mathbf{h} ; \mathbf{p}}(B), \overline{\mathfrak{M}}_{0, \mathbf{h} ; \mathbf{p}}^{\prime}(B)
$$

denote the real part of the universal tangent line bundle at the marked point and its pullbacks by the projection map to the moduli space component and the forgetful morphisms (keeping only the marked point associated with the node).

The homomorphism

$$
s_{1}: L_{1}^{\mathbb{R}} \longrightarrow\left(\mathbb{E}^{\mathbb{R}}\right)^{*}, \quad\left\{s_{1}(v)\right\}(\psi)=\psi(v),
$$

of real line bundles over $\overline{\mathcal{M}}_{1,0 ; 1}$ and $\overline{\mathcal{M}}_{1,0 ; 2}$ is an isomorphism. We define a bundle homomorphism over $\overline{\mathfrak{M}}_{0,0 ;\{\mathbf{0}\}}(B), \mathfrak{M}_{0, \mathbf{h} ; \mathbf{p}}(B)$, and $\overline{\mathfrak{M}}_{0, \mathbf{h} ; \mathbf{p}}^{\prime}(B)$ by

$$
\mathcal{D}_{0}^{\mathbb{R}}: L_{0}^{\mathbb{R}} \longrightarrow \mathrm{ev}_{0}^{*} T X^{\phi}, \quad \mathcal{D}_{0}^{\mathbb{R}}([\mathbf{u}, v])=\mathrm{d}_{x_{0}(\mathbf{u})} u(v),
$$

where $x_{0}(\mathbf{u})$ is the distinguished marked point and $u$ is the map component of the tuple $\mathbf{u}$. The restriction of (3.10) over the component of $\overline{\mathfrak{M}}_{0, \mathbf{h} ; \mathbf{p}}^{\prime}(B)$ corresponding to an embedded real curve $\mathcal{C}$ in $(X, \phi)$ identifies $L_{0}^{\mathbb{R}}$ with $\mathrm{ev}_{0}^{*} T \mathcal{C}^{\phi}$. For each combinatorial type $\mathcal{T}$ as above, let $\mathcal{D}_{\mathcal{T}}$ be the bundle homomorphism over $\mathcal{U}_{\mathcal{T} ; \mathbf{h} ; \mathbf{p}}(B)$ given by

$$
\mathcal{D}_{\mathcal{T}}=\pi_{1}^{*} s_{1} \otimes_{\mathbb{R}} \pi_{2}^{*} \mathcal{D}_{0}^{\mathbb{R}}: \pi_{1}^{*} L_{1}^{\mathbb{R}} \otimes_{\mathbb{R}} \pi_{2}^{*} L_{0}^{\mathbb{R}} \longrightarrow \pi_{1}^{*}\left(\mathbb{E}^{\mathbb{R}}\right)^{*} \otimes_{\mathbb{R}} \pi_{2}^{*} \mathrm{ev}_{0}^{*} T X^{\phi}
$$

with respect to the decomposition (3.7) or (3.8).

For a generic choice of a bundle section $\bar{\nu}_{\mathcal{T}}$ of

$$
\pi_{1}^{*}\left(\mathbb{E}^{\mathbb{R}}\right)^{*} \otimes_{\mathbb{R}} \pi_{2}^{*} \operatorname{ev}_{0}^{*} T X^{\phi} \longrightarrow \overline{\mathcal{U}}_{\mathcal{T} ; \mathbf{h} ; \mathbf{p}}(B),
$$

the affine bundle map

$$
\begin{gathered}
\alpha_{\mathcal{T}, \bar{\nu}_{\mathcal{T}}}: \pi_{1}^{*} L_{1}^{\mathbb{R}} \otimes_{\mathbb{R}} \pi_{2}^{*} L_{0}^{\mathbb{R}} \longrightarrow \pi_{1}^{*}\left(\mathbb{E}^{\mathbb{R}}\right)^{*} \otimes_{\mathbb{R}} \pi_{2}^{*} \operatorname{ev}_{0}^{*} T X^{\phi} \\
\alpha_{\mathcal{T}, \bar{\nu}_{\mathcal{T}}}(v)=\mathcal{D}_{\mathcal{T}}(v)+\left.\bar{\nu}_{\mathcal{T}}(\mathbf{u}) \quad \forall v \in \pi_{1}^{*} L_{1}^{\mathbb{R}} \otimes_{\mathbb{R}} \pi_{2}^{*} L_{0}^{\mathbb{R}}\right|_{\mathbf{u}}, \quad \mathbf{u} \in \mathcal{U}_{\mathcal{T} ; \mathbf{h} ; \mathbf{p}}(B),
\end{gathered}
$$

over $\overline{\mathcal{U}}_{\mathcal{T} ; \mathbf{h} ; \mathbf{p}}(B)$ is transverse to the zero set. This section thus has no zeros unless $\mathcal{T}$ corresponds to one of the main strata (3.9). Since $\mathcal{D}_{\mathcal{T}}$ is injective, $\alpha_{\mathcal{T}, \bar{\nu} \mathcal{T}}$ has a finite number of transverse zeros over each main stratum.

The affine bundle map $\alpha_{\mathcal{T}, \bar{\nu}_{\mathcal{T}}}$ can be viewed as a section $\alpha_{\mathcal{T}, \bar{\nu}_{\mathcal{T}}}^{\prime}$ of the bundle

$$
\pi_{\mathcal{T}}^{*}\left(\pi_{1}^{*}\left(\mathbb{E}^{\mathbb{R}}\right)^{*} \otimes_{\mathbb{R}} \pi_{2}^{*} \mathrm{ev}_{0}^{*} T X^{\phi}\right) \longrightarrow \pi_{1}^{*} L_{1}^{\mathbb{R}} \otimes_{\mathbb{R}} \pi_{2}^{*} L_{0}^{\mathbb{R}},
$$

where

$$
\pi_{\mathcal{T}}: \pi_{1}^{*} L_{1}^{\mathbb{R}} \otimes_{\mathbb{R}} \pi_{2}^{*} L_{0}^{\mathbb{R}} \longrightarrow \overline{\mathcal{U}}_{\mathcal{T} ; \mathbf{h} ; \mathbf{p}}(B)
$$

is the bundle projection map. By our assumptions, the choice of a real orientation on $(X, \omega, \phi)$ used to define the number (3.1) orients the uncompactified moduli space $\mathfrak{M}_{1, \mathbf{h} ; \mathbf{p}}(B)$. Since the 
domain and target of $\alpha_{\mathcal{T}, \bar{\nu}_{\mathcal{T}}}$ form a deformation-obstruction complex for the oriented moduli space $\overline{\mathfrak{M}}_{1, \mathbf{h} ; \mathbf{p}}(B)$ over the main strata $\mathcal{U}_{\mathcal{T} ; \mathbf{h} ; \mathbf{p}}(B)$ and all zeros of $\alpha_{\mathcal{T}, \overline{\mathcal{T}}}^{\prime}$ are contained in

$$
\left.\pi_{\mathcal{T}}^{*}\left(\pi_{1}^{*}\left(\mathbb{E}^{\mathbb{R}}\right)^{*} \otimes_{\mathbb{R}} \pi_{2}^{*} \operatorname{ev}_{0}^{*} T X^{\phi}\right)\right|_{\left.\pi_{1}^{*} L_{1}^{\mathbb{R}} \otimes_{\mathbb{R}} \pi_{2}^{*} L_{0}^{\mathbb{R}}\right|_{\mathcal{U} ; \mathbf{h} ; \mathbf{p}}(B)}-\pi_{1}^{*} L_{1}^{\mathbb{R}} \otimes_{\mathbb{R}} \pi_{2}^{*} L_{0}^{\mathbb{R}},
$$

the orientation on $\mathfrak{M}_{1, \mathbf{h} ; \mathbf{p}}(B)$ determines a sign for each zero of $\alpha_{\mathcal{T}, \bar{\nu}_{\mathcal{T}}}^{\prime}$ and $\alpha_{\mathcal{T}, \bar{\nu}_{\mathcal{T}}}$ (these are the same). We denote the resulting weighted cardinality of $\alpha_{\mathcal{T}, \bar{\nu} \mathcal{T}}^{-1}(0)$ by $N\left(\mathcal{D}_{\mathcal{T}}, \bar{\nu}_{\mathcal{T}}\right)$.

As described above [38, (3.14)], a perturbation $\nu$ as above determines a section $\bar{\nu}_{\mathcal{T}}$ of the complex analogue of the bundle (3.11). If $\nu$ is $\phi$-invariant, then $\bar{\nu}_{\mathcal{T}}$ is a section of (3.11). If $\nu$ is generic, then $\alpha_{\mathcal{T}, \bar{\nu}_{\mathcal{T}}}$ is transverse to the zero set for every $\mathcal{T}$.

Proposition 3.1. There exists an non-empty subspace of $\phi$-invariant perturbations $\nu$ satisfying the following property. For each boundary stratum $\mathcal{U}_{\mathcal{T} ; \mathbf{h} ; \mathbf{p}}(B)$ of $\overline{\mathfrak{M}}_{1, \mathbf{h} ; \mathbf{p}}(B)$, there exist $\mathcal{C}_{\mathcal{T}}\left(\bar{\partial}_{J}\right) \in \mathbb{Q}$ and a compact subset $K_{\nu}$ of $\mathcal{U}_{\mathcal{T} ; \mathbf{h} ; \mathbf{p}}(B)$ with the following property. For every compact subset $K$ of $\mathcal{U}_{\mathcal{T} ; \mathbf{h} ; \mathbf{p}}(B)$ and open subset $U$ of $\mathfrak{X}_{1, \mathbf{h} ; \mathbf{p}}(B)$, there exist an open neighborhood $U_{\nu}(K)$ of $K$ in $\mathfrak{X}_{1, \mathbf{h} ; \mathbf{p}}(B)$ and $\epsilon_{\nu}(U) \in \mathbb{R}^{+}$, respectively, such that

$$
{ }^{ \pm}\left|\mathfrak{M}_{1, \mathbf{h} ; \mathbf{p}}(B ; t \nu) \cap U\right|=\mathcal{C}_{\mathcal{T}}\left(\bar{\partial}_{J}\right) \quad \text { if } t \in\left(0, \epsilon_{\nu}(U)\right), \quad K_{\nu} \subset K \subset U \subset U_{\nu}(K) .
$$

Furthermore, $\mathcal{C}_{\mathcal{T}}\left(\bar{\partial}_{J}\right)=N\left(\mathcal{D}_{\mathcal{T}}, \bar{\nu}_{\mathcal{T}}\right)$ if $\mathcal{U}_{\mathcal{T} ; \mathbf{h} ; \mathbf{p}}(B)$ is a main stratum and $\mathcal{C}_{\mathcal{T}}\left(\bar{\partial}_{J}\right)=0$ otherwise.

This is the real case of [38, Proposition 3.1]. It is obtained by restricting the proof in [38] to the space of real parameters.

It remains to compute the number $N\left(\mathcal{D}_{\mathcal{T}}, \bar{\nu}_{\mathcal{T}}\right)$ for each of the main strata (3.9). Since the section $\mathcal{D}_{\mathcal{T}}$ is injective, the zeros of $\alpha_{\mathcal{T}, \bar{\nu}_{\mathcal{T}}}$ correspond to the zeros of the transverse bundle section $\bar{\nu}_{\mathcal{T}}^{\prime}$ of

$$
\pi_{1}^{*}\left(\mathbb{E}^{\mathbb{R}}\right)^{*} \otimes_{\mathbb{R}} \pi_{2}^{*} \operatorname{ev}_{0}^{*} T X^{\phi} / \operatorname{Im} \mathcal{D}_{\mathcal{T}} \approx \pi_{1}^{*} L_{1}^{\mathbb{R}} \otimes_{\mathbb{R}} \pi_{2}^{*}\left(\operatorname{ev}_{0}^{*} T X^{\phi} / \operatorname{Im} \mathcal{D}_{0}\right)
$$

obtained by composting $\bar{\nu}_{\mathcal{T}}^{\prime}$ with the projection map; see [33, Section 3.3].

Each topological component of the closure $\overline{\mathcal{U}}_{\mathcal{T} ; \mathbf{h} ; \mathbf{p}}(B)$ of the first space in (3.9) is $S^{1} \times S^{1}$, with the circles coming from each factor. By the proof of [11, Theorem 1.5], the orientation on $\mathfrak{M}_{1, \mathbf{h} ; \mathbf{p}}(B)$ extends to an orientation over $\overline{\mathcal{U}}_{\mathcal{T} ; \mathbf{h} ; \mathbf{p}}(B)$. This orientation and an orientation on $\overline{\mathcal{U}}_{\mathcal{T} ; \mathbf{h} ; \mathbf{p}}(B)$ induce an orientation on the target vector bundle in (3.12). The number $N\left(\mathcal{D}_{\mathcal{T}}, \bar{\nu}_{\mathcal{T}}\right)$ is the signed number of zeros of the section $\bar{\nu}_{\mathcal{T}}^{\prime}$ with respect to the chosen orientation on $\overline{\mathcal{U}}_{\mathcal{T} ; \mathbf{h} ; \mathbf{p}}(B)$ and the induced orientation on this vector bundle, i.e.

$$
N\left(\mathcal{D}_{\mathcal{T}}, \bar{\nu}_{\mathcal{T}}\right)=\left\langle e\left(\pi_{1}^{*} L_{1}^{\mathbb{R}} \otimes_{\mathbb{R}} \pi_{2}^{*}\left(\operatorname{ev}_{0}^{*} T X^{\phi} / \operatorname{Im} \mathcal{D}_{0}\right)\right),\left[\overline{\mathcal{U}}_{\mathcal{T} ; \mathbf{h} ; \mathbf{p}}(B)\right]\right\rangle .
$$

Since the restriction of $\operatorname{ev}_{0}^{*} T X^{\phi} / \operatorname{Im} \mathcal{D}_{0}$ to the component of $\overline{\mathfrak{M}}_{0, \mathbf{h} ; \mathbf{p}}^{\prime}(B)$ corresponding to a curve $\mathcal{C}_{u} \subset X$ is the normal bundle of $\mathcal{C}_{u}^{\phi}$ in $X^{\phi}$ and is thus orientable, the number (3.13) is zero in this case.

Each topological component of the closure of the second space in (3.9) is $\overline{\mathcal{M}}_{1,0 ; 2}$ and

$$
\pi_{1}^{*} L_{1}^{\mathbb{R}} \otimes_{\mathbb{R}} \pi_{2}^{*}\left(\operatorname{ev}_{0}^{*} T X^{\phi} / \operatorname{Im} \mathcal{D}_{0}\right) \approx L_{1}^{\mathbb{R}} \oplus L_{1}^{\mathbb{R}} \longrightarrow \overline{\mathcal{M}}_{1,0 ; 2}
$$


in this case. Since $L_{1}^{\mathbb{R}}$ is the pullback of a bundle over $\overline{\mathcal{M}}_{1,0 ; 1}$ in our setup, there is an open subset of sections $\bar{\nu}_{\mathcal{T}}^{\prime}$ of (3.14) that have no zeros. Thus, $\nu$ can be chosen so that the section $\alpha_{\mathcal{T}, \bar{\nu}_{\mathcal{T}}}$ has no zeros and so $N\left(\mathcal{D}_{\mathcal{T}}, \bar{\nu}_{\mathcal{T}}\right)=0$ in this case as well.

In summary, the difference between the two numbers in $(3.4)$ is the sum of the numbers $N\left(\mathcal{D}_{\mathcal{T}}, \bar{\nu}_{\mathcal{T}}\right)$ corresponding to the main boundary strata of $\overline{\mathfrak{M}}_{1, \mathbf{h} ; \mathbf{p}}(B)$. By the last two paragraphs, each of these numbers $N\left(\mathcal{D}_{\mathcal{T}}, \bar{\nu}_{\mathcal{T}}\right)$ is zero. This establishes (3.4) and Theorem 1.5.

\section{Equivariant localization}

Throughout this section, $n \in \mathbb{Z}^{+}, m$ is the integer part of $n / 2$, and

$$
[n]=\{1, \ldots, n\} .
$$

We denote by $\phi$ either the involution $\tau_{n}^{\prime}$ on $\mathbb{P}^{n-1}$ given by (2.2) or the involution $\eta_{n}$ on $\mathbb{P}^{n-1}$ with $n=2 m$ given by (1.2). The restriction of the standard $\mathbb{T}^{n}$-action on $\mathbb{P}^{n-1}$ to a subtorus $\mathbb{T}^{m} \subset \mathbb{T}^{n}$ commutes with the involution $\phi$; see Section 4.1. This restriction thus induces a $\mathbb{T}^{m}$-action on the moduli space $\overline{\mathfrak{M}}_{g, l}\left(\mathbb{P}^{n-1}, d\right)^{\phi}$ of genus $g$ degree $d$ real $J_{0}$-holomorphic maps into $\left(\mathbb{P}^{n-1}, \phi\right)$ with $l$ pairs of conjugate marked points. We describe the fixed loci of this action and their normal bundles, as real vector bundles, in Sections 5.1 and 5.2. By Proposition 1.4, $\left(\mathbb{P}^{n-1}, \phi\right)$ admits a real orientation if $(n, \phi)=\left(2 m, \tau_{n}^{\prime}\right)$ or $(n, \phi)=\left(4 m, \eta_{n}\right)$. By [11, Theorem 1.3], $\overline{\mathfrak{M}}_{g, l}\left(\mathbb{P}^{n-1}, d\right)^{\phi}$ is orientable in these cases and is oriented by a real orientation on $\left(\mathbb{P}^{n-1}, \phi\right)$. We determine the orientations on the normal bundles to the fixed loci with respect to the real orientations of Section 2.2 in Section 5.3. The equivariant localization data provided by Theorem 4.6 determines the real GW-invariants of $\left(\mathbb{P}^{2 m-1}, \omega_{2 n}, \tau_{2 m}\right)$ and $\left(\mathbb{P}^{4 m-1}, \omega_{4 m}, \eta_{4 m}\right)$ and exhibits the two types of vanishing phenomena described in [32, Sections 3.2,3.3]. We illustrate their use in Section 4.3,

We also describe equivariant localization data related to the real GW-invariants of the complete intersections $X_{n ; \mathbf{a}} \subset \mathbb{P}^{n-1}$ preserved by $\phi$. Such a complete intersection is the zero set of a transverse holomorphic section $s_{n ; \mathbf{a}}$ of the vector bundle (2.27) satisfying $s_{n ; \mathbf{a}} \circ \phi=\widetilde{\phi}_{n ; \mathbf{a}} \circ s_{n ; \mathbf{a}}$ for a conjugation $\widetilde{\phi}_{n ; \mathbf{a}}$ lifting $\phi$. The latter is necessarily a direct sum of the conjugations $\widetilde{\phi}_{n ; 1}^{(a)}$ and $\widetilde{\phi}_{n ; 1,1}^{(a)}$ described in Section 2.1. Let $\phi_{n ; \mathbf{a}}=\left.\phi\right|_{X_{n ; \mathbf{a}}}$. If $(n, \mathbf{a})$ and $\phi$ satisfy the assumptions of Proposition 1.4 (with $\tau_{n}$ replaced by $\tau_{n}^{\prime}$ in the first case), then $\left(X_{n ; \mathbf{a}}, \phi_{n ; \mathbf{a}}\right)$ admits a real orientation. By [11, Theorem 1.3], $\overline{\mathfrak{M}}_{g, l}\left(X_{n ; \mathbf{a}}, d\right)^{\phi_{n ; \mathbf{a}}}$ is then orientable if $n-k \in 2 \mathbb{Z}$ (so that the complex dimension of $X_{n ; \mathbf{a}}$ is odd) and is oriented by a real orientation on $\left(X_{n ; \mathbf{a}}, \phi_{n ; \mathbf{a}}\right)$.

Whenever defined, the real GW-invariants of $\left(X_{n ; \mathbf{a}}, \omega_{2 n ; \mathbf{a}}, \phi_{n ; \mathbf{a}}\right)$ are expected to be related to the "fibration"

$$
\pi_{n ; \mathbf{a}}^{\widetilde{\phi}_{n ; \mathbf{a}}}: \mathcal{V}_{n ; \mathbf{a}}^{\widetilde{\phi}_{n ; \mathbf{a}}} \equiv \overline{\mathfrak{M}}_{g, l}\left(\mathcal{L}_{n ; \mathbf{a}}, d\right)^{\widetilde{\phi}_{n ; \mathbf{a}}} \longrightarrow \overline{\mathfrak{M}}_{g, l}\left(\mathbb{P}^{n-1}, d\right)^{\phi} .
$$

The $\mathbb{T}^{m}$-action on the base of this "fibration" lifts canonically to the total space. The restriction of (4.1) to a certain open subspace

$$
\mathfrak{M}_{g, l}^{\star}\left(\mathbb{P}^{n-1}, d\right)^{\phi} \subset \overline{\mathfrak{M}}_{g, l}\left(\mathbb{P}^{n-1}, d\right)^{\phi}
$$

is a vector orbi-bundle of the expected rank, but the dimensions of the fibers of (4.1) are higher over the complement of (4.2). Under the assumptions of Proposition 1.4, the orientation systems 
of the restrictions of $\mathcal{V}_{n ; \mathbf{a}}^{\widetilde{\phi}_{n} \mathbf{a}}$ and $T \overline{\mathfrak{M}}_{g, l}\left(\mathbb{P}^{n-1}, d\right)^{\phi}$ to $\mathfrak{M}_{g, l}^{\star}\left(\mathbb{P}^{n-1}, d\right)^{\phi}$ are the same. The section $s_{n ; \mathbf{a}}$ and the real orientation on $\left(X_{n ; \mathbf{a}}, \phi_{n ; \mathbf{a}}\right)$ described in Section 2.2 determine a homotopy class of isomorphisms between these two systems and a class

$$
\mathbf{e}\left(\mathcal{V}_{n ; \mathbf{a}}^{\widetilde{\phi}_{n ; \mathbf{a}}}\right) \in H_{\mathbb{T}^{m}}^{*}\left(\mathfrak{M}_{g, l}^{\star}\left(\mathbb{P}^{n-1}, d\right)^{\phi} ; \mathfrak{O}\right)
$$

in the $\mathbb{T}^{m}$-equivariant cohomology with coefficients in the orientation system of $\overline{\mathfrak{M}}_{g, l}\left(\mathbb{P}^{n-1}, d\right)^{\phi}$.

For each $i=1, \ldots, l$, let

$$
\psi_{i} \in H^{2}\left(\overline{\mathfrak{M}}_{g, l}\left(\mathbb{P}^{n-1}, d\right)^{\phi} ; \mathbb{Q}\right)
$$

denote the first Chern class of the universal cotangent line bundle associated with the first marked point in the $i$-th conjugate pair. In Section 4.2, we describe the equivariant localization contributions to

$$
\int_{\left[\overline{\mathfrak{M}}_{g, l}\left(\mathbb{P}^{n-1}, d\right)^{\phi}\right]^{\mathrm{vrt}}} \prod_{i=1}^{l}\left(\psi_{i}^{b_{i}} \mathrm{ev}_{i}^{*} \mathbf{x}^{p_{i}}\right) \mathbf{e}\left(\mathcal{V}_{n ; \mathbf{a}}^{\widetilde{\phi}_{n}}\right) \in H_{\mathbb{T}^{m}}^{*}
$$

for any extension of (4.3) to a class

$$
\mathbf{e}\left(\mathcal{V}_{n ; \mathbf{a}}^{\widetilde{\phi}_{n ; \mathbf{a}}}\right) \in H_{\mathbb{T}^{m}}^{*}\left(\overline{\mathfrak{M}}_{g, l}\left(\mathbb{P}^{n-1}, d\right)^{\phi} ; \mathfrak{O}\right)
$$

from the $\mathbb{T}^{m}$-fixed loci contained in $\mathfrak{M}_{g, l}^{\star}\left(\mathbb{P}^{n-1}, d\right)^{\phi}$; all such loci consist of real maps with no contracted positive-genus components (unless $k=0$, i.e. $\left.X_{n ; \mathbf{a}}=\mathbb{P}^{n-1}\right)$. For $(n, \mathbf{a})=(5,(5))$ and $\phi=\tau_{n}^{\prime}$, our conclusion specializes to [32, (3.22)]. The equivariant contributions to (4.4) described by Theorem 4.6 vanish whenever the orientation on $\overline{\mathfrak{M}}_{g, l}\left(X_{n ; \mathbf{a}}, d\right)^{\phi_{n ; \mathbf{a}}}$ induced by the canonical real orientation depends on the implicit choices made in Section 2.2, see Section 2.3 .

If $k=0$ or $g=0$, the subspace in (4.2) is the entire moduli space. In the first case, $\mathbf{e}\left(\mathcal{V}_{n ; \mathbf{a}}\right)=1$. In the second case, the class (4.3) relates the $g=0$ real GW-invariants of $\left(X_{n ; \mathbf{a}}, \omega_{n ; \mathbf{a}}, \phi_{n ; \mathbf{a}}\right)$ to $\left(\mathbb{P}^{n-1}, \omega_{n}, \phi\right)$, as shown in the proof of [28, Theorem 3] for $(n, \mathbf{a})=(5,(5))$ and $\phi=\tau_{n}^{\prime}$. If $g \geqslant 1$ and $k \geqslant 1$, the subspace in (4.2) is not even dense in the entire moduli space. By [34, Theorem 1.1] and [21, Theorem 1.1], the natural extension of the complex analogue of the $g=1$ case of (4.3) is a class on the closure

$$
\overline{\mathfrak{M}}_{1, l}^{0}\left(\mathbb{P}^{n-1}, d\right) \subset \overline{\mathfrak{M}}_{1, l}\left(\mathbb{P}^{n-1}, d\right)
$$

of the subspace corresponding to (4.2) and relates the reduced genus 1 (complex) GW-invariants of $X_{n ; \mathbf{a}}$ defined in [38] to $\left(\mathbb{P}^{n-1}, \omega_{n}\right)$. By [36, Theorem 1A], the difference between the standard and reduced GW-invariants of $X_{n ; \mathbf{a}}$ is a combination of the genus $0 \mathrm{GW}$-invariants. The same should be the case in the real setting and in higher genera. The proof of [38, Theorem 1.1] in fact suggests that the real analogues of the reduced genus $1 \mathrm{GW}$-invariants are equal to the genus 1 real GW-invariants of $(X, \omega, \phi)$ defined in [11] if $\operatorname{dim}_{\mathbb{R}} X=6$. This is consistent with the prediction of [32, Section 3.4] that the genus 1 real GW-invariants of $\left(X_{n ; \mathbf{a}}, \omega_{n ; \mathbf{a}}, \tau_{n ; \mathbf{a}}^{\prime}\right)$ are obtained from the equivariant contributions of the $\mathbb{T}^{m}$-fixed loci in $\mathfrak{M}_{g, l}^{\star}\left(\mathbb{P}^{n-1}, d\right)^{\phi}$ described in Section 4.2.

\subsection{Equivariant setting}

Let $\mathbb{T}^{n}$ denote the $n$-torus $\left(S^{1}\right)^{n}$ or its complex analogue $\left(\mathbb{C}^{*}\right)^{n}$. The $\mathbb{T}^{n}$-quotient of its classifying space $E \mathbb{T}^{n}$ is $B \mathbb{T}^{n}=\left(\mathbb{P}^{\infty}\right)^{n}$. Thus, the group cohomology of $\mathbb{T}^{n}$ is

$$
H_{\mathbb{T}^{n}}^{*} \equiv H^{*}\left(B \mathbb{T}^{n} ; \mathbb{Q}\right)=\mathbb{Q}\left[\alpha_{1}, \ldots, \alpha_{n}\right]
$$


where $\alpha_{i} \equiv \pi_{i}^{*} c_{1}\left(\gamma_{\infty}^{*}\right), \gamma_{\infty} \longrightarrow \mathbb{P}^{\infty}$ is the tautological line bundle, and

$$
\pi_{i}:\left(\mathbb{P}^{\infty}\right)^{n} \longrightarrow \mathbb{P}^{\infty}
$$

is the projection to the $i$-th component. We will call $\alpha_{1}, \ldots, \alpha_{n}$ the weights of the standard representation of $\mathbb{T}^{n}$ on $\mathbb{C}^{n}$. Let

$$
\mathcal{H}_{\mathbb{T}^{n}}^{*} \approx \mathbb{Q}\left(\alpha_{1}, \ldots, \alpha_{n}\right)
$$

denote the field of fractions of $H_{\mathbb{T}^{n}}^{*}$.

We denote the $\mathbb{T}^{n}$-equivariant $\mathbb{Q}$-cohomology of a topological space $M$ with a $\mathbb{T}^{n}$-action, i.e. the cohomology of

$$
B_{\mathbb{T}^{n}} M \equiv E \mathbb{T}^{n} \times_{\mathbb{T}^{n}} M,
$$

by $H_{\mathbb{T}^{n}}^{*}(M)$. The projection $B_{\mathbb{T}^{n}} M \longrightarrow B \mathbb{T}^{n}$ induces an action of $H_{\mathbb{T}^{n}}^{*}$ on $H_{\mathbb{T}^{n}}^{*}(M)$. Define

$$
\mathcal{H}_{\mathbb{T}^{n}}^{*}(M)=\mathcal{H}_{\mathbb{T}^{n}}^{*} \otimes_{H_{\mathbb{T}^{n}}^{*}} H_{\mathbb{T}^{n}}^{*}(M) .
$$

If $\mathbb{T}^{n}$ acts trivially on $M$, then

$$
B_{\mathbb{T}^{n}} M=B \mathbb{T}^{n} \times M, \quad \mathcal{H}_{\mathbb{T}^{n}}^{*}(M)=\mathcal{H}_{\mathbb{T}^{n}}^{*} \otimes H^{*}(M ; \mathbb{Q}) ;
$$

the last identification is on the level of $\mathcal{H}_{\mathbb{T}^{n}}^{*}$-algebras. For an oriented vector bundle $V \longrightarrow M$ with a $\mathbb{T}^{n}$-action lifting the action on $M$, let

$$
\mathbf{e}(V) \equiv e\left(B_{\mathbb{T}^{n}} V\right) \in H_{\mathbb{T}^{n}}^{*}(M)
$$

denote the equivariant Euler class of $V$. For a complex bundle $V \longrightarrow M$ with a $\mathbb{T}^{n}$-action lifting the action on $M$, let

$$
\mathbf{c}(V) \equiv c\left(B_{\mathbb{T}^{n}} V\right) \in H_{\mathbb{T}^{n}}^{*}(M)
$$

denote the equivariant Chern class of $V$. A continuous $\mathbb{T}^{n}$-equivariant map $f: M^{\prime} \longrightarrow M$ between two topological spaces induces a homomorphism

$$
f^{*}: H_{\mathbb{T}}^{*}(M) \longrightarrow H_{\mathbb{T}}^{*}\left(M^{\prime}\right) .
$$

The equivariant Euler and Chern classes are natural with respect to such maps.

The standard action of $\mathbb{T}^{n}$ on $\mathbb{C}^{n}$,

$$
\left(u_{1}, \ldots, u_{n}\right) \cdot\left(z_{1}, \ldots, z_{n}\right)=\left(u_{1} z_{1}, \ldots, u_{n} z_{n}\right)
$$

descends to a $\mathbb{T}^{n}$-action on $\mathbb{P}^{n-1}$. The latter has $n$ fixed points,

$$
P_{1} \equiv[1,0, \ldots, 0], \quad \ldots, \quad P_{n} \equiv[0, \ldots, 0,1] .
$$

The curves preserved by this action are the lines through the fixed points,

$$
\mathbb{P}_{i j}^{1} \equiv\left\{\left[Z_{1}, \ldots, Z_{n}\right] \in \mathbb{P}^{n-1}: Z_{k}=0 \forall k \neq i, j\right\}, \quad i, j \in[n], i \neq j .
$$

The product of the standard $\mathbb{T}^{n}$-actions on $\mathbb{P}^{n-1}$ and $\mathbb{C}^{n}$ restricts to a $\mathbb{T}^{n}$-action on the tautological line bundle

$$
\gamma_{n-1} \subset \mathbb{P}^{n-1} \times \mathbb{C}^{n}
$$


as in (2.4) and induces $\mathbb{T}^{n}$-actions on the holomorphic line bundles

$$
\mathcal{O}_{\mathbb{P}^{n-1}}(a) \equiv\left(\gamma_{n-1}^{*}\right)^{\otimes a}, \quad \forall a \in \mathbb{Z}
$$

Let

$$
\mathbf{x} \equiv \mathbf{e}\left(\gamma_{n-1}^{*}\right) \in H_{\mathbb{T}^{n}}^{*}\left(\mathbb{P}^{n-1}\right)
$$

denote the equivariant hyperplane class. The equivariant restrictions of $\mathbf{x}$ and $\mathbf{c}\left(T \mathbb{P}^{n-1}\right)$ to the fixed points (4.5) are described by

$$
\left.\mathbf{x}\right|_{P_{i}}=\alpha_{i} \in H_{\mathbb{T}^{n}}^{*}=H_{\mathbb{T}^{n}}^{*}\left(P_{i}\right),\left.\quad \mathbf{c}\left(T \mathbb{P}^{n-1}\right)\right|_{P_{i}}=\prod_{k \neq i}\left(1+\alpha_{i}-\alpha_{k}\right) \quad \forall i \in[n] .
$$

The first identity above follows from the definition of $\alpha_{i}$. The second identity follows from the homomorphisms $f$ and $g$ in the short exact sequence (2.7) being $\mathbb{T}^{n}$-equivariant with respect to the $\mathbb{T}^{n}$-action on the middle term obtained by tensoring the standard action on $\gamma_{n-1}^{*}$ with the standard action on $\mathbb{C}^{n}$.

With notation as in (2.5),

$$
\phi\left(P_{i}\right)=P_{\phi(i)} .
$$

Denote by $\lambda_{1}, \ldots, \lambda_{m}$ the weights of the standard representation of $\mathbb{T}^{m}$ on $\mathbb{C}^{m}$. The embedding

$$
\iota: \mathbb{T}^{m} \longrightarrow \mathbb{T}^{n}, \quad \iota\left(u_{1}, u_{2}, \ldots, u_{m}\right)= \begin{cases}\left(u_{1}, \bar{u}_{1}, \ldots, u_{m}, \bar{u}_{m}\right), & \text { if } n=2 m ; \\ \left(u_{1}, \bar{u}_{1}, \ldots, u_{m}, \bar{u}_{m}, 1\right), & \text { if } n=2 m+1 ;\end{cases}
$$

induces a $\mathbb{T}^{m}$-action on $\mathbb{P}^{n-1}$ that commutes with the involution $\phi$. Under this embedding,

$$
\left.\left(\alpha_{1}, \ldots, \alpha_{n}\right)\right|_{\mathbb{T}^{m}}= \begin{cases}\left(\lambda_{1},-\lambda_{1}, \ldots, \lambda_{m},-\lambda_{m}\right), & \text { if } n=2 m \\ \left(\lambda_{1},-\lambda_{1}, \ldots, \lambda_{m},-\lambda_{m}, 0\right), & \text { if } n=2 m+1 .\end{cases}
$$

A subspace $Y \subset \mathbb{P}^{n-1}$ is called $\phi$-invariant ( $\mathbb{T}^{m}$-invariant) if $\phi(Y)=Y\left(u(Y)=Y\right.$ for all $\left.u \in \mathbb{T}^{m}\right)$; $Y$ is called $\phi$-fixed ( $\mathbb{T}^{m}$-fixed) if $\phi(y)=y$ for all $y \in Y\left(u(y)=y\right.$ for all $y \in Y$ and $\left.u \in \mathbb{T}^{m}\right)$. The next lemma describes the $\mathbb{T}^{m}$ - and $\phi$-fixed and invariant zero- and one-dimensional subspaces of $\mathbb{P}^{n-1}$. If $n=2 m+1,1 \leqslant i \leqslant m$, and $a, b \in \mathbb{C}^{*}$, then

$$
\mathcal{C}_{i}(a, b) \equiv\left\{\left[z_{1}, \ldots, z_{n}\right] \in \mathbb{P}^{n}: z_{k}=0 \forall k \neq 2 i-1,2 i, n, a z_{2 i-1} z_{2 i}-b z_{n}^{2}=0\right\}
$$

is a smooth conic contained in the plane $\mathbb{P}_{2 i-1,2 i, n}^{2}$ spanned by $P_{2 i-1}, P_{2 i}, P_{n}$ and passing through $P_{2 i-1}$ and $P_{2 i}$.

Lemma 4.1 ([29, Lemma 3.1]). Suppose $n \in \mathbb{Z}^{+}$and $m=\lfloor n / 2\rfloor$. If $n=2 m$, let $\phi$ be either the involution $\tau_{n}^{\prime}$ or $\eta_{2 m}$ on $\mathbb{P}^{n-1}$; if $n=2 m+1$, let $\phi=\tau_{n}^{\prime}$.

(1) The $\mathbb{T}^{m}$-fixed points in $\mathbb{P}^{n-1}$ are the points $P_{i}$ in 4.5$)$ with $i \in[n]$.

(2) If $n=2 m$, the $\mathbb{T}^{m}$-invariant irreducible curves in $\mathbb{P}^{n-1}$ are the lines $\mathbb{P}_{i j}^{1}$ as in (4.6). If $n=2 m+1$, the $\mathbb{T}^{m}$-invariant irreducible curves in $\mathbb{P}^{n-1}$ are the lines $\mathbb{P}_{i j}^{1}$ with $1 \leqslant i \neq j \leqslant n$ and the conics $\mathcal{C}_{i}(a, b)$ as in (4.11) with $a, b \in \mathbb{C}^{*}$. 
(3) If $n=2 m$, the $\phi$-invariant $\mathbb{T}^{m}$-invariant irreducible curves in $\mathbb{P}^{n-1}$ are the lines $\mathbb{P}_{2 i-1,2 i}^{1}$ with $1 \leqslant i \leqslant m$. If $n=2 m+1$, the $\phi$-invariant $\mathbb{T}^{m}$-invariant irreducible curves in $\mathbb{P}^{n-1}$ are the lines $\mathbb{P}_{2 i-1,2 i}^{1}$ with $1 \leqslant i \leqslant m$ and the conics $\mathcal{C}_{i}(a, b)$ with $1 \leqslant i \leqslant m$ and $a, b \in \mathbb{C}^{*}$ such that $a \bar{b} \in \mathbb{R}$.

Remark 4.2. If $n=2 m+1$ and $a, b \in \mathbb{C}^{*}$ are such that $a \bar{b} \in \mathbb{R}$, then

$$
\mathcal{C}_{i}(a, b)^{\phi}= \begin{cases}\left\{\left[z_{1}, \ldots, z_{n}\right] \in \mathbb{P}_{2 i-1,2 i, n}^{2}:\left|z_{2 i-1}\right|=\left|z_{2 i}\right|\right\}, & \text { if } a \bar{b} \in \mathbb{R}^{+} \\ \varnothing, & \text { if } a \bar{b} \in \mathbb{R}^{-} .\end{cases}
$$

\subsection{Equivariant localization data}

Since the $\mathbb{T}^{m}$-action on $\mathbb{P}^{n-1}$ commutes with the involution $\phi$, it induces an action on $\overline{\mathfrak{M}}_{g, l}\left(\mathbb{P}^{n-1}, d\right)^{\phi}$. This action lifts to an action on the total space of the "fibration" (4.1). Theorem 4.6 describes the class (4.3) as a sum of contributions from the fixed loci of the $\mathbb{T}^{m}$-action on $\overline{\mathfrak{M}}_{g, l}\left(\mathbb{P}^{n-1}, d\right)^{\phi}$. Each such contribution is a rational fraction in the weights $\alpha_{1}, \ldots, \alpha_{n}$ of the standard representation of $\mathbb{T}^{n}$ on $\mathbb{C}^{n}$ restricted to the subtorus $\mathbb{T}^{m} \subset \mathbb{T}^{n}$ defined by the embedding (4.9); thus, it is a rational fraction in the weights $\lambda_{1}, \ldots, \lambda_{m}$ of the standard representation of $\mathbb{T}^{m}$ on $\mathbb{C}^{m}$. As in the complex case described in detail in [18, Chapter 27], all such contributions (that do not cancel with other contributions) come from fixed loci corresponding to decorated graphs. In contrast to the complex case, there are also more complicated fixed loci involving covers of the conics (4.11) if $n=2 m+1$. These are dealt with in Section 5, where Theorem 4.6 is justified.

A graph (Ver, Edg) is a pair consisting of a finite set Ver of vertices and an element

$$
\operatorname{Edg} \in \operatorname{Sym}^{k}\left(\left\{\operatorname{Ver}^{\prime} \subset \operatorname{Ver}:\left|\operatorname{Ver}^{\prime}\right|=2\right\}\right)
$$

for some $k \in \mathbb{Z}^{\geqslant 0}$. We will view Edg as a collection of two-element subsets of Ver, called edges, but it may contain several copies of the same two-element subset. However, we do not allow an edge from a vertex back to itself. For $e \in \operatorname{Edg}$ and $v \in e$, let $e / v \in$ Ver denote the vertex in $e$ other than $v$. A graph (Ver, Edg) is connected if for all $v, v^{\prime} \in$ Ver with $v \neq v^{\prime}$ there exist

$$
k \in \mathbb{Z}^{+}, \quad v_{1}, \ldots, v_{k-1} \in \mathrm{Ver}, \quad e_{1}, \ldots, e_{k} \in \mathrm{Edg} \quad \text { s.t. } \quad v_{i-1}, v_{i} \in e_{i} \forall i=1, \ldots, k,
$$

with $v_{0} \equiv v$ and $v_{k} \equiv v^{\prime}$. For any graph (Ver, Edg), let

$$
g(\operatorname{Ver}, \mathrm{Edg}) \equiv|\operatorname{Edg}|-|\operatorname{Ver}|+1
$$

be its genus. An automorphism of a graph (Ver, Edg) is a bijection

$$
h: \text { Ver } \sqcup \text { Edg } \longrightarrow \text { Ver } \sqcup \text { Edg }
$$

such that $h($ Ver $)=$ Ver and $h(v) \in h(e)$ whenever $v \in$ Ver, $e \in \operatorname{Edg}$, and $v \in e$. A subgraph of (Ver, Edg) is a graph $\left(\operatorname{Ver}^{\prime}, \operatorname{Edg}^{\prime}\right)$ such that $\operatorname{Ver}^{\prime} \subset$ Ver and $\operatorname{Edg}^{\prime} \subset \mathrm{Edg}$.

For a finite set $S$, an $S$-marked [n]-labeled decorated graph (or simply decorated graph) is a tuple

$$
\Gamma \equiv(\operatorname{Ver}, \operatorname{Edg}, \mathfrak{g}, \vartheta, \mathfrak{d}, \mathfrak{m})
$$

consisting of a graph (Ver, Edg) and maps

$$
\mathfrak{g}: \text { Ver } \longrightarrow \mathbb{Z}^{\geqslant 0}, \quad \vartheta: \text { Ver } \longrightarrow[n], \quad \mathfrak{d}: \operatorname{Edg} \longrightarrow \mathbb{Z}^{+}, \quad \mathfrak{m}: S \longrightarrow \text { Ver }
$$


such that $\vartheta(v) \neq \vartheta(e / v)$ for every $v \in e$ and $e \in \operatorname{Edg}$. We define the genus $g(\Gamma)$ and the degree $d(\Gamma)$ of such a graph by

$$
g(\Gamma)=g(\operatorname{Ver}, \operatorname{Edg})+\sum_{v \in \operatorname{Ver}} \mathfrak{g}(v) \quad \text { and } \quad d(\Gamma)=\sum_{e \in \operatorname{Edg}} \mathfrak{d}(e),
$$

respectively. For each $v \in$ Ver, let

$$
\mathrm{E}_{v}(\Gamma)=\{e \in \operatorname{Edg}: v \in e\} \quad \text { and } \operatorname{val}_{v}(\Gamma)=2 \mathfrak{g}(v)+\left|\mathrm{E}_{v}(\Gamma)\right|+\left|\mathfrak{m}^{-1}(v)\right|
$$

denote the set of edges leaving the vertex $v$ and its valence.

An automorphism of a decorated graph $\Gamma$ as in (4.12) is an automorphism $h$ of the graph (Ver, Edg) such that

$$
\mathfrak{g}=\left.\mathfrak{g} \circ h\right|_{\text {Ver }}, \quad \vartheta=\left.\vartheta \circ h\right|_{\text {Ver }}, \quad \mathfrak{d}=\left.\mathfrak{d} \circ h\right|_{\text {Edg }}, \quad \mathfrak{m}=h \circ \mathfrak{m} .
$$

A decorated subgraph of a decorated graph $\Gamma$ as in (4.12) is a tuple

$$
\Gamma^{\prime} \equiv\left(\operatorname{Ver}^{\prime}, \operatorname{Edg}^{\prime}, \mathfrak{g}^{\prime}, \vartheta^{\prime}, \mathfrak{d}^{\prime}, \mathfrak{m}^{\prime}\right)
$$

such that $\left(\operatorname{Ver}^{\prime}, \operatorname{Edg}^{\prime}\right)$ is a subgraph of (Ver, Edg),

$$
\left(\mathfrak{g}^{\prime}, \vartheta^{\prime}\right)=\left.(\mathfrak{g}, \vartheta)\right|_{\mathrm{Ver}^{\prime}}, \quad \mathfrak{d}^{\prime}=\left.\mathfrak{d}\right|_{\mathrm{Edg}^{\prime}}, \quad \mathfrak{m}^{\prime}=\left.\mathfrak{m}\right|_{\mathfrak{m}^{-1}\left(\mathrm{Ver}^{\prime}\right)} .
$$

An involution $\sigma$ on a decorated graph $\Gamma$ as in (4.12) is an automorphism of the graph (Ver, Edg) and the set $S$ such that

$$
\sigma \circ \sigma=\mathrm{id}, \quad \mathfrak{g}=\left.\mathfrak{g} \circ \sigma\right|_{\mathrm{Ver}}, \quad \phi \circ \vartheta=\left.\vartheta \circ \sigma\right|_{\text {Ver }}, \quad \mathfrak{d}=\left.\mathfrak{d} \circ \sigma\right|_{\mathrm{Edg}}, \quad \sigma \circ \mathfrak{m}=\left.\mathfrak{m} \circ \sigma\right|_{S},
$$

with $\phi$ as in (2.5). In such a case, let

$$
\mathrm{V}_{\mathbb{R}}^{\sigma}(\Gamma) \subset \text { Ver } \quad \text { and } \quad \mathrm{E}_{\mathbb{R}}^{\sigma}(\Gamma) \subset \operatorname{Edg}
$$

be the subsets consisting of the fixed points of $\sigma$ and define

$$
\mathrm{V}_{\mathbb{C}}^{\sigma}(\Gamma) \equiv \operatorname{Ver}-\mathrm{V}_{\mathbb{R}}^{\sigma}(\Gamma), \quad \mathrm{E}_{\mathbb{C}}^{\sigma}(\Gamma) \equiv \operatorname{Edg}-\mathrm{E}_{\mathbb{R}}^{\sigma}(\Gamma)
$$

If $\mathrm{V}_{\mathbb{R}}^{\sigma}(\Gamma) \neq \varnothing$, then $n=2 m+1$ and $\vartheta(v)=n$ for all $v \in \mathrm{V}_{\mathbb{R}}^{\sigma}(\Gamma)$. If $e \in \mathrm{E}_{\mathbb{R}}^{\sigma}(\Gamma), v_{1}, v_{2} \in e$, and $v_{1} \neq v_{2}$, then $\vartheta\left(v_{1}\right)=\phi\left(\vartheta\left(v_{2}\right)\right)$. If $n=2 m+1$, then $\vartheta(v) \neq n$ for all $v \in e$ with $e \in \mathrm{E}_{\mathbb{R}}^{\sigma}(\Gamma)$. An automorphism of a pair $(\Gamma, \sigma)$ consisting of a decorated graph with an involution is an automorphism $h$ of $\Gamma$ such that $h \circ \sigma=\sigma \circ h$. We denote the group of all automorphisms of $(\Gamma, \sigma)$ by $\operatorname{Aut}(\Gamma, \sigma)$.

The elements of the set $\mathrm{E}_{\mathbb{C}}^{\sigma}(\Gamma)$ above are called Klein edges in [32, Section 3]; the elements of $\mathrm{E}_{\mathbb{R}}^{\sigma}(\Gamma)$ are doubled half-edges or disk edges in the terminology of [32]. A graph with an involution can be depicted as in Figure 1. The label next to each edge $e$ indicates the value of $\mathfrak{d}$ on $e$. Each vertex $v$ should similarly be labeled by the pair $(\mathfrak{g}(v), \vartheta(v))$; we drop the first label if it is zero. The involution $\sigma$ is indicated by the two-sided arrows in Figure 1. For example, it exchanges the two vertices in the first two diagrams. It flips each of the edges back to itself in the first diagram, 

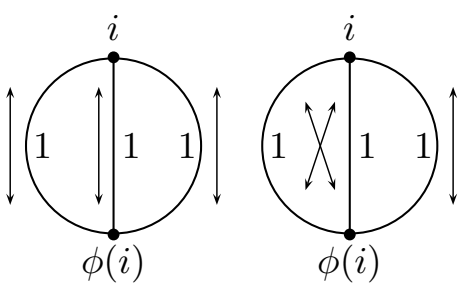
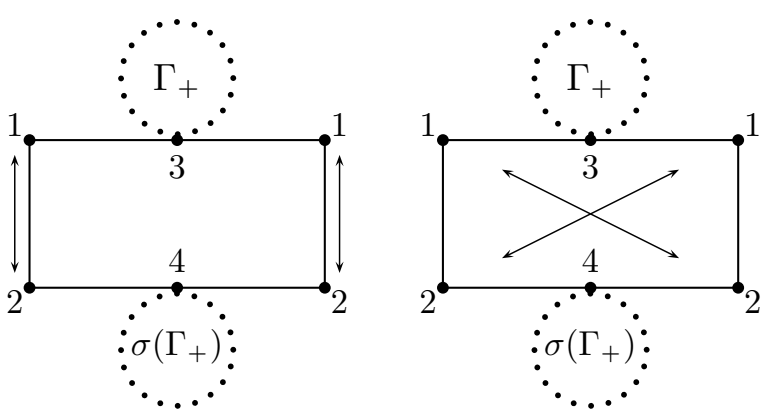

Figure 1: Examples of decorated graphs with involutions.

but exchanges two of them in the second. The first diagram contains no Klein edges, while the second contains a pair of such edges. In the first two diagrams, $S=\varnothing$. In the last two diagrams, the disks indicate any possibility for the graph $\Gamma_{+}$.

We will call a pair $(\Gamma, \sigma)$ consisting of a connected decorated graph with an involution admissible if $\Gamma$ is a connected graph,

$$
\mathfrak{d}(e) \notin 2 \mathbb{Z} \quad \forall v \in \mathrm{E}_{\mathbb{R}}^{\sigma}(\Gamma) \quad \text { and } \quad \vartheta(v) \neq 2 m+1 \quad \forall v \in \mathrm{Edg} .
$$

The second condition in (4.15), which is relevant only if $n \notin 2 \mathbb{Z}$, implies that $\mathrm{V}_{\mathbb{R}}^{\sigma}(\Gamma)=\varnothing$. For $d, g, l \in \mathbb{Z}^{\geqslant 0}$, let $\mathcal{A}_{g, l}(n, d)$ denote the set of admissible pairs $(\Gamma, \sigma)$ such that $\Gamma$ is an $S_{l}$-marked $[n]$-labeled decorated graph with $g(\Gamma)=g, d(\Gamma)=d$,

$$
S_{l} \equiv\left\{1^{+}, 1^{-}, \ldots, l^{+}, l^{-}\right\}, \quad \sigma\left(i^{ \pm}\right)=i^{\mp} \quad \forall i=1, \ldots, l .
$$

We show in Section 5 that all equivariant contributions to (4.3) arise from the fixed loci corresponding to the elements of $\mathcal{A}_{g, l}(n, d)$.

In the remainder of this section, we describe the equivariant contributions arising from the elements of $\mathcal{A}_{g, l}(n, d)$. Fix tuples

$$
\mathbf{b} \equiv\left(b_{1}, \ldots, b_{l}\right) \in\left(\mathbb{Z}^{\geqslant 0}\right)^{l} \quad \text { and } \quad \mathbf{p} \equiv\left(p_{1}, \ldots, p_{l}\right) \in\left(\mathbb{Z}^{\geqslant 0}\right)^{l} .
$$

We will describe the equivariant localization contributions to (4.4) under the assumptions that $n-k \in 2 \mathbb{Z}$ and either

- $\phi=\tau_{n}^{\prime}$ and $(n, \mathbf{a})$ satisfies the conditions of Proposition 1.4(1) or

- $\phi=\eta_{2 m}$ and $(n, \mathbf{a})$ satisfies the conditions of Proposition 1.4(2) with $n=2 m$.

If $k=0$, i.e. $X_{n ; \mathbf{a}}=\mathbb{P}^{n-1}$, the equivariant Euler class in (4.4) is 1 . If $g=0$, this class is welldefined over the entire moduli space as an element in the cohomology of $\overline{\mathfrak{M}}_{0, l}\left(\mathbb{P}^{n-1}, d\right)^{\phi}$ twisted by the orientation system of this moduli space. The integral in (4.4) then computes the genus 0 real GW-invariants of $\left(X_{n ; \mathbf{a}}, \omega_{n ; \mathbf{a}}, \phi_{n ; \mathbf{a}}\right)$. In other cases, this Euler class is well-defined over the subspace (4.2). For the admissible pairs $(\Gamma, \sigma)$ with $\mathfrak{g}(v)=0$ for all $v \in$ Ver, Theorem 4.6 below then describes the contribution of the $\mathbb{T}^{m}$-fixed locus in $\overline{\mathfrak{M}}_{g, l}\left(\mathbb{P}^{n-1}, d\right)^{\phi}$ corresponding to ( $\left.\Gamma, \sigma\right)$ to the integral in (4.4) of any extension of $\mathbf{e}\left(\mathcal{V}_{n ; \mathbf{a}}^{\widetilde{\phi}_{n ; \mathbf{a}}}\right)$ to an equivariant cohomology class over the entire 
space. In the case $g=1$ and $\operatorname{dim}_{\mathbb{C}} X_{n ; \mathbf{a}}=3$, it is expected that this class integral is independent of the extension and computes the genus 1 real GW-invariants of $\left(X_{n ; \mathbf{a}}, \omega_{n ; \mathbf{a}}, \phi_{n ; \mathbf{a}}\right)$.

For $\mathbf{a} \in\left(\mathbb{Z}^{+}\right)^{k}$ as before, let

$$
\langle\mathbf{a}\rangle=a_{1} \cdot \ldots \cdot a_{k} .
$$

For $g \in \mathbb{Z}^{\geqslant 0}$ and a finite set $S$ with $2 g+|S| \geqslant 3$, denote by $\overline{\mathcal{M}}_{g, S}$ the usual Deligne-Mumford moduli space of stable genus $g S$-marked curves and by

$$
\mathbb{E} \longrightarrow \overline{\mathcal{M}}_{g, S}
$$

the Hodge vector bundle of holomorphic differentials. For each $i \in S$, let

$$
\psi_{i} \in H^{2}\left(\overline{\mathcal{M}}_{g, S} ; \mathbb{Q}\right)
$$

be the first Chern class of the universal cotangent line bundle associated with the $i$-th marked point.

Suppose $(\Gamma, \sigma)$ is an element of $\mathcal{A}_{g, l}(n, d)$ with $\Gamma$ as in (4.12). For each $v \in$ Ver, let

$$
\begin{gathered}
S_{v}=\mathrm{E}_{v}(\Gamma) \sqcup \mathfrak{m}^{-1}(v), \quad S_{v}^{-}=\left\{i=1, \ldots, l: i^{-} \in S_{v}\right\}, \quad|\mathbf{b}|_{v}=\sum_{\substack{1 \leqslant i \leqslant l \\
i \pm \in S_{v}}} b_{i}, \quad|\mathbf{p}|_{v}=\sum_{\substack{1 \leqslant i \leqslant l \\
i \pm \in S_{v}}} p_{i}, \\
\mathfrak{s}_{v}=\mathfrak{g}(v)-1+\left|\mathrm{E}_{v}(\Gamma)\right|+\sum_{i \in S_{v}^{-}}\left(1+b_{i}+p_{i}\right), \quad \psi_{e ; v}=\frac{\alpha_{\vartheta(e / v)}-\alpha_{\vartheta}(v)}{\mathfrak{d}(e)} \forall e \in \mathrm{E}_{v}(\Gamma) .
\end{gathered}
$$

If $\operatorname{val}_{v}(\Gamma) \geqslant 3$, let

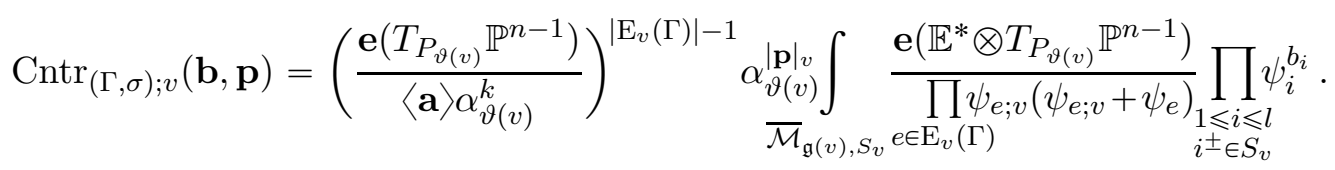

If $\operatorname{val}_{v}(\Gamma)=1,2$, let

$$
\begin{aligned}
\operatorname{Cntr}_{(\Gamma, \sigma) ; v}(\mathbf{b}, \mathbf{p})=-(-1)^{\operatorname{val}_{v}(\Gamma)} & \left(\frac{\mathbf{e}\left(T_{P_{\vartheta(v)}} \mathbb{P}^{n-1}\right)}{\langle\mathbf{a}\rangle \alpha_{\vartheta(v)}^{k}}\right)^{\left|\mathrm{E}_{v}(\Gamma)\right|-1} \alpha_{\vartheta(v)}^{|\mathbf{p}|_{v}} \\
& \times\left(\prod_{e \in \mathrm{E}_{v}(\Gamma)} \psi_{e ; v}\right)^{-1}\left(\sum_{e \in \mathrm{E}_{v}(\Gamma)} \psi_{e ; v}\right)^{3-\operatorname{val}_{v}(\Gamma)-\left|\mathrm{E}_{v}(\Gamma)\right|+|\mathbf{b}|_{v}} .
\end{aligned}
$$

In light of (4.7), the equivariant Euler classes of $T_{P_{\vartheta(v)}} \mathbb{P}^{n-1}$ and $\mathbb{E}^{*} \otimes T_{P_{\vartheta}(v)} \mathbb{P}^{n-1}$ are readily expressible in terms of the torus weights $\alpha_{1}, \ldots, \alpha_{n}$ and the Hodge classes $c_{i}(\mathbb{E})$ on $\overline{\mathcal{M}}_{\mathfrak{g}(v), S_{v}}$.

Remark 4.3. Our vertex contributions, i.e. (4.18) and (4.19), include the movements of the nodes associated with the edges $e \in \mathrm{E}_{v}(\Gamma)$, in contrast to [18, (27.8)] and [32, (3.22)]. The right-hand sides of these equations are the standard vertex contributions in the complex setting. The inclusion of the node movements has the effect of dividing the product of all factors on the first two lines in [18, (27.8)] and the last two lines in [32, (3.22)] associated with $v$ by the product of $-\psi_{e ; v}$ with $e \in \mathrm{E}_{v}(\Gamma)$. In the real setting, each vertex contribution comes with the sign $(-1)^{\mathfrak{s}_{v}} ;$ see (4.26). The contribution from $S_{v}^{-}$comes from orienting the moduli space by the positive marked points. The 
contributions of $\mathfrak{g}(v)-1$ and $\left|\mathrm{E}_{v}(\Gamma)\right|$ appear for more delicate reasons. The first arises from the comparison between the orientation on the moduli space (5.8) of real maps from the nodal doublets (5.7) induced by a real orientation and the standard complex orientation on the moduli space of maps from one of the components; see the first equation in (5.30) and [12, Theorem 1.4]. The second sign contribution arises because the complex line bundle of smoothings of the node associated with each flag $(e, v)$ should be taken with the anti-complex orientation; see (5.24) and [12, Theorem 1.2]. The product of these extra signs over all relevant vertices constitutes the leading sign in [32, (3.15)].

With $(\Gamma, \sigma)$ as in the previous paragraph, let $e \in \operatorname{Edg}$ and $v_{1}, v_{2} \in e$ be the two vertices of $e$. Suppose first that $e \in \mathrm{E}_{\mathbb{C}}^{\sigma}(\Gamma)$. If

$$
n=2 m+1, \quad \mathfrak{d}(e) \in 2 \mathbb{Z}, \quad \text { and } \quad \vartheta\left(v_{1}\right)=\phi\left(\vartheta\left(v_{2}\right)\right)
$$

then $k \geqslant 1$. In this case, we set $\operatorname{Cntr}_{(\Gamma, \sigma) ; e}=0$ if $k \geqslant 2$ and

$$
\operatorname{Cntr}_{(\Gamma, \sigma) ; e}=\frac{(-1)^{\left(a_{1}+1\right) \mathfrak{d}(e) / 2}}{\mathfrak{d}(e)} \frac{a_{1}\left(\left(a_{1} \mathfrak{d}(e) / 2\right) !\right)^{2}}{((\mathfrak{d}(e) / 2) !)^{2}(\mathfrak{d}(e) !)^{2}} \frac{\left(\frac{2 \alpha_{\vartheta(v)}}{\mathfrak{d}(e)}\right)^{\left(a_{1}-3\right) \mathfrak{d}(e)+2}}{\prod_{\substack{1 \leqslant j<n \\ j \neq \vartheta\left(v_{1}\right), \vartheta\left(v_{2}\right)}}\left(\alpha_{j} \prod_{r=1}^{\mathfrak{d}(e) / 2}\left(r^{2}\left(\frac{2 \alpha_{\vartheta}(v)}{\mathfrak{d}(e)}\right)^{2}-\alpha_{j}^{2}\right)\right)}
$$

if $k=1$ and $v \in e$ is either vertex. If one of the conditions in (4.20) is not satisfied, then let

$$
\operatorname{Cntr}_{(\Gamma, \sigma) ; e}=\frac{(-1)^{\mathfrak{d}(e)}}{\mathfrak{d}(e)(\mathfrak{d}(e) !)^{2}} \frac{\prod_{i=1}^{k} \prod_{r=0}^{a_{i} \mathfrak{d}(e)} \frac{\left(a_{i} \mathfrak{d}(e)-r\right) \alpha_{\vartheta\left(v_{1}\right)}+r \alpha_{\vartheta}\left(v_{2}\right)}{\mathfrak{d}(e)}}{\left(\frac{\alpha_{\vartheta\left(v_{1}\right)}-\alpha_{\vartheta\left(v_{2}\right)}}{\mathfrak{d}(e)}\right)^{2 \mathfrak{d}(e)-2} \prod_{j \neq \vartheta\left(v_{1}\right), \vartheta\left(v_{2}\right)} \prod_{r=0}^{\mathfrak{d}(e)}\left(\frac{(\mathfrak{d}(e)-r) \alpha_{\vartheta\left(v_{1}\right)}+r \alpha_{\vartheta\left(v_{2}\right)}}{\mathfrak{d}(e)}-\alpha_{j}\right)}
$$

If $e \in \mathrm{E}_{\mathbb{R}}^{\sigma}(\Gamma)$, then $\mathfrak{d}(e) \notin 2 \mathbb{Z}$ by the first assumption in (4.15). If in addition $a_{i} \notin 2 \mathbb{Z}$ for all $i=1, \ldots, k$, let

$$
\operatorname{Cntr}_{(\Gamma, \sigma) ; e}=\frac{(-1)^{|\phi|+\frac{\mathfrak{d}(e)-1}{2}}}{\mathfrak{d}(e)} \frac{\prod_{i=1}^{k}\left(a_{i} \mathfrak{d}(e)\right) ! !}{2^{\mathfrak{d}(e)-1} \mathfrak{d}(e) !} \frac{\left(\frac{\alpha_{\vartheta\left(v_{1}\right)}}{\mathfrak{d}(e)}\right)^{\frac{(|\mathbf{a}|-2) \mathfrak{d}(e)+k}{2}+1}}{\prod_{j \neq \vartheta\left(v_{1}\right), \vartheta\left(v_{2}\right)} \prod_{r=0}^{(\mathfrak{d}(e)-1) / 2}\left(\frac{(\mathfrak{d}(e)-2 r) \alpha_{\vartheta}\left(v_{1}\right)}{\mathfrak{d}(e)}-\alpha_{j}\right)} .
$$

If $a_{i} \in 2 \mathbb{Z}$ for some $i=1, \ldots, k$, set $\operatorname{Cntr}_{(\Gamma, \sigma) ; e}=0$.

Remark 4.4. Our edge contributions, i.e. (4.21), (4.22), and (4.23), do not include the movements of the nodes associated with the vertices $v_{1}, v_{2} \in e$, in contrast to [18, (27.8)] and [32, (3.22)]. They are included into the vertex contributions. The right-hand side in (4.22) is the negative of the standard edge contribution in the complex setting with the automorphism group taken into account. This has the effect of multiplying the product of all factors on the last line in [18, (27.8)] and the first line in [32, (3.22)] associated with $e$ by $-\psi_{e ; v_{1}} \psi_{e ; v_{2}} / \mathfrak{d}(e)$. The extra negative sign for each Klein edge arises due to [12, Theorem 1.4]; see Remark 4.3 and the second equation in (5.30). The product of these extra signs over all relevant Klein edges constitutes the leading sign in [32, $(3.22)]$. For $(n, \mathbf{a})=(5,(5))$ and $(n, k)=(2 m, 0)$ with $m \in 2 \mathbb{Z}$, (4.23) becomes the disk factor on the 
first line in [32, (3.22)] multiplied by $-\psi_{e ; v_{1}} / \mathfrak{d}(e)$ and [3, (6.21)] multiplied by $-(-1)^{|\phi|} \psi_{e ; v_{1}} / \mathfrak{d}(e)$, respectively. For $(n, k)=(2 m, 0)$ with $m \notin 2 \mathbb{Z}$ and $\phi=\tau_{n}^{\prime}$, (4.23) becomes [3, (6.21)] multiplied by

$$
-(-1)^{(\mathfrak{d}(e)-1) / 2} \psi_{e ; v_{1}} / \mathfrak{d}(e) .
$$

Along with the node sign correction of [3, Remark 6.9], the extra factor of $(-1)^{(\mathfrak{d}(e)-1) / 2}$ accounts for the difference between our canonical orientation on the moduli space and that induced by the relative spin structure of [3, Remark 6.5]; see [12, Corollary 3.6] and the second case in Section 5.4. The map automorphisms arising from the edge degrees are absorbed into the automorphism factor [32, (3.15)]; these automorphisms and the factor of $(-1)^{|\phi|}$ arising from the $\eta$ involution on the domain is taken into account in [3, (6.7)].

Remark 4.5. The right-hand side in (4.21) is obtained from the right-hand side in (4.22) by first setting $\alpha_{n}=0$ as required by (4.10), simplifying the resulting expression, and then setting $\alpha_{\vartheta\left(v_{1}\right)}=-\alpha_{\vartheta\left(v_{2}\right)}$. This corresponds to resolving the $\frac{0}{0}$ ambiguity described below [32, (3.23)] "from" the left diagram in [32, Figure 5]. The restrictions of the denominators in (4.22) may vanish because some fixed loci of the $\mathbb{T}^{m}$-action on $\overline{\mathfrak{M}}_{g, l}\left(\mathbb{P}^{n-1}, d\right)^{\phi}$ consist of covers of the conics $\mathcal{C}_{i}(a, b)$ of Lemma 4.1](3), see Section 5.1. The equivariant contributions from these fixed loci are computed in Lemma 5.4 using an auxiliary $S^{1}$-action. The right diagram in [32, Figure 5] involves graphs $\Gamma$ with vertices $v \in$ Ver mapped by $\vartheta$ to $2 m+1$; the second condition in (4.15) excludes such graphs in our perspective.

Since $\mathrm{V}_{\mathbb{R}}^{\sigma}(\Gamma)=\varnothing$, we can choose a subset $\mathrm{V}_{+}^{\sigma}(\Gamma)$ of Ver such that

$$
\text { Ver }=\mathrm{V}_{+}^{\sigma}(\Gamma) \sqcup \mathrm{V}_{-}^{\sigma}(\Gamma) \quad \text { with } \quad \mathrm{V}_{-}^{\sigma}(\Gamma) \equiv \sigma\left(\mathrm{V}_{+}^{\sigma}(\Gamma)\right)
$$

We can also choose a subset $\mathrm{E}_{+}^{\sigma}(\Gamma)$ of $\mathrm{E}_{\mathbb{C}}^{\sigma}(\Gamma)$ such that

$$
\operatorname{Edg}=\mathrm{E}_{\mathbb{R}}^{\sigma}(\Gamma) \sqcup \mathrm{E}_{+}^{\sigma}(\Gamma) \sqcup \mathrm{E}_{-}^{\sigma}(\Gamma) \quad \text { with } \quad \mathrm{E}_{-}^{\sigma}(\Gamma) \equiv \sigma\left(\mathrm{E}_{+}^{\sigma}(\Gamma)\right) .
$$

Theorem 4.6. Suppose $(\Gamma, \sigma) \in \mathcal{A}_{g, l}(n, d)$ with $\Gamma$ as in 4.12). If $\mathfrak{g}(v)=0$ for all $v \in$ Ver or $k=0$, then the contribution of the $\mathbb{T}^{m}$-fixed locus in $\overline{\mathfrak{M}}_{g, l}\left(\mathbb{P}^{n-1}, d\right)^{\phi}$ corresponding to $(\Gamma, \sigma)$ to 4.4$)$ is the restriction of

$$
\operatorname{Cntr}_{(\Gamma, \sigma)}(\mathbf{b}, \mathbf{p})=\frac{1}{|\operatorname{Aut}(\Gamma, \sigma)|} \prod_{v \in \mathrm{V}_{+}^{\sigma}(\Gamma)}(-1)^{\mathfrak{s}_{v}} \operatorname{Cntr}_{(\Gamma, \sigma) ; v}(\mathbf{b}, \mathbf{p}) \prod_{e \in \mathrm{E}_{\mathbb{R}}^{\sigma}(\Gamma) \sqcup \mathrm{E}_{+}^{\sigma}(\Gamma)} \operatorname{Cntr}_{(\Gamma, \sigma) ; e}
$$

to the subtorus $\mathbb{T}^{m} \subset \mathbb{T}^{n}$ as in (4.10). The integral in (4.4) is the sum of these contributions over $\mathcal{A}_{g, l}(n, d)$ if $g=0,1$ or $k=0$.

Remark 4.7. As indicated in Section 5.3, the transfer of the movement of the node corresponding to a flag $(e, v)$ from the contribution for $e$ to the contribution for $v$ in effect marks all nodes with $v \in \mathrm{V}_{+}^{\sigma}(\Gamma)$ as positive. This is used to compare the orientation associated with $(\Gamma, \sigma)$ to the orientations associated with its edges and vertices. This transfer allows us to choose the subsets $\mathrm{V}_{+}^{\sigma}(\Gamma)$ and $\mathrm{E}_{+}^{\sigma}(\Gamma)$ at random, as long as the conditions (4.24) and (4.25) are satisfied. We show directly in Section 4.3 that (4.26) is independent of all choices made, provided the conditions of Proposition 1.4 and Theorem 4.6 are satisfied and $n-k \in 2 \mathbb{Z}$ (otherwise, the moduli space of real maps into $\left(X_{n ; \mathbf{a}}, \phi_{n ; \mathbf{a}}\right)$ may not be orientable). 


\subsection{Examples and applications}

We now make a number of observations regarding the contributions of Theorem 4.6, apply it in some specific cases, give an alternative proof of Theorem 1.6](2) in the case of projective spaces, and establish Proposition 1.7.

If the restrictions of Proposition 1.4 and Theorem 4.6 are satisfied and $n-k \in 2 \mathbb{Z}$, the contribution (4.26) from an admissible graph $(\Gamma, \sigma)$ is independent of all choices made:

$$
v_{1} \in e \quad \forall e \in \operatorname{Edg}, \quad \mathrm{V}_{+}^{\sigma}(\Gamma) \subset \mathrm{V}_{\mathbb{C}}^{\sigma}(\Gamma), \quad \text { and } \quad \mathrm{E}_{+}^{\sigma}(\Gamma) \subset \mathrm{E}_{\mathbb{C}}^{\sigma}(\Gamma) .
$$

The right-hand sides of (4.21) and (4.22) are symmetric in $v_{1}$ and $v_{2}$, even before restricting to the subtorus $\mathbb{T}^{m} \subset \mathbb{T}^{n}$. Suppose $e \in \mathrm{E}_{\mathbb{R}}^{\sigma}(\Gamma)$ and $v_{1}, v_{2} \in e$. Since $\mathfrak{d}(e) \notin 2 \mathbb{Z}$ and $\alpha_{\vartheta\left(v_{1}\right)}=-\alpha_{\vartheta\left(v_{2}\right)}$ on $\mathbb{T}^{m}$ in this case, replacing $v_{1}$ by $v_{2}$ in (4.23) changes the restriction of its right-hand side to $\mathbb{T}^{m}$ by the factor of -1 to the power of

$$
\frac{(|\mathbf{a}|-2) \mathfrak{d}(e)+k}{2}+1+\frac{\mathfrak{d}(e)+1}{2}(n-2) \equiv \frac{1}{2}((n+|\mathbf{a}|) \mathfrak{d}(e)+(n+k)) \quad \bmod 2 .
$$

If $a_{i} \notin 2 \mathbb{Z}$ for all $i=1, \ldots, k$, then Lemma 2.2 implies that

$$
\left.\frac{1}{2}((n+|\mathbf{a}|) \mathfrak{d}(e)+(n+k)) \equiv \frac{1}{2}(n+k)(\mathfrak{d}(e)+1)\right) \quad \bmod 2 .
$$

If $n-k \in 2 \mathbb{Z}$ and $a_{i} \notin 2 \mathbb{Z}$ for all $i=1, \ldots, k$, interchanging $v_{1}$ and $v_{2}$ thus has no effect on the restriction of the right-hand side of (4.23) to $\mathbb{T}^{m}$. If $a_{i} \in 2 \mathbb{Z}$ for some $i=1, \ldots, k$, then $\operatorname{Cntr}_{(\Gamma, \sigma) ; e}$ is 0. In summary, the edge contributions in (4.26) are independent of the ordering of the vertices of each edge $e$ in $\mathrm{E}_{\mathbb{R}}^{\sigma}(\Gamma) \sqcup \mathrm{E}_{+}^{\sigma}(\Gamma)$.

Replacing $e \in \mathrm{E}_{\mathbb{C}}^{\sigma}(\Gamma)$ by $\sigma(e)$ in (4.22) changes the restriction of its right-hand side to $\mathbb{T}^{m}$ by the factor of -1 to the power of

$$
\sum_{i=1}^{k}\left(a_{i} \mathfrak{d}(e)+1\right)+(2 \mathfrak{d}(e)-2)+(\mathfrak{d}(e)+1)(n-2) \equiv(n+|\mathbf{a}|) \mathfrak{d}(e)+(n+k) \quad \bmod 2 .
$$

If $n-k \in 2 \mathbb{Z}$ and the assumptions of Proposition 1.4 are satisfied, both numbers on the right-hand side above are even. Thus, replacing an element $e \in \mathrm{E}_{+}^{\sigma}(\Gamma)$ with $\sigma(e)$ has no effect on the restrictions of (4.22) and (4.26) to $\mathbb{T}^{m}$. By the first sentence in Remark 4.5, the same is the case of (4.21). In fact, the last conclusion follows from the independence of (4.21) of the choice of $v \in e$, since replacing an element $e \in \mathrm{E}_{+}^{\sigma}(\Gamma)$ with $\sigma(e)$ does not change the subset $\left\{\vartheta\left(v_{1}\right), \vartheta\left(v_{2}\right)\right\}$ of $[n]$ in this case.

Replacing $v \in$ Ver by $\sigma(v)$ changes $(-1)^{\mathfrak{s}_{v}}$ by the factor of -1 to the power of

$$
\left|S_{v}^{-}\right|+\left|S_{\sigma(v)}^{-}\right|+\sum_{\substack{1 \leqslant i \leqslant l \\ i \pm \in S_{v}}} b_{i}+\sum_{\substack{1 \leq i \leq l \\ i \pm \in S_{v}}} p_{i}=\left|\mathfrak{m}^{-1}(v)\right|+|\mathbf{b}|_{v}+|\mathbf{p}|_{v} .
$$

This replacement changes the restriction of the right-hand side of (4.19) to $\mathbb{T}^{m}$ by the factor of -1 to the power of

$$
\begin{aligned}
(n-1-k)\left(\left|\mathrm{E}_{v}(\Gamma)\right|-1\right) & +|\mathbf{b}|_{v}+|\mathbf{p}|_{v}+\left(3-\operatorname{val}_{v}(\Gamma)-2\left|\mathrm{E}_{v}(\Gamma)\right|\right) \\
& \equiv\left|\mathfrak{m}^{-1}(v)\right|+|\mathbf{b}|_{v}+|\mathbf{p}|_{v}+(n-k)\left(\left|\mathrm{E}_{v}(\Gamma)\right|-1\right) \quad \bmod 2 .
\end{aligned}
$$


It changes the restriction of the right-hand side of (4.18) to $\mathbb{T}^{m}$ by the factor of -1 to the power of

$$
\begin{aligned}
(n-1-k)\left(\left|\mathrm{E}_{v}(\Gamma)\right|-1\right) & +|\mathbf{b}|_{v}+|\mathbf{p}|_{v}+(n-1) \mathfrak{g}(v)-2\left|\mathrm{E}_{v}(\Gamma)\right|-\left(3 \mathfrak{g}(v)-3+\left|S_{v}\right|\right) \\
& \equiv\left|\mathfrak{m}^{-1}(v)\right|+|\mathbf{b}|_{v}+|\mathbf{p}|_{v}+(n-k)\left(\left|\mathrm{E}_{v}(\Gamma)\right|-1\right)+n \mathfrak{g}(v) \quad \bmod 2 .
\end{aligned}
$$

If $n-k \in 2 \mathbb{Z}$ and the conditions of Theorem 4.6 are satisfied, the right-hand sides of (4.29) and (4.30) reduce to the right-hand side of (4.28). Thus, replacing an element $v \in \mathrm{V}_{+}^{\sigma}(\Gamma)$ with $\sigma(v)$ has no effect on the restriction of (4.26) to $\mathbb{T}^{m}$.

We next observe that the contributions (4.26) for certain admissible pairs $\left(\Gamma, \sigma_{1}\right)$ and $\left(\Gamma, \sigma_{2}\right)$ are opposites of each other if all automorphisms are ignored. Two examples of such pairs appear in Figure 1. The cardinalities of $\operatorname{Aut}\left(\Gamma, \sigma_{1}\right)$ and $\operatorname{Aut}\left(\Gamma, \sigma_{2}\right)$ are different for the first pair (6 and 2, respectively) and the same for the second pair $\left(2\left|\operatorname{Aut}\left(\Gamma_{+}\right)\right|\right)$.

Corollary 4.8. Suppose $n \in \mathbb{Z}^{+}, g, d, k, l \in \mathbb{Z} \geqslant 0$ with $n-k \in 2 \mathbb{Z}, \mathbf{a} \in\left(\mathbb{Z}^{+}-2 \mathbb{Z}\right)^{k}$, and $\phi=\tau_{n}^{\prime}$ or $n \in 2 \mathbb{Z}$ and $\phi=\eta_{n}$. If $\phi=\tau_{n}^{\prime}$, assume that ( $\left.n, \mathbf{a}\right)$ satisfies the assumptions of Proposition 1.4 (1); if $\phi=\eta_{n}$, assume that $(m \equiv n / 2, \mathbf{a})$ satisfies the assumptions of Proposition 1.4(2). If $\left(\Gamma, \sigma_{1}\right)$ and $\left(\Gamma, \sigma_{2}\right)$ are elements of $\mathcal{A}_{g, l}(n, d)$ such that $k \mathfrak{g}(v)=0$ for all vertices $v$ of $\Gamma$,

$$
\mathrm{E}_{\mathbb{R}}^{\sigma_{1}}(\Gamma) \supset \mathrm{E}_{\mathbb{R}}^{\sigma_{2}}(\Gamma), \quad \text { and } \quad\left|\mathrm{E}_{\mathbb{R}}^{\sigma_{1}}(\Gamma)-\mathrm{E}_{\mathbb{R}}^{\sigma_{2}}(\Gamma)\right|=2,
$$

then

$$
\mathfrak{d}\left(e^{*}\right)\left|\operatorname{Aut}\left(\Gamma, \sigma_{1}\right)\right| \operatorname{Cntr}_{\left(\Gamma, \sigma_{1}\right)}(\mathbf{b}, \mathbf{p})=-\left|\operatorname{Aut}\left(\Gamma, \sigma_{2}\right)\right| \operatorname{Cntr}_{\left(\Gamma, \sigma_{2}\right)}(\mathbf{b}, \mathbf{p}),
$$

where $e^{*}$ is one of the elements of $\mathrm{E}_{\mathbb{R}}^{\sigma_{1}}(\Gamma)-\mathrm{E}_{\mathbb{R}}^{\sigma_{2}}(\Gamma)$.

Lemma 4.9. Let $S$ be a finite set with involutions $\sigma_{1}$ and $\sigma_{2}$ and

$$
S_{\mathbb{R}}^{\sigma_{i}} \equiv\left\{s \in S: \sigma_{i}(s)=s\right\} .
$$

If $S_{\mathbb{R}}^{\sigma_{2}}=\varnothing$, then there exists a subset $S_{+} \subset S$ such that

$$
\left|S_{+} \cap S_{\mathbb{R}}^{\sigma_{1}}\right|=\frac{1}{2}\left|S_{\mathbb{R}}^{\sigma_{1}}\right|, \quad S=S_{\mathbb{R}}^{\sigma_{1}} \sqcup\left(S_{+}-S_{\mathbb{R}}^{\sigma_{1}}\right) \sqcup \sigma_{1}\left(S_{+}-S_{\mathbb{R}}^{\sigma_{1}}\right)=S_{+} \sqcup \sigma_{2}\left(S_{+}\right) .
$$

Proof. Since $\sigma_{2}$ acts without fixed points, the cardinalities of $S$ and $S_{\mathbb{R}}^{\sigma_{1}}$ are even. Let $\sigma_{1}^{\prime}$ be any involution on $S$ such that

$$
S_{\mathbb{R}}^{\sigma_{1}^{\prime}}=\varnothing \quad \text { and }\left.\quad \sigma_{1}^{\prime}\right|_{S-S_{\mathbb{R}}^{\sigma_{1}}}=\left.\sigma_{1}\right|_{S-S_{\mathbb{R}}^{\sigma_{1}}}
$$

In particular, $\sigma_{1}^{\prime}$ restricts to an involution on $S_{\mathbb{R}}^{\sigma_{1}}$ without fixed points. Therefore, a subset $S_{+} \subset S$ that satisfies (4.32) with $\sigma_{1}$ replaced by $\sigma_{1}^{\prime}$ also satisfies (4.32) itself. Thus, it is sufficient to establish the claim under the assumption that $S_{\mathbb{R}}^{\sigma_{1}}=\varnothing$.

Suppose we have constructed a subset $S_{+}^{\prime} \subset S$ such that

$$
\left.S_{+}^{\prime} \cap \sigma_{1}\left(S_{+}^{\prime}\right), S_{+}^{\prime} \cap \sigma_{2}\left(S_{+}^{\prime}\right)=\varnothing, \quad \mid \sigma_{2}\left(S_{+}^{\prime}\right)-S_{+}^{\prime} \cup \sigma_{1}\left(S_{+}^{\prime}\right)\right) \mid \leqslant 1 .
$$

If $S=S_{+}^{\prime} \cup \sigma_{1}\left(S_{+}^{\prime}\right)$, then we can take $S_{+}=S_{+}^{\prime}$. If

$$
S \supsetneq S_{+}^{\prime} \cup \sigma_{1}\left(S_{+}^{\prime}\right) \quad \text { and } \quad \sigma_{2}\left(S_{+}^{\prime}\right) \subset S_{+}^{\prime} \cup \sigma_{1}\left(S_{+}^{\prime}\right) \text {, }
$$


enlarge $S_{+}^{\prime}$ by adding any element from the complement of $S_{+}^{\prime} \cup \sigma_{1}\left(S_{+}^{\prime}\right)$ in $S$. If $\sigma_{2}\left(S_{+}^{\prime}\right)$ is not contained in $S_{+}^{\prime} \cup \sigma_{1}\left(S_{+}^{\prime}\right)$ and $s$ is the unique element in the complement, enlarge $S_{+}^{\prime}$ by adding $\sigma_{1}(s)$ to it; this element is not in $\sigma_{2}\left(S_{+}^{\prime}\right)$ by the uniqueness of $s$. After repeating this procedure finitely many times, we obtain a subset $S_{+} \subset S$ satisfying (4.32).

Proof of Corollary 4.8. Let $\Gamma$ be as in (4.12) and $e_{1}^{*}$ and $e_{2}^{*}$ be the two edges in the complement of $\mathrm{E}_{\mathbb{R}}^{\sigma_{2}}(\Gamma)$ in $\mathrm{E}_{\mathbb{R}}^{\sigma_{1}}(\Gamma)$. We denote the vertices of $e_{1}^{*}$ by $v_{11}^{*}$ and $v_{12}^{*}$ and the vertices of $e_{2}^{*}$ by $v_{21}^{*}$ and $v_{22}^{*}$. Since $\left(\Gamma, \sigma_{1}\right)$ is an admissible graph, $\mathfrak{d}\left(e_{1}^{*}\right), \mathfrak{d}\left(e_{2}^{*}\right) \notin 2 \mathbb{Z}$. The involution $\sigma_{1}$ pairs up the decorated one-edge subgraphs of $\Gamma$ determined by the edges in $\mathrm{E}_{\mathbb{C}}^{\sigma_{1}}(\Gamma)$ and $\sigma_{2}$ pairs up the decorated one-edge subgraphs determined by the edges in

$$
\mathrm{E}_{\mathbb{C}}^{\sigma_{2}}(\Gamma)=\mathrm{E}_{\mathbb{C}}^{\sigma_{1}}(\Gamma) \sqcup\left\{e_{1}^{*}, e_{2}^{*}\right\} .
$$

Thus,

$$
\mathfrak{d}\left(e_{1}^{*}\right)=\mathfrak{d}\left(e_{2}^{*}\right), \quad\left\{\vartheta\left(v_{11}^{*}\right), \vartheta\left(v_{12}^{*}\right)\right\}=\left\{\phi\left(\vartheta\left(v_{21}^{*}\right)\right), \phi\left(\vartheta\left(v_{22}^{*}\right)\right)\right\}=\left\{\vartheta\left(v_{21}^{*}\right), \vartheta\left(v_{22}^{*}\right)\right\} ;
$$

the last equality holds because $v_{21}^{*}$ and $v_{22}^{*}$ are interchanged by $\sigma_{1}$. By (4.23), (4.22), (4.34), and (4.27),

$$
\operatorname{Cntr}_{\left(\Gamma, \sigma_{1}\right) ; e_{1}^{*}} \operatorname{Cntr}_{\left(\Gamma, \sigma_{1}\right) ; e_{2}^{*}}=-\frac{\operatorname{Cntr}_{\left(\Gamma, \sigma_{2}\right) ; e_{1}^{*}}}{\mathfrak{d}\left(e_{1}^{*}\right)}=-\frac{\operatorname{Cntr}_{\left(\Gamma, \sigma_{2}\right) ; e_{2}^{*}}}{\mathfrak{d}\left(e_{2}^{*}\right)}
$$

when restricted to the subtorus $\mathbb{T}^{m} \subset \mathbb{T}^{n}$.

Since $\left(\Gamma, \sigma_{1}\right)$ and $\left(\Gamma, \sigma_{2}\right)$ are admissible pairs, $\sigma_{1}$ and $\sigma_{2}$ act on Ver without fixed points. By Lemma 4.9, there thus exists a subset $\mathrm{V}_{+}^{\sigma_{1} \sigma_{2}}(\Gamma)$ of Ver that satisfies (4.24) for $\sigma=\sigma_{1}$ and $\sigma=\sigma_{2}$ at the same time. The involutions $\sigma_{1}$ and $\sigma_{2}$ restrict to involutions on (4.33) such that $\sigma_{2}$ acts without fixed points and the fixed points of $\sigma_{1}$ are $\left\{e_{1}^{*}, e_{2}^{*}\right\}$. By Lemma 4.9, there thus exists a subset $\mathrm{E}_{+}^{\sigma_{2}}(\Gamma)$ of $\mathrm{E}_{\mathbb{C}}^{\sigma_{2}}(\Gamma)$ satisfying (4.25) for $\sigma=\sigma_{2}$ such that

$$
\mathrm{E}_{+}^{\sigma_{1}}(\Gamma) \equiv \mathrm{E}_{+}^{\sigma_{2}}(\Gamma)-\left\{e_{1}^{*}, e_{2}^{*}\right\}
$$

satisfies (4.25) for $\sigma=\sigma_{1}$. Let $e_{i}^{*}$ be the unique element of $\mathrm{E}_{+}^{\sigma_{2}}(\Gamma) \cap\left\{e_{1}^{*}, e_{2}^{*}\right\}$. The vertex and edge contributions on the right-hand side of (4.26) are then the same for $\sigma=\sigma_{1}$ and $\sigma=\sigma_{2}$, except the contribution of $e_{i}^{*} \in \mathrm{E}_{+}^{\sigma_{2}}(\Gamma)$ is replaced by the product of the contributions of $e_{1}^{*}, e_{2}^{*} \in \mathrm{E}_{\mathbb{R}}^{\sigma_{1}}(\Gamma)$. The claim now follows from (4.35).

Proof of Theorem 1.6](2) for $k=0$. Let $(\Gamma, \sigma)$ be an element of $\mathcal{A}_{g, l}(n, d)$. Since $\mathrm{V}_{\mathbb{R}}^{\sigma}(\Gamma)=\varnothing$,

$$
g=g(\Gamma)=1+\left|\mathrm{E}_{\mathbb{R}}^{\sigma}(\Gamma)\right|+2\left|\mathrm{E}_{+}^{\sigma}(\Gamma)\right|+2 \sum_{v \in \mathrm{V}_{+}^{\sigma}(\Gamma)}(\mathfrak{g}(v)-1) \equiv 1+\left|\mathrm{E}_{\mathbb{R}}^{\sigma}(\Gamma)\right| \bmod 2 .
$$

Since $\mathfrak{d}(e) \notin 2 \mathbb{Z}$ for all $v \in \mathrm{E}_{\mathbb{R}}^{\sigma}(\Gamma)$,

$$
d=d(\Gamma)=\sum_{e \in \mathrm{E}_{\mathbb{R}}^{\sigma}(\Gamma)} \mathfrak{d}(e)+2 \sum_{e \in \mathrm{E}_{+}^{\sigma}(\Gamma)} \mathfrak{d}(e) \equiv\left|\mathrm{E}_{\mathbb{R}}^{\sigma}(\Gamma)\right| \bmod 2 .
$$

By (4.36) and (4.37), $\mathcal{A}_{g, l}(n, d)=\varnothing$ if $d-g \in 2 \mathbb{Z}$. Since all equivariant contributions to the genus $g$ degree $d$ real GW-invariants of $\left(\mathbb{P}^{2 m-1}, \omega_{2 m}, \tau_{2 m}\right)$ and $\left(\mathbb{P}^{4 m-1}, \omega_{4 m}, \eta_{4 m}\right)$ with only conjugate pairs of insertions arise from the elements of $\mathcal{A}_{g, l}(n, d)$, with $n=2 m$ and $n=4 m$, respectively, this establishes the claim. 
Proof of Proposition 1.7. By Theorem 4.6, the genus $g$ real GW-invariants of $\left(\mathbb{P}^{4 n-1}, \omega_{4 n}, \tau_{4 n}\right)$ and $\left(\mathbb{P}^{4 n-1}, \omega_{4 n}, \eta_{4 n}\right)$ with only conjugate pairs of insertions are obtained by summing the contributions from the same set of admissible pairs $(\Gamma, \sigma)$. The contributions (4.26) of $(\Gamma, \sigma)$ to the two invariants are products of the factors (4.18)-(4.23). The factors (4.23) corresponding to the $\sigma$-fixed edges of $\Gamma$ have opposite signs in the two cases; all other factors are the same. By (4.36), the parity of $\left|\mathrm{E}_{\mathbb{R}}^{\sigma}(\Gamma)\right|$ is the same as the parity of $g-1$. Thus, the contributions (4.26) to the two invariants from every admissible pair $(\Gamma, \sigma)$ differ by the factor of $(-1)^{g-1}$; this establishes the claim.

Example $4.10(d=2)$. We now apply Theorem 4.6 to compute the genus $g$ degree 2 real GWinvariants of $\left(\mathbb{P}^{3}, \tau_{4}\right)$ with 2 conjugate pairs of point constraints. They are given by

$$
\mathrm{GW}_{g, 2}^{\mathbb{P}^{3}, \tau_{4}}\left(H^{3}, H^{3}\right)=\int_{\left[\bar{M}_{g, 2}\left(\mathbb{P}^{3}, 2\right)^{\tau_{4}}\right]^{\mathrm{vrt}}} \mathrm{ev}_{1}^{*} \prod_{j \neq 1}\left(\mathbf{x}-\alpha_{j}\right) \mathrm{ev}_{2}^{*} \prod_{j \neq 3}\left(\mathrm{x}-\alpha_{j}\right),
$$

where $H \in H^{2}\left(\mathbb{P}^{3} ; \mathbb{Q}\right)$ is the usual hyperplane class and $\mathbf{x} \in H_{\mathbb{T}^{4}}^{2}\left(\mathbb{P}^{3} ; \mathbb{Q}\right)$ is the equivariant hyperplane class. If $(\Gamma, \sigma) \in \mathcal{A}_{g, 2}(4,2)$ and $\Gamma$ is as in (4.12), then

$$
\left.\mathrm{ev}_{i}^{*} \prod_{j \neq 2 i-1}\left(\mathbf{x}-\alpha_{j}\right)\right|_{\mathcal{Z}_{\Gamma, \sigma}}=\prod_{j \neq 2 i-1}\left(\alpha_{\vartheta\left(\mathfrak{m}\left(i^{+}\right)\right)}-\alpha_{j}\right) \quad \forall i=1,2,
$$

where $\mathcal{Z}_{\Gamma, \sigma}$ is the $\mathbb{T}^{2}$-fixed locus corresponding to $(\Gamma, \sigma)$; this restriction is formally encoded into the vertex contribution, (4.18) or (4.19), of $v=\mathfrak{m}\left(i^{+}\right)$. Thus, the restriction of the integrand in (4.38) to $\mathcal{Z}_{\Gamma, \sigma}$ vanishes unless

$$
\vartheta\left(\mathfrak{m}\left(1^{+}\right)\right)=1, \quad \vartheta\left(\mathfrak{m}\left(2^{+}\right)\right)=3, \quad \vartheta\left(\mathfrak{m}\left(1^{-}\right)\right)=2, \quad \vartheta\left(\mathfrak{m}\left(2^{-}\right)\right)=4
$$

Since there are no degree 2 connected graphs with at least 4 vertices, the restriction of the integrand in (4.38) to all $\mathbb{T}^{2}$-fixed loci vanishes and so

$$
\mathrm{GW}_{g, 2}^{\mathbb{P}^{3}, \tau_{4}}\left(H^{3}, H^{3}\right)=0 \quad \forall g \in \mathbb{Z} .
$$

Example $4.11(g=1, d=4)$. We next compute the genus 1 degree 4 real GW-invariant of $\left(\mathbb{P}^{3}, \tau_{4}\right)$ with 4 conjugate pairs of point constraints as

$$
\mathrm{GW}_{1,4}^{\mathbb{P}^{3}, \tau_{4}}\left(H^{3}, H^{3}, H^{3}, H^{3}\right)=\int_{\left[\overline{\mathfrak{M}}_{1,4}\left(\mathbb{P}^{3}, 4\right)^{\tau_{4}}\right]^{\mathrm{vrt}}} \prod_{i=1}^{4}\left(\mathrm{ev}_{i}^{*} \prod_{j \neq i}\left(\mathbf{x}-\alpha_{j}\right)\right) .
$$

Similarly to Example 4.10, the restriction of the integrand in (4.40) to a fixed locus $\mathcal{Z}_{\Gamma, \sigma}$ vanishes unless $\vartheta\left(\mathfrak{m}\left(i^{+}\right)\right)=i$ for all $i \in[4]$. There are 11 pairs in $\mathcal{A}_{1,4}(4,4)$ satisfying this condition: the first diagram in Figure 2, with the four possible ways of labeling its vertices and the two possible involutions on the loop, and the three other diagrams. For each of these diagrams,

$$
\left.\mathrm{ev}_{i}^{*} \prod_{j \neq i}\left(\mathbf{x}-\alpha_{j}\right)\right|_{\mathcal{Z}_{\Gamma, \sigma}}=\prod_{j \neq i}\left(\alpha_{\vartheta\left(\mathfrak{m}\left(i^{+}\right)\right)}-\alpha_{j}\right)=\mathbf{e}\left(T_{P_{i}} \mathbb{P}^{3}\right)
$$

see (4.7). All eight versions of the first diagram in Figure 2 have the same automorphism group, i.e. $\mathbb{Z}_{2}$. Since the degrees of the vertical edges are 1, Corollary 4.8 thus implies that these graphs cancel in pairs. For the remaining three graphs, we can choose the same distinguished subset $\mathrm{V}_{+}^{\sigma}(\Gamma)$ 

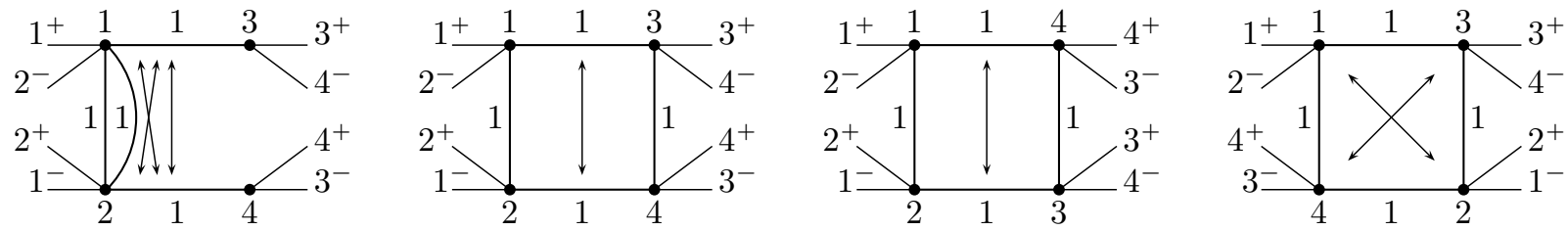

Figure 2: The elements of $\mathcal{A}_{1,4}(4,4)$ potentially contributing to (4.40).

of Ver consisting of the top vertices. Their contributions in the three cases are given by (4.18) with $\alpha_{\vartheta(v)}^{|\mathbf{p}|_{v}}$ replaced by $\mathbf{e}\left(T_{P_{\vartheta(v)}} \mathbb{P}^{3}\right)^{2}$ and $\overline{\mathcal{M}}_{\mathfrak{g}(v), S_{v}}=\overline{\mathcal{M}}_{0,4}$ :

$$
\begin{array}{lll}
\mathbf{e}\left(T_{P_{1}} \mathbb{P}^{3}\right)^{3} \frac{3 \lambda_{1}-\lambda_{2}}{8 \lambda_{1}^{3}\left(\lambda_{1}-\lambda_{2}\right)^{3}}, & \mathbf{e}\left(T_{P_{1}} \mathbb{P}^{3}\right)^{3} \frac{3 \lambda_{1}+\lambda_{2}}{8 \lambda_{1}^{3}\left(\lambda_{1}+\lambda_{2}\right)^{3}}, & \mathbf{e}\left(T_{P_{1}} \mathbb{P}^{3}\right)^{3} \frac{2 \lambda_{1}}{\left(\lambda_{1}^{2}-\lambda_{2}^{2}\right)^{3}}, \\
\mathbf{e}\left(T_{P_{3}} \mathbb{P}^{3}\right)^{3} \frac{3 \lambda_{2}-\lambda_{1}}{8 \lambda_{2}^{3}\left(\lambda_{2}-\lambda_{1}\right)^{3}}, & -\mathbf{e}\left(T_{P_{4}} \mathbb{P}^{3}\right)^{3} \frac{3 \lambda_{2}+\lambda_{1}}{8 \lambda_{2}^{3}\left(\lambda_{2}+\lambda_{1}\right)^{3}}, & \mathbf{e}\left(T_{P_{3}} \mathbb{P}^{3}\right)^{3} \frac{2 \lambda_{2}}{\left(\lambda_{2}^{2}-\lambda_{1}^{2}\right)^{3}} ;
\end{array}
$$

see also (4.10). In the case of the two middle diagrams, the set $\mathrm{E}_{\mathbb{R}}^{\sigma}(\Gamma)$ consists of the two vertical edges. The product of their contributions, as given by (4.23), is $-\left(\lambda_{1}^{2}-\lambda_{2}^{2}\right)^{-2}$ in both cases. For the set $\mathrm{E}_{+}^{\sigma}(\Gamma)$, we can choose the top edge in these cases. Its contributions, as given by (4.22), are

$$
-\frac{1}{4 \lambda_{1} \lambda_{2}\left(\lambda_{1}+\lambda_{2}\right)^{2}} \quad \text { and } \quad \frac{1}{4 \lambda_{1} \lambda_{2}\left(\lambda_{1}-\lambda_{2}\right)^{2}},
$$

respectively. In the case of the last diagram, we can take $\mathrm{E}_{+}^{\sigma}(\Gamma)$ to consist of the top and left edges; their contributions are given by (4.41). In all three cases, the vertex signs $(-1)^{\mathfrak{s}_{v}}$ are the same for the two vertices. Putting the three contributions together, we obtain

$$
\begin{gathered}
\mathrm{GW}_{1,4}^{\mathbb{P}^{3}, \tau_{4}}\left(H^{3}, H^{3}, H^{3}, H^{3}\right)=\frac{\left(\lambda_{1}+\lambda_{2}\right)^{2}\left(3 \lambda_{1}-\lambda_{2}\right)\left(3 \lambda_{2}-\lambda_{1}\right)}{4 \lambda_{1} \lambda_{2}\left(\lambda_{1}-\lambda_{2}\right)^{2}}+\frac{\left(\lambda_{1}-\lambda_{2}\right)^{2}\left(3 \lambda_{1}+\lambda_{2}\right)\left(3 \lambda_{2}+\lambda_{1}\right)}{4 \lambda_{1} \lambda_{2}\left(\lambda_{1}+\lambda_{2}\right)^{2}} \\
-\frac{16 \lambda_{1}^{2} \lambda_{2}^{2}}{\left(\lambda_{1}^{2}-\lambda_{2}^{2}\right)^{2}}=-1 .
\end{gathered}
$$

\section{Proof of Theorem 4.6}

It remains to establish Theorem 4.6. For the remainder of this paper, we assume

$$
n \in \mathbb{Z}^{+}, \quad g, d, k, l \in \mathbb{Z}^{\geqslant 0}, \quad n-k \in 2 \mathbb{Z}, \quad \mathbf{a} \in\left(\mathbb{Z}^{+}\right)^{k},
$$

and $\phi=\tau_{n}^{\prime}$ or $n \in 2 \mathbb{Z}$ and $\phi=\eta_{n}$. We also assume that $(n, \mathbf{a})$ satisfies the assumptions of Proposition 1.4(1) if $\phi=\tau_{n}^{\prime}$ and of Proposition 1.4(2) with $(m=n / 2$, a $)$ if $\phi=\eta_{n}$. Let $m=\lfloor n / 2\rfloor$ as before and $S_{l}$ be as in (4.16). 


\subsection{The torus-fixed loci}

We first identify the topological components of the fixed locus of the $\mathbb{T}^{m}$-action on $\overline{\mathfrak{M}}_{g, l}\left(\mathbb{P}^{n-1}, d\right)^{\phi}$.

Similarly to the situation in [18, Section 27.3], an element

$$
[\mathbf{u}] \equiv\left[\Sigma,\left(z_{1}^{+}, z_{1}^{-}\right), \ldots,\left(z_{l}^{+}, z_{l}^{-}\right), \sigma, u\right] \in \overline{\mathfrak{M}}_{g, l}\left(\mathbb{P}^{n-1}, d\right)^{\phi}
$$

is fixed by the $\mathbb{T}^{m}$-action if and only if

(F1) the image of every irreducible component of $\Sigma$ is either a $\mathbb{T}^{m}$-fixed point or a $\mathbb{T}^{m}$-invariant irreducible curve in $\mathbb{P}^{n-1}$,

(F2) the image of every nodal and marked point of $\Sigma$ is a $\mathbb{T}^{m}$-fixed point in $\mathbb{P}^{n-1}$,

(F3) the image of every branch point of the restriction $u$ to an irreducible component of $\Sigma$ is a $\mathbb{T}^{m}$-fixed point in $\mathbb{P}^{n-1}$.

The $\mathbb{T}^{m}$-fixed points and $\mathbb{T}^{m}$-invariant irreducible curves in $\mathbb{P}^{n-1}$ are described by Lemma 4.1, For a $\mathbb{T}^{m}$-invariant stable map as in (5.1), every non-constant restriction of the map $u$ to an irreducible component of $\Sigma$ is thus a cover of a line $\mathbb{P}_{i j}^{1}$ with $i \neq j$ branched only over the points $P_{i}$ and $P_{j}$ or of a conic $\mathcal{C}_{i}(a, b)$ with $a, b \in \mathbb{C}^{*}$ branched only over the points $P_{2 i-1}$ and $P_{2 i}$; the latter is a possibility only if $n=2 m+1$. Since $\mathbb{P}_{i j}^{1}$ and $\mathcal{C}_{i}(a, b)$ are smooth rational curves, the Riemann-Hurwitz formula [16, p219] implies that the domain of any irreducible cover of either $\mathbb{P}_{i j}^{1}$ or $\mathcal{C}_{i}(a, b)$ branched only over two points is also a $\mathbb{P}^{1}$.

The combinatorial structure of a $\mathbb{T}^{m}$-invariant stable map as in (5.1) can thus be described by a connected decorated graph $\Gamma$ as in (4.12). The irreducible components $\Sigma_{e}$ of $\Sigma$ on which the map $u$ is not constant are rational and correspond to the edges $e \in \mathrm{Edg}$. For $e=\left\{v_{1}, v_{2}\right\},\left.u\right|_{\Sigma_{e}}$ is either a degree $\mathfrak{d}(e)$ cover of the line $\mathbb{P}_{\vartheta\left(v_{1}\right), \vartheta\left(v_{2}\right)}^{1}$ or a degree $\mathfrak{d}(e) / 2$ cover of a conic $\mathcal{C}_{\left\lfloor\left(\vartheta\left(v_{1}\right)+1\right) / 2\right\rfloor}(a, b)$; the latter is a possibility only if (4.20) holds. In both cases, the map $\left.u\right|_{\Sigma_{e}}$ is ramified only over $P_{\vartheta\left(v_{1}\right)}$ and $P_{\vartheta\left(v_{2}\right)}$. We denote the moduli space of all possible $\left.u\right|_{\Sigma_{e}}$ and its closure by

$$
\begin{array}{ll}
\mathfrak{M}_{\Gamma, \sigma ; e}^{\phi ; \mathbb{T} ; \circ} \subset \overline{\mathfrak{M}}_{\Gamma, \sigma ; e}^{\phi ; \mathbb{T} ; \circ} \subset \overline{\mathfrak{M}}_{0,0}\left(\mathbb{P}^{n-1}, \mathfrak{d}(e)\right)^{\phi} & \text { if } e \in \mathrm{E}_{\mathbb{R}}^{\sigma}(\Gamma), \\
\mathfrak{M}_{\Gamma ; e}^{\mathbb{T} ; \circ} \subset \overline{\mathfrak{M}}_{\Gamma ; e}^{\mathbb{T} ; \circ} \subset \overline{\mathfrak{M}}_{0,0}\left(\mathbb{P}^{n-1}, \mathfrak{d}(e)\right) & \text { if } e \in \mathrm{E}_{\mathbb{C}}^{\sigma}(\Gamma) .
\end{array}
$$

We denote by

$$
\mathcal{N}_{\Gamma, \sigma ; e}^{\phi ; \circ} \longrightarrow \overline{\mathfrak{M}}_{\Gamma, \sigma ; e}^{\phi ; \mathbb{T} ; \circ} \quad \text { and } \quad \mathcal{N}_{\Gamma ; e}^{\circ} \longrightarrow \overline{\mathfrak{M}}_{\Gamma ; e}^{\mathbb{T} ; \circ}
$$

the corresponding normal bundles.

The vertices $v \in$ Ver with $\operatorname{val}_{v}(\Gamma) \geqslant 3$ correspond to the maximal connected unions $\Sigma_{v}$ of irreducible components of $\Sigma$ on which $u$ is constant. The arithmetic genus of such $\Sigma_{v}$ is $\mathfrak{g}(v)$; it is sent by $u$ to $P_{\vartheta(v)}$ and carries the marked points $\mathfrak{m}^{-1}(v) \subset S_{l}$. If $v \in \mathrm{V}_{\mathbb{C}}^{\sigma}(\Gamma)$, we denote the moduli space of all possible $\Sigma_{v}$ by

$$
\overline{\mathfrak{M}}_{\Gamma ; v}^{\mathbb{T}} \subset \overline{\mathfrak{M}}_{\mathfrak{g}(v), S_{v}}\left(\mathbb{P}^{n-1}, 0\right)
$$


with $S_{v}$ as in (4.17); it is isomorphic to $\overline{\mathcal{M}}_{\mathfrak{g}(v), S_{v}}$. The remaining marked points of $\Sigma$ are the branch points of $\left.u\right|_{\Sigma_{e}}$ corresponding to the vertices $v \in e$ with $\operatorname{val}_{v}(\Gamma)=2$ and $\left|\mathrm{E}_{v}(\Gamma)\right|=1$. The remaining vertices of $v$ with $\operatorname{val}_{v}(\Gamma)=2$ correspond to the nodes of $\Sigma$ shared by two irreducible components $\Sigma_{e_{1}}$ and $\Sigma_{e_{2}}$ with $e_{1}, e_{2} \in$ Edg. The involution $\sigma$ on $\Sigma$ induces an involution $\sigma$ on the graph $\Gamma$. We will call the pair $(\Gamma, \sigma)$ obtained in this way the combinatorial type of the $\mathbb{T}^{m}$-fixed stable map (5.1).

Remark 5.1. If $e$ does not satisfy (4.20), then the spaces in (5.2) consist of a single element with the automorphism group $\mathbb{Z}_{\mathfrak{o}(e)}$. Let $i=\left\lfloor\left(\vartheta\left(v_{1}\right)+1\right) / 2\right\rfloor$. If $e \in \mathrm{E}_{\mathbb{C}}^{\sigma}(\Gamma)$ satisfies (4.20), then

$$
\overline{\mathfrak{M}}_{\Gamma ; e}^{\mathbb{T} ; \circ} \approx\left\{[a, b] \in \mathbb{P}^{1}\right\}
$$

as topological spaces; see Lemma 4.1](2) This identification can be chosen so that the image of the map corresponding to $[a, b]$ is the conic $\mathcal{C}_{i}(a, b)$. The points $[1,0]$ and $[0,1]$ then correspond to the covers of $\mathbb{P}_{01}^{1} \cup \mathbb{P}_{02}^{1}$ and $\mathbb{P}_{12}^{1}$, respectively. The automorphism groups of these points are $\left(\mathbb{Z}_{\mathfrak{d}(e) / 2}\right)^{2}$ and $\mathbb{Z}_{\mathfrak{d}(e)}$, respectively; the automorphism groups of the remaining elements are $\mathbb{Z}_{\mathfrak{d}(e) / 2}$. By Lemma 4.1](3), the space of real $\mathbb{T}^{1}$-invariant conics (not necessarily smooth) in $\left(\mathbb{P}^{2}, \tau_{3}\right)$ is

$$
\left\{[a, b] \in \mathbb{P}^{1}: a \bar{b} \in \mathbb{R}\right\} \approx S^{1} \subset \mathbb{P}^{1} .
$$

Suppose $e \in \mathrm{E}_{\mathbb{R}}^{\sigma}(\Gamma)$ satisfies (4.20). The $\mathbb{T}^{1}$-invariant degree $\mathfrak{d}(e)$ cover of $\mathbb{P}_{12}^{1}$ is then compatible with both involutions on the domain; the automorphism groups of both resulting real covers are $\mathbb{Z}_{\mathfrak{d}(e)}$. The $\mathbb{T}^{1}$-invariant degree $\mathfrak{d}(e) / 2$ cover of $\mathbb{P}_{01}^{1} \cup \mathbb{P}_{02}^{1}$ is compatible with one involution on the domain; the automorphism group of the resulting real cover is $\mathbb{Z}_{\mathfrak{o}(e) / 2}$. If $\mathfrak{d}(e) \notin 4 \mathbb{Z}$, the same is the case for the $\mathbb{T}^{1}$-invariant degree $\mathfrak{d}(e) / 2$ cover of each of the smooth real conics $\mathcal{C}_{i}(a, b)$. If $\mathfrak{d}(e) \in 4 \mathbb{Z}$, the degree $\mathfrak{d}(e) / 2$ covers of the conics $\mathcal{C}_{i}(a, b)$ with $a \bar{b} \in \mathbb{R}^{-}$are not compatible with any involution on the domain as these conics have no fixed locus; see Remark 4.2 and [3, Lemma 1.9]. The $\mathbb{T}^{1}$-invariant degree $\mathfrak{d}(e) / 2$ cover of each conic $\mathcal{C}_{i}(a, b)$ with $a \bar{b} \in \mathbb{R}^{+}$is compatible with both involutions on the domain; the automorphism groups of both resulting real covers are $\mathbb{Z}_{\mathfrak{d}(e) / 2}$. In both cases, $\overline{\mathfrak{M}}_{\Gamma, \sigma ; e}^{\phi ; T ; \circ}$ can be viewed as the interval $[-1,1]$ with the trivial $\mathbb{Z}_{\mathfrak{d}(e) / 2}$-action on the inte-

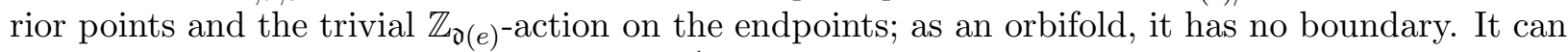

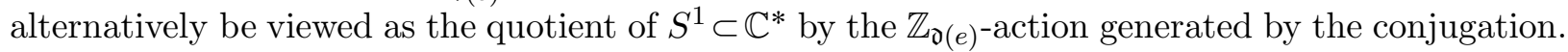

Let $\Gamma$ be an $S_{l}$-marked $[n]$-decorated connected graph with an involution $\sigma$ such that $g(\Gamma)=g$ and $d(\Gamma)=d$. We denote by

$$
\mathcal{Z}_{\Gamma, \sigma} \subset \overline{\mathfrak{M}}_{g, l}\left(\mathbb{P}^{n-1}, d\right)^{\phi}
$$

the subspace consisting of all $\mathbb{T}^{m}$-fixed elements of the combinatorial type of $(\Gamma, \sigma)$. This subspace is closed unless some edge $e \in \operatorname{Edg}$ satisfies (4.20). In such a case, the closure $\overline{\mathcal{Z}}_{\Gamma, \sigma}$ of $\mathcal{Z}_{\Gamma, \sigma}$ also includes the subspaces $\mathcal{Z}_{\Gamma^{\prime}, \sigma^{\prime}}$ corresponding to the pairs $\left(\Gamma^{\prime}, \sigma^{\prime}\right)$ obtained from $(\Gamma, \sigma)$ by the "local replacement" of [32, Figure 5]:

- adding a new vertex $v_{e}$ to an edge $e$ satisfying (4.20),

- extending the functions $\mathfrak{g}$ and $\vartheta$ to $v_{e}$ by 0 and $n$, respectively, and

- replacing the value of $\mathfrak{d}$ on $e$ by the values of $\mathfrak{d}(e) / 2$ on each of the two new edges; 

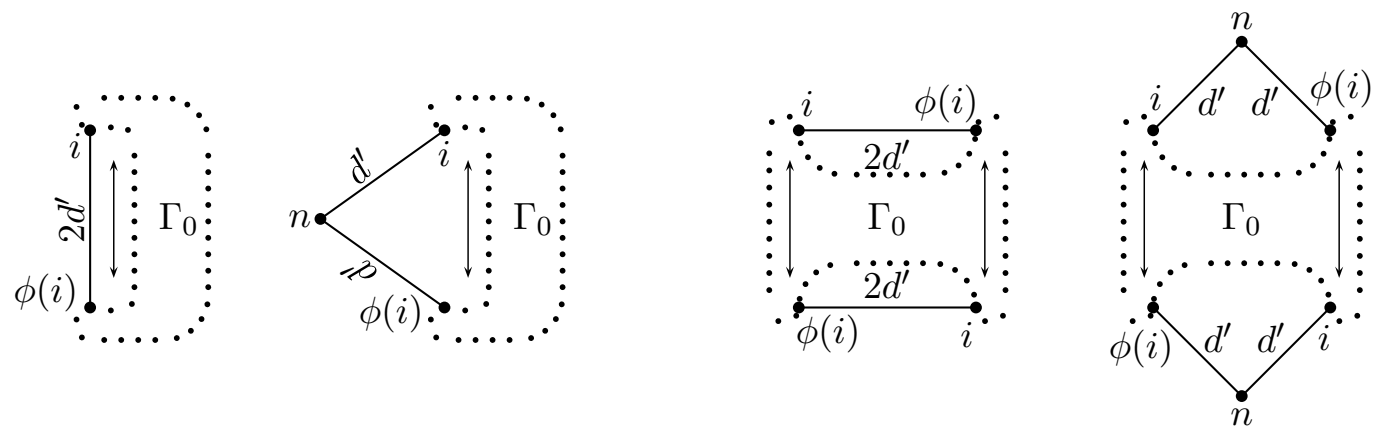

Figure 3: The second and fourth graphs are degenerations of the first and third, respectively, induced by the degenerations of $\mathbb{T}^{m}$-fixed conics in $\mathbb{P}^{n-1}$ with $n$ odd; $\Gamma_{0}$ indicates any graph compatible with the indicated involution.

see Figure 3. If $e \in \mathrm{E}_{\mathbb{R}}^{\sigma}(\Gamma)$, the involution $\sigma^{\prime}$ is obtained from $\sigma$ by sending $v_{e}$ to itself and interchanging the two new edges. If $e \in \mathrm{E}_{\mathbb{C}}^{\sigma}(\Gamma)$, the above breaking procedure should simultaneously be performed on the edge $\sigma(e)$. The involution $\sigma^{\prime}$ on $\Gamma^{\prime}$ is then obtained from $\sigma$ by interchanging $v_{e}$ with $v_{\sigma(e)}$ and the two pairs of new edges according to the action on their vertices. This graph degeneration corresponds to the degeneration of the conics $\mathcal{C}_{i}(a, b)$ to the union of the lines $\mathbb{P}_{2 i-1, n}^{1}$ and $\mathbb{P}_{2 i, n}^{1}$. Denote by

$$
\mathcal{N}_{\Gamma, \sigma}^{\phi} \longrightarrow \overline{\mathcal{Z}}_{\Gamma, \sigma}
$$

the normal bundle of $\overline{\mathcal{Z}}_{\Gamma, \sigma}$ in $\overline{\mathfrak{M}}_{g, l}\left(\mathbb{P}^{n-1}, d\right)^{\phi}$.

By the above,

$$
\overline{\mathfrak{M}}_{g, l}\left(\mathbb{P}^{n-1}, d\right)^{\phi ; \mathbb{T}^{m}}=\bigsqcup_{(\Gamma, \sigma)} \overline{\mathcal{Z}}_{\Gamma, \sigma},
$$

with the union taken over all pairs $(\Gamma, \sigma)$ consisting of an $S_{l}$-marked $[n]$-decorated connected graph $\Gamma$ with an involution $\sigma$ such that $g(\Gamma)=g, d(\Gamma)=d$, and $\Gamma$ contains no vertex $v$ such that

$$
\vartheta(v)=2 m+1, \quad \operatorname{val}_{v}(\Gamma)=\mathrm{E}_{v}(\Gamma)=2, \quad \mathfrak{d}\left(e_{1}\right)=\mathfrak{d}\left(e_{2}\right), \quad \vartheta\left(e_{1} / v\right)=\phi\left(\vartheta\left(e_{2} / v\right)\right),
$$

where $e_{1}, e_{2} \in \mathrm{E}_{v}(\Gamma)$ are the two elements of $\mathrm{E}_{v}(\Gamma)$. We denote the set of all such pairs $(\Gamma, \sigma)$ by $\overline{\mathcal{A}}_{g, l}(n, d)$.

The spaces $\overline{\mathcal{Z}}_{\Gamma, \sigma}$ need not be connected or non-empty. If $n \in 2 \mathbb{Z}, \phi=\tau_{n}^{\prime}$, and $\mathfrak{d}(e) \in 2 \mathbb{Z}$ for some $e \in \mathrm{E}_{\mathbb{R}}^{\sigma}(\Gamma)$, then $(\Gamma, \sigma)$ is compatible with two distinct topological types of the real degree $\mathfrak{d}(e)$ covers $\Sigma_{e} \longrightarrow \mathbb{P}_{\vartheta\left(v_{1}\right) \vartheta\left(v_{2}\right)}^{1}$ branched only over two points. By the proof of Lemma 5.3 , the $\mathbb{T}^{m}$-fixed loci associated with the two types of covers contribute to (4.4) with opposite signs. If $k \in \mathbb{Z}^{+}$and $\mathfrak{d}(e) \in 2 \mathbb{Z}$, then the restriction of $\mathcal{V}_{n ; \mathbf{a}}^{\widetilde{\phi}_{n ; \mathbf{a}}}$ to $\overline{\mathcal{Z}}_{\Gamma, \sigma}$ contains a subbundle of odd rank. This is in particular the case if $n \notin 2 \mathbb{Z}$ and thus $\phi=\tau_{n}^{\prime}$. Since the Euler class of such a subbundle vanishes, the contribution of $\overline{\mathcal{Z}}_{\Gamma, \sigma}$ to (4.4) is zero in this case as well. If $n \in 2 \mathbb{Z}, \phi=\eta_{n}$, and $\mathfrak{d}(e) \in 2 \mathbb{Z}$, then $(\Gamma, \sigma)$ is not compatible with any real degree $\mathfrak{d}(e)$ cover $\Sigma_{e} \longrightarrow \mathbb{P}_{\vartheta\left(v_{1}\right) \vartheta\left(v_{2}\right)}^{1}$; see [3, Lemma 1.9]. Thus, $\mathcal{Z}_{\Gamma, \sigma}$ is empty and does not contribute to (4.4) in this case either if $\mathfrak{d}(e) \in 2 \mathbb{Z}$ for some $e \in \mathrm{E}_{\mathbb{R}}^{\sigma}(\Gamma)$. This motivates the first restriction in (4.15). 
Suppose $\Gamma$ is a decorated graph as in (4.12), $\sigma$ is an involution on $\Gamma$, and $[\mathbf{u}]$ is an element of $\overline{\mathcal{Z}}_{\Gamma, \sigma}$ as in (5.1). For each $e \in \mathrm{E}_{v}(\Gamma)$, let $x_{e, v} \in \Sigma_{e}$ be the branch point of $\left.u\right|_{\Sigma_{e}}$ sent to $P_{\vartheta(v)}$. Given $v \in$ Ver, let

$$
\begin{aligned}
\mathrm{F}_{v}(\Gamma)= & \left\{(e, v): e \in \mathrm{E}_{v}(\Gamma)\right\} \cup\left\{(\sigma(e), \sigma(v)): e \in \mathrm{E}_{v}(\Gamma)\right\} \subset \mathrm{Edg} \times \text { Ver } \\
\mathrm{V}_{v}^{\prime}(\Gamma)= & \left\{e / v: e \in \mathrm{E}_{v}(\Gamma)\right\} \cup\left\{\sigma(e / v): e \in \mathrm{E}_{v}(\Gamma)\right\} \\
& -\{v, \sigma(v)\}-\left\{e / v: e \in \mathrm{E}_{v}(\Gamma)\right\} \cap\left\{\sigma(e / v): e \in \mathrm{E}_{v}(\Gamma)\right\} \\
\mathrm{F}_{v}^{\prime}(\Gamma)= & \left\{\left(e^{\prime}, v^{\prime}\right): e^{\prime} \in \mathrm{E}_{v}(\Gamma) \cup \mathrm{E}_{\sigma(v)}(\Gamma), v^{\prime} \in e^{\prime} \cap \mathrm{V}_{v}^{\prime}(\Gamma)\right\}
\end{aligned}
$$

We define $\Sigma_{v}^{\prime} \subset \Sigma$ by

$$
\Sigma_{v}^{\prime}=\bigcup_{e \in \mathrm{E}_{v}(\Gamma)}\left(\Sigma_{e} \cup \Sigma_{\sigma(e)}\right) \cup \begin{cases}\Sigma_{v} \cup \Sigma_{\sigma(v)}, & \text { if } \operatorname{val}_{v}(\Gamma) \geqslant 3 ; \\ \varnothing, & \text { if } \operatorname{val}_{v}(\Gamma) \leqslant 2 .\end{cases}
$$

Thus, $\Sigma_{v}^{\prime}$ is a union of irreducible components of $\Sigma_{v}$; the nodes shared by $\Sigma_{v}^{\prime}$ with other irreducible components of $\Sigma$ are contained in the set $\left\{x_{e^{\prime}, v^{\prime}}\right\}_{\left(e^{\prime}, v^{\prime}\right) \in \mathrm{F}_{v}^{\prime}(\Gamma)}$. Let $\widetilde{\Sigma}_{v}^{\prime}$ be the nodal surface obtained from $\Sigma_{v}^{\prime}$ by removing the components in $\Sigma_{v} \cup \Sigma_{\sigma(v)}$ if $\operatorname{val}_{v}(\Gamma) \geqslant 3$ and separating $\Sigma_{v}^{\prime}$ at the node or nodes corresponding to $v$ and $\sigma(v)$ if $\operatorname{val}_{v}(\Gamma),\left|\mathrm{E}_{v}(\Gamma)\right|=2$. Thus, every topological component of $\tilde{\Sigma}_{v}^{\prime}$ is either $\mathbb{P}^{1}$ or a wedge of two copies of $\mathbb{P}^{1}$. The involution $\sigma$ on $\Sigma$ restricts to an involution on $\Sigma_{v}^{\prime}$ and induces an involution on $\widetilde{\Sigma}_{v}^{\prime}$.

Let $\left(\mathcal{L}_{n ; \mathbf{a}}, \tilde{\phi}_{n ; \mathbf{a}}\right)$ be as in (2.27). Thus,

$$
\left.\mathcal{V}_{n ; \mathbf{a}}^{\tilde{\phi}_{n ; \mathbf{a}}}\right|_{[\mathbf{u}]}=H^{0}\left(\Sigma ; u^{*} \mathcal{L}_{n ; \mathbf{a}}\right)^{\tilde{\phi}_{n ; \mathbf{a}}} / \operatorname{Aut}(\mathbf{u}) .
$$

For each $v \in$ Ver, define

$$
\begin{aligned}
& H^{0}\left(\Sigma_{v}^{\prime} ; u^{*} \mathcal{L}_{n ; \mathbf{a}}\right)_{v}^{\widetilde{\phi}_{n ; \mathbf{a}}} \equiv\left\{\xi \in H^{0}\left(\Sigma_{v}^{\prime} ; u^{*} \mathcal{L}_{n ; \mathbf{a}}\right)^{\widetilde{\phi}_{n ; \mathbf{a}}}: \xi\left(x_{e^{\prime}, v^{\prime}}\right)=0 \forall\left(e^{\prime}, v^{\prime}\right) \in \mathrm{F}_{v}^{\prime}(\Gamma)\right\},
\end{aligned}
$$

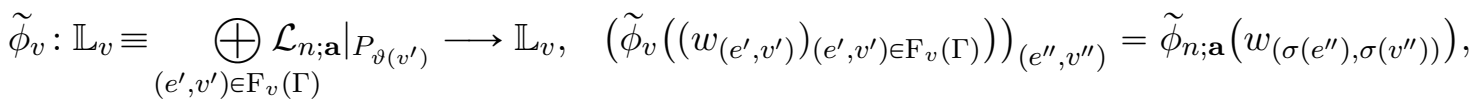

$$
\begin{aligned}
& \widetilde{\phi}_{v}^{\prime}:\left.\mathbb{L}_{v}^{\prime} \equiv \underset{\left(e^{\prime}, v^{\prime}\right) \in \mathrm{F}_{v}^{\prime}(\Gamma)}{\bigoplus} \mathcal{L}_{n ; \mathbf{a}}\right|_{P_{\vartheta\left(v^{\prime}\right)}} \longrightarrow \mathbb{L}_{v}^{\prime}, \quad\left(\widetilde{\phi}_{v}^{\prime}\left(\left(w_{\left(e^{\prime}, v^{\prime}\right)}\right)_{\left(e^{\prime}, v^{\prime}\right) \in \mathrm{F}_{v}^{\prime}(\Gamma)}\right)\right)_{\left(e^{\prime \prime}, v^{\prime \prime}\right)}=\widetilde{\phi}_{n ; \mathbf{a}}\left(w_{\left(\sigma\left(e^{\prime \prime}\right), \sigma\left(v^{\prime \prime}\right)\right)}\right) .
\end{aligned}
$$

The two involutions above interchange the components indexed by $\left(e^{\prime}, v^{\prime}\right)$ and $\left(\sigma\left(e^{\prime}\right), \sigma\left(v^{\prime}\right)\right)$. Furthermore,

$$
\begin{aligned}
H^{0}\left(\Sigma_{v}^{\prime} ; u^{*} \mathcal{L}_{n ; \mathbf{a}}\right)_{v}^{\tilde{\phi}_{n ; \mathbf{a}}}=\left\{\xi \in H^{0}\left(\widetilde{\Sigma}_{v}^{\prime} ; u^{*} \mathcal{L}_{n ; \mathbf{a}}\right)^{\tilde{\phi}_{n ; \mathbf{a}}}: \xi\left(x_{e^{\prime}, v^{\prime}}\right)\right. & =0 \forall\left(e^{\prime}, v^{\prime}\right) \in \mathrm{F}_{v}^{\prime}(\Gamma), \\
\xi\left(x_{e_{1}, v}\right) & \left.=\xi\left(x_{e_{2}, v}\right) \forall e_{1}, e_{2} \in \mathrm{E}_{v}(\Gamma)\right\} .
\end{aligned}
$$

Since the nodes shared by $\Sigma_{v}^{\prime}$ with the remainder of $\Sigma$ are contained in the set $\left\{x_{e^{\prime}, v^{\prime}}\right\}_{\left(e^{\prime}, v^{\prime}\right) \in F_{v}^{\prime}(\Gamma)}$, the image of the restriction homomorphism

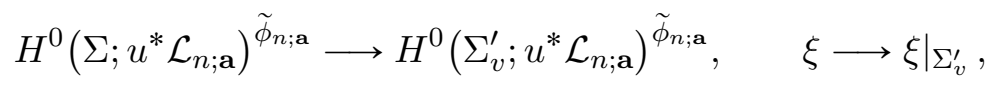

contains the subspace $H^{0}\left(\Sigma_{v}^{\prime} ; u^{*} \mathcal{L}_{n ; \mathbf{a}}\right)_{v}^{\widetilde{\phi}_{n} ; \mathbf{a}}$. Since each topological component of $\widetilde{\Sigma}_{v}^{\prime}$ is rational and contains at most two of the points $x_{e^{\prime}, v^{\prime}}$ with $\left(e^{\prime}, v^{\prime}\right)$ in $\mathrm{F}_{v}(\Gamma) \cup \mathrm{F}_{v}^{\prime}(\Gamma)$, the homomorphism

$$
H^{0}\left(\widetilde{\Sigma}_{v}^{\prime} ; u^{*} \mathcal{L}_{n ; \mathbf{a}}\right)^{\tilde{\phi}_{n ; \mathbf{a}}} \longrightarrow \mathbb{L}_{v}^{\tilde{\phi}_{v}} \oplus \mathbb{L}_{v}^{\prime \widetilde{\phi}_{v}^{\prime}}, \quad \xi \longrightarrow\left(\left(\xi\left(x_{e^{\prime}, v^{\prime}}\right)\right)_{\left(e^{\prime}, v^{\prime}\right) \in \mathrm{F}_{v}(\Gamma)},\left(\xi\left(x_{e^{\prime}, v^{\prime}}\right)\right)_{\left(e^{\prime}, v^{\prime}\right) \in \mathrm{F}_{v}^{\prime}(\Gamma)}\right),
$$


is surjective.

If $v \in \operatorname{Ver}$ and $\operatorname{val}_{v}(\Gamma) \geqslant 3,\left.\xi\right|_{\Sigma_{v}}$ is constant for all $\xi \in H^{0}\left(\Sigma ; u^{*} \mathcal{L}_{n ; \mathbf{a}}\right)$. Thus, the evaluation homomorphism

$$
\tilde{\mathrm{ev}}_{v}:\left.H^{0}\left(\Sigma ; u^{*} \mathcal{L}_{n ; \mathbf{a}}\right) \longrightarrow \mathcal{L}_{n ; \mathbf{a}}\right|_{P_{\vartheta(v)}}, \quad \tilde{\mathrm{ev}}_{v}(\xi)=\xi\left(\Sigma_{v}\right)
$$

is well defined. If $\operatorname{val}_{v}(\Gamma),\left|\mathrm{E}_{v}(\Gamma)\right|=2$, we define it to be the evaluation at the node of $\Sigma$ corresponding to $v$. If $\operatorname{val}_{v}(\Gamma) \leqslant 2$ and $\mathrm{E}_{v}(\Gamma)=\{e\}$, we take this homomorphism to be the evaluation at the preimage $x_{e, v} \in \Sigma_{e}$ of $P_{\vartheta(v)}$. If $v \in \mathrm{V}_{\mathbb{R}}^{\sigma}(\Gamma)$, the previous paragraph implies that the homomorphism

$$
H^{0}\left(\Sigma ; u^{*} \mathcal{L}_{n ; \mathbf{a}}\right)^{\tilde{\phi}_{n ; \mathbf{a}}} \longrightarrow\left(\left.\mathcal{L}_{n ; \mathbf{a}}\right|_{P_{\vartheta(v)}}\right)^{\tilde{\phi}_{n ; \mathbf{a}}}, \quad \xi \longrightarrow \widetilde{\mathrm{ev}}_{v}(\xi),
$$

is surjective. If $v \in \mathrm{V}_{\mathbb{C}}^{\sigma}(\Gamma)$, it implies that the homomorphism

$$
H^{0}\left(\Sigma ; u^{*} \mathcal{L}_{n ; \mathbf{a}}\right)^{\tilde{\phi}_{n ; \mathbf{a}}} \longrightarrow\left(\left.\left.\mathcal{L}_{n ; \mathbf{a}}\right|_{P_{\vartheta(v)}} \oplus \mathcal{L}_{n ; \mathbf{a}}\right|_{\left.P_{\phi(\vartheta(v)}\right)}\right)^{\tilde{\phi}_{n ; \mathbf{a}}}, \quad \xi \longrightarrow\left(\widetilde{\mathrm{ev}}_{v}(\xi), \widetilde{\mathrm{ev}}_{\sigma(v)}(\xi)\right),
$$

is surjective. From this, we obtain the following observation.

Lemma 5.2. Suppose $n=2 m+1$ and $\Gamma \in \overline{\mathcal{A}}_{g, l}(n, d)$. If $\vartheta(v)=n$ for some $v \in$ Ver, then

$$
\left.\mathbf{e}\left(\mathcal{V}_{n ; \mathbf{a}}^{\widetilde{\phi}_{n ; \mathbf{a}}}\right)\right|_{\overline{\mathcal{Z}}_{\Gamma, \sigma}}=0 \text {. }
$$

Proof. Since $n \notin 2 \mathbb{Z}, k>0$ and so the targets in (5.5) and (5.6) are non-trivial. If $v \in \mathrm{V}_{\mathbb{R}}^{\sigma}(\Gamma)$, the surjectivity of (5.5) implies that $\left.\mathcal{V}_{n ; \mathbf{a}}^{\widetilde{\phi}_{n ; \mathbf{a}}}\right|_{\mathcal{Z}_{\Gamma, \sigma}}$ contains a trivial real line bundle with the trivial $\mathbb{T}^{m}$-action. If $v \in \mathrm{V}_{\mathbb{C}}^{\sigma}(\Gamma)$, the surjectivity of (5.6) implies that $\left.\mathcal{V}_{n ; \mathbf{a}}^{\tilde{\phi}_{n ; \mathbf{a}}}\right|_{\mathcal{Z}_{\Gamma, \sigma}}$ contains a trivial complex line bundle with the trivial $\mathbb{T}^{m}$-action. In either case, $\left.\mathbf{e}\left(\mathcal{V}_{n ; \mathbf{a}}^{\tilde{\phi}_{n}}\right)\right|_{\mathcal{Z}_{\Gamma, \sigma}}$ vanishes.

By Lemma 5.2, $\overline{\mathcal{Z}}_{\Gamma, \sigma}$ does not contribute to (4.26) unless $\vartheta(v) \neq 2 m+1$ for all vertices $v \in$ Ver. Thus, it is sufficient to restrict attention to the subset

$$
\overline{\mathcal{A}}_{g, l}^{\prime}(n, d) \subset \overline{\mathcal{A}}_{g, l}(n, d)
$$

of pairs $(\Gamma, \sigma)$ satisfying the second condition in (4.15).

\subsection{The fixed-locus contribution}

For each pair $(\Gamma, \sigma)$ in $\overline{\mathcal{A}}_{g, l}^{\prime}(n, d)$, we will next describe the moduli spaces associated with the vertices and edges of $\Gamma$ and then determine the normal bundle to the $\mathbb{T}^{m}$-fixed locus $\overline{\mathcal{Z}}_{\Gamma, \sigma}$, after capping with $\mathbf{e}\left(\mathcal{V}_{n ; \mathbf{a}}^{\widetilde{\phi}_{n ; \mathbf{a}}}\right)$ if $k \in \mathbb{Z}^{+}$. We fix an element of $\overline{\mathcal{A}}_{g, l}^{\prime}(n, d)$ with $\Gamma$ as in (4.12) throughout this section.

Let $g_{0} \in \mathbb{Z}^{\geqslant 0}$. We will call a two-component symmetric surface $(\Sigma, \sigma)$ of the form

$$
\Sigma \equiv \Sigma_{1} \sqcup \Sigma_{2} \equiv\{1\} \times \Sigma_{0} \sqcup\{2\} \times \bar{\Sigma}_{0}, \quad \sigma(i, z)=(3-i, z) \forall(i, z) \in \Sigma,
$$

where $\Sigma_{0}$ is a connected oriented, possibly nodal, genus $g_{0}$ surface and $\bar{\Sigma}_{0}$ denotes $\Sigma_{0}$ with the opposite orientation, a nodal $g_{0}$-doublet. The arithmetic genus of a $g_{0}$-doublet is $2 g_{0}-1$. If $d_{0} \in \mathbb{Z}$ 
and $S_{1}$ and $S_{2}$ are finite sets with a fixed bijection $\sigma_{S}$ between them, let $\overline{\mathfrak{M}}_{2 g_{0}-1, S_{1} \sqcup S_{2}}^{\bullet}\left(\mathbb{P}^{n-1}, 2 d_{0}\right)^{\phi}$ denote the moduli space of stable real degree $2 d_{0} J_{0}$-holomorphic maps into $\mathbb{P}^{n-1}$ from nodal $g_{0}$-doublets with the first component carrying the $S_{1}$-marked points and with the marked points interchanged by the involution $\sigma_{S}$. We denote by $\overline{\mathfrak{M}}_{g_{0}, S_{1}}\left(\mathbb{P}^{n-1}, d_{0}\right)$ the usual moduli space of stable genus $g_{0} S_{1}$-marked degree $d_{0}$ maps into $\mathbb{P}^{n-1}$.

For each vertex $v$ of $\Gamma$, let $S_{v}$ be as in (4.17). Since $v \neq \sigma(v), \sigma$ induces an involution on the set $S_{v} \sqcup S_{\sigma(v)}$. If $\operatorname{val}_{v}(\Gamma) \geqslant 3$, let

$$
\overline{\mathfrak{M}}_{\Gamma ; v}=\overline{\mathfrak{M}}_{\mathfrak{g}(v), S_{v}}\left(\mathbb{P}^{n-1}, 0\right) \quad \text { and } \quad \overline{\mathfrak{M}}_{\Gamma ; v}^{\phi}=\overline{\mathfrak{M}}_{2 \mathfrak{g}(v)-1, S_{v} \sqcup S_{\sigma(v)}}^{\bullet}\left(\mathbb{P}^{n-1}, 0\right)^{\phi} .
$$

We denote by

$$
\mathrm{ev}_{v}: \overline{\mathfrak{M}}_{\Gamma ; v} \longrightarrow \mathbb{P}^{n-1} \quad \text { and } \quad \operatorname{ev}_{v}^{\phi}: \overline{\mathfrak{M}}_{\Gamma ; v}^{\phi} \longrightarrow \mathbb{P}^{n-1}
$$

the morphism sending each constant stable map to its value and the morphism sending each degree 0 holomorphic map from a doublet to its value on the first component, respectively. For each $e \in S_{v}$, let

$$
L_{v ; e} \longrightarrow \overline{\mathfrak{M}}_{\Gamma ; v} \quad \text { and } \quad L_{v ; e}^{\phi} \longrightarrow \overline{\mathfrak{M}}_{\Gamma ; v}^{\phi}
$$

be the universal tangent line bundles for this point.

The restriction of the map to the $S_{v}$-marked component induces a diffeomorphism

$$
\Psi_{\Gamma ; v}: \overline{\mathfrak{M}}_{\Gamma ; v}^{\phi} \longrightarrow \overline{\mathfrak{M}}_{\Gamma ; v}
$$

between the two moduli spaces which commutes with the evaluation morphisms (5.9) and naturally lifts to an isomorphism between the line bundles (5.10). Let

$$
\overline{\mathfrak{M}}_{\Gamma ; v}^{\phi ; \mathbb{T}}=\Psi_{\Gamma ; v}^{-1}\left(\overline{\mathfrak{M}}_{\Gamma ; v}^{\mathbb{T}}\right) \subset \overline{\mathfrak{M}}_{\Gamma ; v}^{\phi} ;
$$

see (5.3). We denote by

$$
\mathcal{N}_{\Gamma ; v} \longrightarrow \overline{\mathfrak{M}}_{\Gamma ; v}^{\mathbb{T}} \quad \text { and } \quad \mathcal{N}_{\Gamma ; v}^{\phi} \longrightarrow \overline{\mathfrak{M}}_{\Gamma ; v}^{\phi ; \mathbb{T}}
$$

the normal bundle of $\overline{\mathfrak{M}}_{\Gamma ; v}^{\mathbb{T}}$ in $\overline{\mathfrak{M}}_{\Gamma ; v}$ and of $\overline{\mathfrak{M}}_{\Gamma ; v}^{\phi ; \mathbb{T}}$ in $\overline{\mathfrak{M}}_{\Gamma ; v}^{\phi}$, respectively. The diffeomorphism (5.11) descends to an isomorphism from the second bundle to the first.

For each $e \in \operatorname{Edg}$ and $v \in e$, let

$$
S_{e, v}= \begin{cases}\{v\}, & \text { if } \operatorname{val}_{v}(\Gamma)+\left|\mathrm{E}_{v}(\Gamma)\right| \geqslant 4 \\ \mathfrak{m}^{-1}(v), & \text { if } \operatorname{val}_{v}(\Gamma)+\left|\mathrm{E}_{v}(\Gamma)\right|=3 \\ \varnothing, & \text { if } \operatorname{val}_{v}(\Gamma)+\left|\mathrm{E}_{v}(\Gamma)\right|=2\end{cases}
$$

If $e=\left\{v_{1}, v_{2}\right\}$, let $S_{e}=S_{e, v_{1}} \sqcup S_{e, v_{2}}$. If $e \in \mathrm{E}_{\mathbb{R}}^{\sigma}(\Gamma), S_{e}$ is either empty or consists of two elements interchanged by $\sigma$. In such a case, let

$$
\overline{\mathfrak{M}}_{\Gamma, \sigma ; e}^{\phi} \equiv \overline{\mathfrak{M}}_{0, S_{e}}\left(\mathbb{P}^{n-1}, \mathfrak{d}(e)\right)^{\phi}
$$




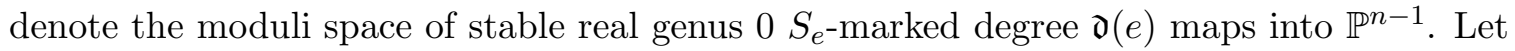

$$
\overline{\mathfrak{M}}_{\Gamma, \sigma ; e}^{\phi ; \mathbb{T}} \subset \overline{\mathfrak{M}}_{\Gamma, \sigma ; e}^{\phi}
$$

be the preimage of $\overline{\mathfrak{M}}_{\Gamma, \sigma ; e}^{\phi ; \mathbb{T} ; \circ}$ under the forgetful morphism

$$
\mathfrak{f}_{\Gamma, \sigma ; e}^{\phi}: \overline{\mathfrak{M}}_{\Gamma, \sigma ; e}^{\phi} \longrightarrow \overline{\mathfrak{M}}_{\Gamma, \sigma ; e}^{\phi ; \circ} \equiv \overline{\mathfrak{M}}_{0,0}\left(\mathbb{P}^{n-1}, \mathfrak{d}(e)\right)^{\phi} ;
$$

see (5.2). We denote by

$$
\mathcal{N}_{\Gamma, \sigma ; e}^{\phi} \longrightarrow \overline{\mathfrak{M}}_{\Gamma, \sigma ; e}^{\phi ; \mathbb{T}}
$$

the normal bundle of $\overline{\mathfrak{M}}_{\Gamma, \sigma ; e}^{\phi ; \mathbb{T}}$ in $\overline{\mathfrak{M}}_{\Gamma, \sigma ; e}^{\phi}$.

If $e \in \mathrm{E}_{\mathbb{C}}^{\sigma}(\Gamma), S_{e}$ and $S_{\sigma(e)}$ consist of either one or two elements each; the involution $\sigma$ interchanges the two sets. Let

$$
\overline{\mathfrak{M}}_{\Gamma ; e}=\overline{\mathfrak{M}}_{0, S_{e}}\left(\mathbb{P}^{n-1}, \mathfrak{d}(e)\right) \quad \text { and } \quad \overline{\mathfrak{M}}_{\Gamma, \sigma ; e}^{\phi}=\overline{\mathfrak{M}}_{-1, S_{e} \sqcup S_{\sigma(e)}}\left(\mathbb{P}^{n-1}, 2 \mathfrak{d}(e)\right)^{\phi} .
$$

Thus, $\overline{\mathfrak{M}}_{\Gamma, \sigma ; e}^{\phi}=\overline{\mathfrak{M}}_{\Gamma, \sigma ; \sigma(e)}^{\phi}$ and the restriction of the map to the $S_{e}$-marked component induces a diffeomorphism

$$
\Psi_{\Gamma, \sigma ; e}: \overline{\mathfrak{M}}_{\Gamma, \sigma ; e}^{\phi} \longrightarrow \overline{\mathfrak{M}}_{\Gamma ; e}
$$

between the two moduli spaces. Let

$$
\overline{\mathfrak{M}}_{\Gamma ; e}^{\mathbb{T}} \subset \overline{\mathfrak{M}}_{\Gamma ; e}
$$

be the preimage of $\overline{\mathfrak{M}}_{\Gamma ; e}^{\mathbb{T} ; 0}$ under the forgetful morphism

$$
\mathfrak{f}_{\Gamma ; e}: \overline{\mathfrak{M}}_{\Gamma ; e} \longrightarrow \overline{\mathfrak{M}}_{\Gamma ; e}^{\circ} \equiv \overline{\mathfrak{M}}_{0,0}\left(\mathbb{P}^{n-1}, \mathfrak{d}(e)\right)^{\phi}
$$

and define

$$
\overline{\mathfrak{M}}_{\Gamma, \sigma ; e}^{\phi ; \mathbb{T}}=\Psi_{\Gamma ; v}^{-1}\left(\overline{\mathfrak{M}}_{\Gamma ; e}^{\mathbb{T}}\right) \subset \overline{\mathfrak{M}}_{\Gamma, \sigma ; e}^{\phi} .
$$

We denote by

$$
\mathcal{N}_{\Gamma ; e} \longrightarrow \overline{\mathfrak{M}}_{\Gamma ; e}^{\mathbb{T}} \quad \text { and } \quad \mathcal{N}_{\Gamma, \sigma ; e}^{\phi} \longrightarrow \overline{\mathfrak{M}}_{\Gamma, \sigma ; e}^{\phi ; \mathbb{T}}
$$

the normal bundle of $\overline{\mathfrak{M}}_{\Gamma ; e}^{\mathbb{T}}$ in $\overline{\mathfrak{M}}_{\Gamma ; e}$ and of $\overline{\mathfrak{M}}_{\Gamma, \sigma ; e}^{\phi ; \mathbb{T}}$ in $\overline{\mathfrak{M}}_{\Gamma, \sigma ; e}^{\phi}$, respectively. The diffeomorphism (5.12) descends to an isomorphism from the second bundle to the first.

In either of the two cases above, for $v \in S_{e} \cup S_{\sigma(e)}$ let

$$
\mathrm{ev}_{e, v}^{\phi}: \overline{\mathfrak{M}}_{\Gamma, \sigma ; e}^{\phi} \longrightarrow \mathbb{P}^{n-1} \quad \text { and } \quad L_{e ; v}^{\phi} \longrightarrow \overline{\mathfrak{M}}_{\Gamma, \sigma ; e}^{\phi}
$$

be the evaluation morphism and the universal tangent line bundle for the marked point indexed by $v$. If $e \in \mathrm{E}_{\mathbb{C}}^{\sigma}(\Gamma)$ and $v \in S_{e}$, let

$$
\mathrm{ev}_{e, v}: \overline{\mathfrak{M}}_{\Gamma ; e} \longrightarrow \mathbb{P}^{n-1} \quad \text { and } \quad L_{e ; v} \longrightarrow \overline{\mathfrak{M}}_{\Gamma ; e}
$$

be the analogous objects for the target space of the diffeomorphism (5.12). We extend these definitions to $v \in S_{\sigma(e)}$ by setting

$$
\mathrm{ev}_{e, v}=\phi \circ \mathrm{ev}_{e, \sigma(v)} \quad \text { and } \quad L_{e ; v}=\overline{L_{e ; \sigma(v)}} .
$$


The diffeomorphism (5.12) commutes with the evaluation morphisms and naturally lifts to an isomorphism between the universal tangent line bundles.

Choose $\mathrm{V}_{+}^{\sigma}(\Gamma) \subset$ Ver and $\mathrm{E}_{+}^{\sigma}(\Gamma) \subset$ Edg satisfying (4.24) and (4.25). Let

$$
\mathrm{V}_{+; 3}^{\sigma}(\Gamma)=\left\{v \in \mathrm{V}_{+}^{\sigma}(\Gamma): \operatorname{val}_{v}(\Gamma) \geqslant 3\right\}, \quad \mathrm{V}_{+; 2}^{\sigma}(\Gamma)=\left\{v \in \mathrm{V}_{+}^{\sigma}(\Gamma): \operatorname{val}_{v}(\Gamma),\left|\mathrm{E}_{v}(\Gamma)\right|=2\right\} .
$$

We define

$$
\begin{aligned}
\widetilde{\mathcal{Z}}_{\Gamma, \sigma} & \equiv \prod_{v \in \mathrm{V}_{+; 3}^{\sigma}(\Gamma)} \overline{\mathfrak{M}}_{\Gamma ; v}^{\phi ; \mathbb{T}} \times \underset{e \in \mathrm{E}_{\mathbb{R}}^{\sigma}(\Gamma) \sqcup \mathrm{E}_{+}^{\sigma}(\Gamma)}{\overline{\mathfrak{M}}_{\Gamma, \sigma ; e}^{\phi ; \mathbb{T}}} \\
& \approx \prod_{v \in \mathrm{V}_{+; 3}^{\sigma}(\Gamma)} \overline{\mathcal{M}}_{\mathfrak{g}(v) ; S_{v}} \times \prod_{e \in \mathrm{E}_{\mathbb{R}}^{\sigma}(\Gamma)} \overline{\mathfrak{M}}_{\Gamma, \sigma ; e}^{\phi ; \mathbb{T}} \times \prod_{e \in \mathrm{E}_{+}^{\sigma}(\Gamma)} \overline{\mathfrak{M}}_{\Gamma ; e}^{\mathbb{T}}
\end{aligned}
$$

The fixed locus $\overline{\mathcal{Z}}_{\Gamma, \sigma}$ corresponding to $(\Gamma, \sigma)$ is then given by

$$
\overline{\mathcal{Z}}_{\Gamma, \sigma}=\widetilde{\mathcal{Z}}_{\Gamma, \sigma} / \operatorname{Aut}(\Gamma, \sigma)
$$

with the group $\operatorname{Aut}(\Gamma, \sigma)$ acting trivially. For example, in the case of the pair $(\Gamma, \sigma)$ represented by the first diagram in Figure 1

$$
\mathcal{Z}_{\Gamma, \sigma}=\overline{\mathcal{Z}}_{\Gamma, \sigma} \approx\left(\overline{\mathcal{M}}_{0,3} \times \overline{\mathcal{M}}_{0,3}\right) / S_{3} \approx\{\mathrm{pt}\} / S_{3} \subset \overline{\mathfrak{M}}_{2,0}\left(\mathbb{P}^{n-1}, 3\right)^{\phi},
$$

with the symmetric group $S_{3}$ acting trivially.

For each $e \in \mathrm{Edg}$, define

$$
e_{\bullet} \in \mathrm{E}_{\mathbb{R}}^{\sigma}(\Gamma) \cup \mathrm{E}_{+}^{\sigma}(\Gamma) \quad \text { by } \quad e_{\bullet} \in\{e, \sigma(e)\} .
$$

Let

$$
\begin{gathered}
\overline{\mathfrak{M}}_{\Gamma}^{\phi}=\prod_{v \in \mathrm{V}_{+; 3}^{\sigma}(\Gamma)} \overline{\mathfrak{M}}_{\Gamma ; v}^{\phi} \times \prod_{e \in \mathrm{E}_{\mathbb{R}}^{\sigma}(\Gamma) \cup \mathrm{E}_{+}^{\sigma}(\Gamma)} \overline{\mathfrak{M}}_{\Gamma, \sigma ; e}^{\phi}, \\
\overline{\mathfrak{M}}_{\Gamma}^{\phi}=\left\{\left(\left(\mathbf{u}_{v}\right)_{v \in \mathrm{V}_{+; 3}^{\sigma}(\Gamma)},\left(\mathbf{u}_{e}\right)_{e \in \mathrm{E}_{\mathbb{R}}^{\sigma}(\Gamma) \sqcup \mathrm{E}_{+}^{\sigma}(\Gamma)}\right) \in \overline{\mathfrak{M}}_{\Gamma}^{\phi}: \operatorname{ev}_{v}\left(\mathbf{u}_{v}\right)=\operatorname{ev}_{e_{\bullet}, v}\left(\mathbf{u}_{e}\right) \forall v \in \mathrm{V}_{+; 3}^{\sigma}(\Gamma), e \in \mathrm{E}_{v}(\Gamma),\right. \\
\left.\quad \operatorname{ev}_{e_{1} \bullet v}\left(\mathbf{u}_{e_{1} \bullet}\right)=\operatorname{ev}_{e_{2}, v}\left(\mathbf{u}_{e_{2} \bullet}\right) \forall v \in \mathrm{V}_{+; 2}^{\sigma}(\Gamma), e_{1}, e_{2} \in \mathrm{E}_{v}(\Gamma)\right\} .
\end{gathered}
$$

For each $v \in \mathrm{V}_{+; 3}^{\sigma}(\Gamma) \cup \mathrm{V}_{+; 2}^{\sigma}(\Gamma)$, there is thus a well-defined evaluation morphism

$$
\mathrm{ev}_{v}: \overline{\mathfrak{M}}_{\Gamma}^{\phi} \longrightarrow \mathbb{P}^{n-1}, \quad \operatorname{ev}_{v}\left(\left(\mathbf{u}_{v^{\prime}}\right)_{v^{\prime} \in \mathrm{V}_{+; 3}^{\sigma}(\Gamma)},\left(\mathbf{u}_{e}\right)_{e \in \mathrm{E}_{\mathbb{R}}^{\sigma}(\Gamma) \sqcup \mathrm{E}_{+}^{\sigma}(\Gamma)}\right)=\operatorname{ev}_{e_{\bullet}, v}\left(\mathbf{u}_{e}\right) \text { if } e \in \mathrm{E}_{v}(\Gamma) .
$$

For $v \in \mathrm{V}_{+; 3}^{\sigma}(\Gamma)$ and $e \in \mathrm{E}_{\mathbb{R}}^{\sigma}(\Gamma) \sqcup \mathrm{E}_{+}^{\sigma}(\Gamma)$, let

$$
\pi_{v}: \overline{\mathfrak{M}}_{\Gamma}^{\phi} \longrightarrow \overline{\mathfrak{M}}_{\Gamma ; v}^{\phi} \quad \text { and } \quad \pi_{e}: \overline{\mathfrak{M}}_{\Gamma}^{\phi} \longrightarrow \overline{\mathfrak{M}}_{\Gamma, \sigma ; e}^{\phi}
$$

be the projection maps. 
Define

$$
\begin{aligned}
& L_{\Gamma}=\bigoplus_{v \in \mathrm{V}_{+; 3}^{\sigma}(\Gamma)} \bigoplus_{e \in \mathrm{E}_{v}(\Gamma)} \pi_{v}^{*} L_{v ; e}^{\phi} \otimes \pi_{e_{\bullet}}^{*} L_{e_{\bullet} ; v}^{\phi} \oplus \underset{v \in \mathrm{V}_{+; 2}^{\sigma}(\Gamma)}{\bigoplus} \underset{e \in \mathrm{E}_{v}(\Gamma)}{\bigotimes} \pi_{e_{\bullet}}^{*} L_{e_{\bullet} ; v}^{\phi}, \\
& \mathcal{N}_{\Gamma} \mathbb{P}=\underset{v \in \mathrm{V}_{+; 3}^{\sigma}(\Gamma)}{\bigoplus}\left(\mathrm{ev}_{v}^{*} T \mathbb{P}^{n-1}\right)^{\{v\} \sqcup \mathrm{E}_{v}(\Gamma)} \oplus \underset{v \in \mathrm{V}_{+; 2}^{\sigma}(\Gamma)}{\bigoplus}\left(\operatorname{ev}_{v}^{*} T \mathbb{P}^{n-1}\right)^{\mathrm{E}_{v}(\Gamma)}, \\
& \mathcal{L}_{\Gamma}=\underset{v \in \mathrm{V}_{+; 3}^{\sigma}(\Gamma)}{\bigoplus}\left(\mathrm{ev}_{v}^{*} \mathcal{L}_{n ; \mathbf{a}}\right)^{\{v\} \sqcup \mathrm{E}_{v}(\Gamma)} \oplus \underset{v \in \mathrm{V}_{+; 2}^{\sigma}(\Gamma)}{\bigoplus}\left(\mathrm{ev}_{v}^{*} \mathcal{L}_{n ; \mathbf{a}}\right)^{\mathrm{E}_{v}(\Gamma)} .
\end{aligned}
$$

For $v \in \mathrm{V}_{+; 3}^{\sigma}(\Gamma) \cup \mathrm{V}_{+; 2}^{\sigma}(\Gamma)$, denote by

$$
\begin{aligned}
& \mathcal{N}_{\Gamma ; v}^{\Delta} \mathbb{P} \subset\left\{\begin{array}{ll}
\left(\operatorname{ev}_{v}^{*} T \mathbb{P}^{n-1}\right)^{\{v\}} \mathrm{E}_{v}(\Gamma), & \text { if } v \in \mathrm{V}_{+; 3}^{\sigma}(\Gamma) ; \\
\left(\operatorname{ev}_{v}^{*} T \mathbb{P}^{n-1}\right)^{\mathrm{E}_{v}(\Gamma)}, & \text { if } v \in \mathrm{V}_{+; 2}^{\sigma}(\Gamma) ;
\end{array} \quad\right. \text { and } \\
& \mathcal{L}_{\Gamma ; v}^{\Delta} \subset \begin{cases}\left(\mathcal{L}_{n ; \mathbf{a}}\right)^{\{v\} \sqcup \mathrm{E}_{v}(\Gamma),} & \text { if } v \in \mathrm{V}_{+; 3}^{\sigma}(\Gamma) ; \\
\left(\mathcal{L}_{n ; \mathbf{a}}\right)^{\mathrm{E}_{v}(\Gamma)}, & \text { if } v \in \mathrm{V}_{+; 2}^{\sigma}(\Gamma) ;\end{cases}
\end{aligned}
$$

the small diagonals (all components are the same). Let $\mathcal{N}_{\Gamma}^{\prime} \mathbb{P}$ and $\mathcal{L}_{\Gamma}^{\prime}$ be the quotients of $\mathcal{N}_{\Gamma} \mathbb{P}$ and $\mathcal{L}_{\Gamma}$ by the subbundles

$$
\mathcal{N}_{\Gamma}^{\Delta} \mathbb{P}=\underset{v \in \mathrm{V}_{+; 3}^{\sigma}(\Gamma) \cup \mathrm{V}_{+; 2}^{\sigma}(\Gamma)}{\bigoplus \mathcal{N}_{\Gamma ; v}^{\Delta} \mathbb{P}} \quad \text { and } \quad \mathcal{L}_{\Gamma}^{\Delta}=\underset{v \in \mathrm{V}_{+; 3}^{\sigma}(\Gamma) \cup \mathrm{V}_{+; 2}^{\sigma}(\Gamma)}{\bigoplus} \mathcal{L}_{\Gamma ; v}^{\Delta}
$$

respectively. Since the vector bundles $L_{\Gamma}, \mathcal{N}_{\Gamma}^{\prime} \mathbb{P}$, and $\mathcal{L}_{\Gamma}^{\prime}$ are complex, they are canonically oriented. The differentials of the evaluation morphisms $\mathrm{ev}_{v}$ and $\mathrm{ev}_{e_{\bullet}, v}$ induce a homomorphism

$$
\widetilde{\mathrm{ev}}_{\Gamma}^{\mathbb{P}}:\left.\left(T \overline{\mathfrak{M}}_{\Gamma}^{\phi}\right)^{\mathrm{vrt}}\right|_{\overline{\mathfrak{M}}_{\Gamma}^{\prime}} \longrightarrow \mathcal{N}_{\Gamma}^{\prime} \mathbb{P} .
$$

The latter descends to an isomorphism

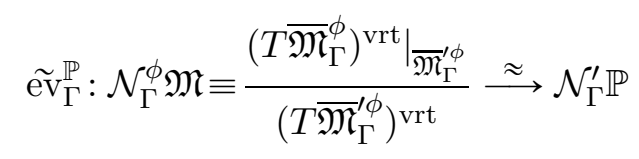

from the normal bundle of $\overline{\mathfrak{M}}_{\Gamma}^{\phi}$ in $\overline{\mathfrak{M}}_{\Gamma}^{\phi}$. The natural bundle homomorphisms

$$
\mathcal{V}_{n ; \mathbf{a}}^{\tilde{\phi}_{n ; \mathbf{a}}} \longrightarrow \mathrm{ev}_{v}^{*} \mathcal{L}_{n ; \mathbf{a}} \quad \text { and } \quad \mathcal{V}_{n ; \mathbf{a}}^{\tilde{\phi}_{n ; \mathbf{a}}} \longrightarrow \mathrm{ev}_{e \bullet, v}^{*} \mathcal{L}_{n ; \mathbf{a}}
$$

over $\overline{\mathfrak{M}}_{\Gamma ; v}^{\phi}$ and $\overline{\mathfrak{M}}_{\Gamma, \sigma ; e}^{\phi}$, respectively, given by the evaluations at the marked points similarly induce a bundle homomorphism

$$
\widetilde{\mathrm{ev}}_{\Gamma}^{\mathcal{L}}: \mathcal{V}_{\Gamma, \sigma} \equiv \bigoplus_{v \in \mathrm{V}_{+; 3}^{\sigma}(\Gamma)}\left(\pi_{v}^{*} \mathcal{V}_{n ; \mathbf{a}}^{\widetilde{\phi}_{n ; \mathbf{a}}} \oplus \underset{e \in \mathrm{E}_{v}(\Gamma)}{\bigoplus} \pi_{e .}^{*} \mathcal{V}_{n ; \mathbf{a}}^{\widetilde{\phi}_{n} \mathbf{a}}\right) \oplus \underset{v \in \mathrm{V}_{+; 2}^{\sigma}(\Gamma)}{\bigoplus} \underset{e \in \mathrm{E}_{v}(\Gamma)}{\bigoplus} \pi_{e}^{*} \mathcal{V}_{n ; \mathbf{a}}^{\widetilde{\phi}_{n ;}} \longrightarrow \mathcal{L}_{\Gamma}^{\prime}
$$

over $\overline{\mathfrak{M}}_{\Gamma}^{\phi}$. 
Denote by

$$
\iota_{\Gamma}: \overline{\mathfrak{M}}_{\Gamma}^{\prime \phi} \longrightarrow \overline{\mathfrak{M}}_{g, l}\left(\mathbb{P}^{n-1}, d\right)^{\phi}
$$

the natural node-identifying immersion which sends $\widetilde{\mathcal{Z}}_{\Gamma, \sigma}$ to $\overline{\mathcal{Z}}_{\Gamma, \sigma}$. For each $v \in \mathrm{V}_{+; 3}^{\sigma}(\Gamma)$ and $e \in \mathrm{E}_{v}(\Gamma)$, it identifies the marked point of $\mathbf{u}_{v}$ indexed by $e$ with the marked point of $\mathbf{u}_{e}$. indexed by $v$. For each $v \in \mathrm{V}_{+; 2}^{\sigma}(\Gamma)$ and $\mathrm{E}_{v}(\Gamma)=\left\{e_{1}, e_{2}\right\}, \iota_{\Gamma}$ identifies the marked points of $\mathbf{u}_{e_{1}}$. and $\mathbf{u}_{e_{2}}$. indexed by $v$. There is a natural isomorphism

$$
\mathcal{N} \iota_{\Gamma} \equiv \frac{\iota_{\Gamma}^{*}\left(T \overline{\mathfrak{M}}_{g, l}\left(\mathbb{P}^{n-1}, d\right)^{\phi}\right)^{\mathrm{vrt}}}{\left(T \overline{\mathfrak{M}}_{\Gamma}^{\prime \phi}\right)^{\mathrm{vrt}}} \approx L_{\Gamma}
$$

of vector bundles over $\overline{\mathfrak{M}}_{\Gamma}^{\prime \phi}$. Since the exact sequence

$$
0 \longrightarrow \iota_{\Gamma}^{*} \mathcal{V}_{n ; \mathbf{a}}^{\widetilde{\phi}_{n ; \mathbf{a}}} \longrightarrow \mathcal{V}_{\Gamma, \sigma} \stackrel{\widetilde{\mathrm{ev}}_{\Gamma}^{\mathcal{L}}}{\longrightarrow} \mathcal{L}_{\Gamma}^{\prime} \longrightarrow 0
$$

of vector bundles over $\overline{\mathfrak{M}}_{\Gamma}^{\phi}$ is $\mathbb{T}^{m}$-equivariant,

$$
\iota_{\Gamma}^{*} \mathbf{e}\left(\mathcal{V}_{n ; \mathbf{a}}^{\tilde{\phi}_{n ; \mathbf{a}}}\right)=\frac{1}{\mathbf{e}\left(\mathcal{L}_{\Gamma}^{\prime}\right)} \mathbf{e}\left(\mathcal{V}_{\Gamma, \sigma}\right)
$$

For each $v \in$ Ver, the elements of the subset $\mathfrak{m}^{-1}(v) \subset S_{v}$ carry signs as elements of $S_{l}$. We decorate the elements in the complement $\mathrm{E}_{v}(\Gamma) \subset S_{v}$ of $\mathfrak{m}^{-1}(v)$ with the plus sign if $v \in \mathrm{V}_{+}^{\sigma}(\Gamma)$ and with the minus sign if $\sigma(v) \in \mathrm{V}_{+}^{\sigma}(\Gamma)$. The involution $\sigma$ on $S_{v} \sqcup S_{\sigma(v)}$ then interchanges the two subsets and changes the sign of each element. If $e \in \mathrm{Edg}$ and $v \in S_{e} \cap$ Ver, we decorate $v \in S_{e}$ with the plus sign if $v \in \mathrm{V}_{+}^{\sigma}(\Gamma)$ and with the minus sign if $\sigma(v) \in \mathrm{V}_{+}^{\sigma}(\Gamma)$. If $e \in \mathrm{E}_{\mathbb{R}}^{\sigma}(\Gamma), \sigma$ induces an involution on $S_{e}$ that changes the sign of each element. If $e \in \mathrm{E}_{\mathbb{C}}^{\sigma}(\Gamma)$, the involution $\sigma$ on $S_{e} \sqcup S_{\sigma(e)}$ then interchanges the two subsets and changes the sign of each element. Let $S_{e}^{-} \subset S_{e}$ be the subset of negatively marked elements. In particular,

$$
\left|S_{e}^{-}\right|=1 \quad \forall e \in \mathrm{E}_{\mathbb{R}}^{\sigma}(\Gamma), \quad\left|S_{e}^{-}\right| \in\{0,1,2\} \quad \forall e \in \mathrm{E}_{\mathbb{C}}^{\sigma}(\Gamma) ;
$$

all three possibilities in the last case are in general attainable.

Denote by

$$
\tilde{\mathcal{N}}_{\Gamma, \sigma}^{\phi} \equiv \bigoplus_{v \in \mathrm{V}_{+; 3}^{\sigma}(\Gamma)}\left(\pi_{v}^{*} \mathcal{N}_{\Gamma ; v}^{\phi} \oplus \underset{e \in \mathrm{E}_{v}(\Gamma)}{\bigoplus_{e}} \pi_{\bullet}^{*} \mathcal{N}_{\Gamma, \sigma ; e_{\bullet}}^{\phi}\right) \oplus \underset{v \in \mathrm{V}_{+; 2}^{\sigma}(\Gamma)}{\bigoplus} \bigoplus_{e \in \mathrm{E}_{v}(\Gamma)} \pi_{e}^{*} \mathcal{N}_{\Gamma, \sigma ; e_{\bullet}}^{\phi} \longrightarrow \widetilde{\mathcal{Z}}_{\Gamma, \sigma}
$$

the normal bundle of $\widetilde{\mathcal{Z}}_{\Gamma, \sigma}$ in $\overline{\mathfrak{M}}_{\Gamma}^{\phi}$. Under the assumptions on $(\Gamma, \sigma)$ in Theorem 4.6,

$$
\widetilde{\mathcal{Z}}_{\Gamma, \sigma} \subset \mathfrak{M}_{g, l}^{\star}\left(\mathbb{P}^{n-1}, d\right)^{\phi} ;
$$

see (4.2) for the notation. The real orientation on $\left(X_{n ; \mathbf{a}}, \phi_{n ; \mathbf{a}}\right)$ described in Section 2.3 determines an orientation on the moduli space $\overline{\mathfrak{M}}_{g, l}\left(X_{n ; \mathbf{a}}, d\right)^{\phi_{n ; \mathbf{a}}}$. Analogously to [28, Section 5.3], it also determines orientations on the homology classes

$$
\frac{\mathbf{e}\left(\mathcal{V}_{n ; \mathbf{a}}^{\widetilde{\phi}_{n ; \mathbf{a}}}\right)}{\mathbf{e}\left(\mathcal{N}_{\Gamma, \sigma}^{\phi}\right)} \cap\left[\overline{\mathcal{Z}}_{\Gamma, \sigma}\right]^{\mathrm{vrt}} \quad \text { and } \quad \frac{\mathbf{e}\left(\mathcal{V}_{\Gamma, \sigma}\right)}{\mathbf{e}\left(\widetilde{\mathcal{N}}_{\Gamma, \sigma}^{\phi}\right)} \cap\left[\widetilde{\mathcal{Z}}_{\Gamma, \sigma}\right]^{\mathrm{vrt}}
$$


that correspond to the orientations of the two moduli spaces of real maps into $\left(X_{n ; \mathbf{a}}, \omega_{n ; \mathbf{a}}, \phi_{n ; \mathbf{a}}\right)$ via the section $s_{n ; \mathbf{a}}$. By (5.18), (5.19), and (5.20),

$$
\iota_{\Gamma}^{*}\left(\frac{\mathbf{e}\left(\mathcal{V}_{n ; \mathbf{a}}^{\widetilde{\phi}_{n}}\right)}{\mathbf{e}\left(\mathcal{N}_{\Gamma, \sigma}^{\phi}\right)}\right)=\left(\frac{\mathbf{e}\left(\mathcal{N}_{\Gamma}^{\prime} \mathbb{P}\right)}{\mathbf{e}\left(\mathcal{L}_{\Gamma}^{\prime}\right)}\right) \frac{1}{\mathbf{e}\left(L_{\Gamma}\right)}\left(\frac{\mathbf{e}\left(\mathcal{V}_{\Gamma, \sigma}\right)}{\mathbf{e}\left(\tilde{\mathcal{N}}_{\Gamma, \sigma}^{\phi}\right)}\right) .
$$

Since the orientations on the two classes in (5.21) arise from the same real orientation on $\left(X_{n ; \mathbf{a}}, \phi_{n ; \mathbf{a}}\right)$, [12, Theorem 1.2] provides for a comparison between the two classes in (5.21). Since the section $s_{n ; \mathbf{a}}$ is holomorphic, the leading fraction on the right-hand side of (5.22) corresponds to the Euler class of $T X_{n ; \mathbf{a}}$ at the chosen node in each pair of the conjugate nodes considered for the purposes of [12, Theorem 1.2]. Since the number of such pairs is

$$
\operatorname{nd}(\Gamma) \equiv \sum_{v \in \mathrm{V}_{+; 3}^{\sigma}(\Gamma)}\left|\mathrm{E}_{v}(\Gamma)\right|+\left|\mathrm{V}_{+; 2}^{\sigma}(\Gamma)\right|
$$

we find that

$$
\frac{\mathbf{e}\left(\mathcal{V}_{n ; \mathbf{a}}^{\tilde{\phi}_{n ; \mathbf{a}}}\right)}{\mathbf{e}\left(\mathcal{N}_{\Gamma, \sigma}^{\phi}\right)} \cap\left[\overline{\mathcal{Z}}_{\Gamma, \sigma}\right]^{\mathrm{vrt}}=\frac{(-1)^{\mathrm{nd}(\Gamma)}}{|\operatorname{Aut}(\Gamma, \sigma)|}\left(\frac{\mathbf{e}\left(\mathcal{N}_{\Gamma}^{\prime} \mathbb{P}\right)}{\mathbf{e}\left(\mathcal{L}_{\Gamma}^{\prime}\right)}\right) \iota_{\Gamma *}\left(\frac{1}{\mathbf{e}\left(L_{\Gamma}\right)}\left(\frac{\mathbf{e}\left(\mathcal{V}_{\Gamma, \sigma}\right)}{\mathbf{e}\left(\tilde{\mathcal{N}}_{\Gamma, \sigma}^{\phi}\right)}\right) \cap\left[\widetilde{\mathcal{Z}}_{\Gamma, \sigma}\right]^{\mathrm{vrt}}\right) .
$$

The automorphism factor above is the degree of the covering

$$
\iota_{\Gamma}: \widetilde{\mathcal{Z}}_{\Gamma, \sigma} \longrightarrow \overline{\mathcal{Z}}_{\Gamma, \sigma}
$$

it corresponds to the first factor in (4.26). By definition of $\tilde{\mathcal{N}}_{\Gamma, \sigma}^{\phi}, \mathcal{V}_{\Gamma, \sigma}$, and $\widetilde{\mathcal{Z}}_{\Gamma, \sigma}$,

$$
\begin{aligned}
\left(\frac{\mathbf{e}\left(\mathcal{V}_{\Gamma, \sigma}\right)}{\mathbf{e}\left(\widetilde{\mathcal{N}}_{\Gamma, \sigma}^{\phi}\right)}\right) \cap\left[\widetilde{\mathcal{Z}}_{\Gamma, \sigma}\right]^{\mathrm{vrt}}= & \prod_{v \in \mathrm{V}_{+; 3}^{\sigma}(\Gamma)}\left(\left(\frac{\mathbf{e}\left(\mathcal{V}_{n ; \mathbf{a}}^{\widetilde{\phi}_{n ; \mathbf{a}}}\right)}{\mathbf{e}\left(\mathcal{N}_{\Gamma ; v}^{\phi}\right)}\right) \cap\left[\overline{\mathfrak{M}}_{\Gamma ; v}^{\phi ; T}\right]^{\mathrm{vrt}}\right) \\
& \times \prod_{e \in \mathrm{E}_{\mathbb{R}}^{\sigma}(\Gamma) \sqcup \mathrm{E}_{+}^{\sigma}(\Gamma)}\left(\left(\frac{\mathbf{e}\left(\mathcal{V}_{n ; \mathbf{a}}^{\phi_{n}}\right)}{\mathbf{e}\left(\mathcal{N}_{\Gamma, \sigma ; e}^{\phi}\right)}\right) \cap\left[\overline{\mathfrak{M}}_{\Gamma, \sigma ; e}^{\phi ; \mathbb{T}}\right]^{\mathrm{vrt}}\right)
\end{aligned}
$$

under the decomposition (5.14). The identities (5.24) and (5.25) nearly split the equivariant contribution of $(\Gamma, \sigma)$ to (4.4), i.e. the left-hand side of (5.24), into contributions from the components in (5.14). The exceptional term $\mathbf{e}\left(L_{\Gamma}\right)$ splits into products of Euler classes of $\mathbb{T}^{m}$-equivariant line bundles, each of which involves only two components in (5.14). We will associate each factor of $\mathbf{e}\left(L_{\Gamma}\right)$ with the corresponding vertex $v$ in $\mathrm{V}_{+; 3}^{\sigma}(\Gamma)$ or in $\mathrm{V}_{+; 2}^{\sigma}(\Gamma)$.

\subsection{The edge and vertex contributions}

It remains to compute the cap products in (5.25) and to determine the factors of $\mathbf{e}\left(L_{\Gamma}\right)$. The first case of Lemma 5.3 below justifies the alternative case of the fixed-edge contribution (4.23), i.e. when it vanishes. The second case of this lemma justifies the first restriction in (4.15) and in particular rules out contributions from the families $\overline{\mathfrak{M}}_{\Gamma, \sigma ; e}^{\phi ; T ; \circ}$ of real conics of Remark 5.1. Lemma 5.4 expresses the contributions from the families $\overline{\mathfrak{M}}_{\Gamma ; e}^{\mathbb{T} ; \circ}$ of complex conics in terms of (4.21). The crucial 
fixed-edge contribution (4.23) arises from Proposition 5.5. Lemmas 5.3 and 5.4 are proved at the end of this section; Proposition 5.5 is established in Section 5.4. Analogously to (4.1), let

$$
\pi_{n ; \mathbf{a}}: \mathcal{V}_{n ; \mathbf{a}}=\overline{\mathfrak{M}}_{g, l}\left(\mathcal{L}_{n ; \mathbf{a}}, d\right) \longrightarrow \overline{\mathfrak{M}}_{g, l}\left(\mathbb{P}^{n-1}, d\right)
$$

Lemma 5.3. Suppose $\Gamma \in \overline{\mathcal{A}}_{g, l}^{\prime}(n, d)$ and $\mathrm{E}_{\mathbb{R}}^{\sigma}(\Gamma) \neq \varnothing$. If either $a_{i} \in 2 \mathbb{Z}$ for some $i \in[k]$ or $\mathfrak{d}(e) \in 2 \mathbb{Z}$ for some $e \in \mathrm{E}_{\mathbb{R}}^{\sigma}(\Gamma)$, then

$$
\iota_{*}\left(\frac{\mathbf{e}\left(\mathcal{V}_{n ; \mathbf{a}}^{\tilde{\phi}_{n ; \mathbf{a}}}\right)}{\mathbf{e}\left(\mathcal{N}_{\Gamma, \sigma}^{\phi}\right)} \cap\left[\overline{\mathcal{Z}}_{\Gamma, \sigma}\right]^{\mathrm{vrt}}\right)=0 \in \mathcal{H}_{*}^{\mathbb{T}^{m}}\left(\overline{\mathfrak{M}}_{g, l}\left(\mathbb{P}^{n-1}, d\right)^{\phi}\right)
$$

where $\iota: \mathcal{Z}_{\Gamma, \sigma} \longrightarrow \overline{\mathfrak{M}}_{g, l}\left(\mathbb{P}^{n-1}, d\right)^{\phi}$ is the inclusion of the fixed locus.

Lemma 5.4. Suppose $\Gamma \in \overline{\mathcal{A}}_{g, l}^{\prime}(n, d)$, e $\in \mathrm{E}_{\mathbb{C}}^{\sigma}(\Gamma)$, 4.20) is satisfied, and $\mathbf{b} \in\left(\mathbb{Z}^{\geqslant 0}\right)^{e}$. Then,

$$
\begin{aligned}
\left(\prod_{v \in e} c_{1}\left(L_{e ; v}\right)^{\mathbf{b}(v)}\right. & \left.\frac{\mathbf{e}\left(\mathcal{V}_{n ; \mathbf{a}}\right)}{\mathbf{e}\left(\mathcal{N}_{\Gamma ; e}\right)}\right) \cap\left[\overline{\mathfrak{M}}_{\Gamma ; e}^{\mathbb{T}}\right] \\
& =-\left(\prod_{v \in e}\left(-\psi_{e ; v}\right)^{\mathbf{b}(v)-\left|S_{e, v}\right|}\right) \cdot \begin{cases}{[\mathrm{RHS} \text { of (4.21) }],} & \text { if } k=1 ; \\
0, & \text { if } k \geqslant 2 .\end{cases}
\end{aligned}
$$

Proposition 5.5. Suppose $\Gamma \in \overline{\mathcal{A}}_{g, l}^{\prime}(n, d)$ and $e \in \mathrm{E}_{\mathbb{R}}^{\sigma}(\Gamma)$. If $a_{i} \notin 2 \mathbb{Z}$ for all $i \in[k]$ and $\mathfrak{d}(e) \notin 2 \mathbb{Z}$, then

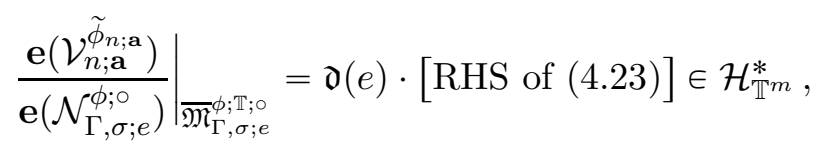

with $\overline{\mathfrak{M}}_{\Gamma, \sigma ; e}^{\phi ; \mathbb{T} ; \circ}$ as on the first line in (5.2).

Proof of Theorem 4.6. By Lemmas 5.2 and 5.3, all nonzero $\mathbb{T}^{m}$-equivariant contributions to (4.4) come from the $\mathbb{T}^{m}$-fixed loci $\overline{\mathcal{Z}}_{\Gamma, \sigma}$ corresponding to the admissible pairs $(\Gamma, \sigma)$, i.e. the elements of the collection $\mathcal{A}_{g, l}(n, d)$ defined by (4.15). Furthermore, if a fixed locus $\overline{\mathcal{Z}}_{\Gamma, \sigma}$ contributes to (4.4), then $\mathrm{E}_{\mathbb{R}}^{\sigma}(\Gamma)=\varnothing$ if $a_{i} \in 2 \mathbb{Z}$ for some $i \in[k]$ and $k=1$ if some $e \in \mathrm{E}_{+}^{\sigma}(\Gamma)$ satisfies (4.20). From now on, we assume that $(\Gamma, \sigma)$ is an admissible pair satisfying these two conditions.

By (5.16), (5.17), and the definitions of $\mathcal{N}_{\Gamma}^{\prime} \mathbb{P}$ and $\mathcal{L}_{\Gamma}^{\prime}$ just below,

$$
\left.\frac{\mathbf{e}\left(\mathcal{N}_{\Gamma}^{\prime} \mathbb{P}\right)}{\mathbf{e}\left(\mathcal{L}_{\Gamma}^{\prime}\right)}\right|_{\tilde{\mathcal{Z}}_{\Gamma, \sigma}}=\prod_{v \in \mathrm{V}_{+; 3}^{\sigma}(\Gamma)}\left(\frac{\mathbf{e}\left(T_{P_{\vartheta(v)}} \mathbb{P}^{n-1}\right)}{\langle\mathbf{a}\rangle \alpha_{\vartheta(v)}^{k}}\right)^{\left|\mathrm{E}_{v}(\Gamma)\right|} \cdot \prod_{v \in \mathrm{V}_{+; 2}^{\sigma}(\Gamma)}\left(\frac{\mathbf{e}\left(T_{P_{\vartheta(v)}} \mathbb{P}^{n-1}\right)}{\langle\mathbf{a}\rangle \alpha_{\vartheta(v)}^{k}}\right) .
$$

By (5.15),

$$
\begin{aligned}
& \left.\mathbf{e}\left(L_{\Gamma}\right)\right|_{\tilde{\mathcal{Z}}_{\Gamma, \sigma}}=\prod_{v \in \mathrm{V}_{+; 3}^{\sigma}(\Gamma)} \prod_{e \in \mathrm{E}_{v}(\Gamma)}\left(c_{1}\left(L_{e \bullet ; v}^{\phi}\right)+c_{1}\left(L_{v ; e}^{\phi}\right)\right) \cdot \prod_{v \in \mathrm{V}_{+; 2}^{\sigma}(\Gamma)}\left(-\sum_{e \in \mathrm{E}_{v}(\Gamma)} \psi_{e_{\bullet} ; v}\right), \\
& =\prod_{v \in \mathrm{V}_{+; 3}^{\sigma}(\Gamma)} \Psi_{\Gamma ; v}^{*} \prod_{e \in \mathrm{E}_{v}(\Gamma)}\left(-\psi_{e ; v}-\pi_{v}^{*} \psi_{e}\right) \cdot \prod_{v \in \mathrm{V}_{+; 2}^{\sigma}(\Gamma)}\left(-\sum_{e \in \mathrm{E}_{v}(\Gamma)} \psi_{e ; v}\right) .
\end{aligned}
$$


The right-hand sides of (5.28) and (5.29) are elements of $\mathcal{H}_{\mathbb{T}^{m}}^{*}$ and $\mathcal{H}_{\mathbb{T}^{m}}^{*} \otimes H^{*}\left(\widetilde{\mathcal{Z}}_{\Gamma, \sigma}\right)$, respectively. By [12, Theorem 1.4],

$$
\begin{gathered}
\Psi_{\Gamma ; v *}\left(\left(\frac{\mathbf{e}\left(\mathcal{V}_{n ; \mathbf{a}}^{\tilde{\phi}_{n ; \mathbf{a}}}\right)}{\mathbf{e}\left(\mathcal{N}_{\Gamma ; v}^{\phi}\right)}\right) \cap\left[\overline{\mathfrak{M}}_{\Gamma ; v}^{\phi ; \mathbb{T}}\right]^{\mathrm{vrt}}\right)=(-1)^{\mathfrak{g}(v)-1+\left|S_{v}^{-}\right|}\left(\frac{\mathbf{e}\left(\mathcal{V}_{n ; \mathbf{a}}\right)}{\mathbf{e}\left(\mathcal{N}_{\Gamma ; v}\right)}\right) \cap\left[\overline{\mathfrak{M}}_{\Gamma ; v}^{\mathbb{T}}\right]^{\mathrm{vrt}}, \\
\Psi_{\Gamma, \sigma ; e *}\left(\left(\frac{\mathbf{e}\left(\mathcal{V}_{n ; \mathbf{a}}^{\widetilde{\phi}_{n ; \mathbf{a}}}\right)}{\mathbf{e}\left(\mathcal{N}_{\Gamma, \sigma ; e}^{\phi}\right)}\right) \cap\left[\overline{\mathfrak{M}}_{\Gamma, \sigma ; e}^{\phi ; \mathbb{T}}\right]^{\mathrm{vrt}}\right)=(-1)^{-1+\left|S_{e}^{-}\right|}\left(\frac{\mathbf{e}\left(\mathcal{V}_{n ; \mathbf{a}}\right)}{\mathbf{e}\left(\mathcal{N}_{\Gamma ; e}\right)}\right) \cap\left[\overline{\mathfrak{M}}_{\Gamma ; e}^{\mathbb{T}}\right],
\end{gathered}
$$

for all $v \in \mathrm{V}_{+; 3}^{\sigma}(\Gamma)$ and $e \in \mathrm{E}_{+}^{\sigma}(\Gamma)$; the right-hand sides above carry standard complex orientations. Since

$$
\left.\iota_{\Gamma}^{*}\left(\psi_{i}^{b_{i}}, \mathrm{ev}_{i}^{*} \mathbf{x}^{p_{i}}\right)\right|_{\tilde{\mathcal{Z}}_{\Gamma, \sigma}}= \begin{cases}\left(\pi_{v}^{*} \Psi_{\Gamma ; v}^{*} \psi_{i}^{p_{i}}, \alpha_{\vartheta(v)}^{p_{i}}\right), & \text { if } i^{+} \in S_{v} \\ \left(\left(-\pi_{v}^{*} \Psi_{\Gamma ; v}^{*} \psi_{i}\right)^{b_{i}},\left(-\alpha_{\vartheta(v)}\right)^{p_{i}}\right), & \text { if } i^{-} \in S_{v}\end{cases}
$$

the first statement in (5.30) and [18, Section 27.6] give

$$
\begin{aligned}
& \left(\frac{\prod_{\substack{1 \leqslant i \leqslant l \\
i \pm \in S_{v}}}\left(\psi_{i}^{b_{i}} \mathrm{ev}_{i}^{*} \mathbf{x}^{p_{i}}\right)}{\Psi_{\Gamma ; v \in \mathrm{E}_{v}(\Gamma)}^{*} \prod_{e ; v}\left(-\psi_{e ; v}-\pi_{v}^{*} \psi_{e}\right)} \frac{\mathbf{e}\left(\mathcal{V}_{n ; \mathbf{a}}^{\phi_{\phi}}\right)}{\mathbf{e}\left(\mathcal{N}_{\Gamma ; v}^{\phi}\right)}\right) \cap\left[\overline{\mathfrak{M}}_{\Gamma ; v}^{\phi ; \mathbb{T}}\right]^{\mathrm{vrt}} \\
& =(-1)^{\mathfrak{s}_{v}-\left|\mathrm{E}_{v}(\Gamma)\right|}\left(\frac{\mathbf{e}\left(T_{P_{\vartheta(v)}} \mathbb{P}^{n-1}\right)}{\langle\mathbf{a}\rangle \alpha_{\vartheta(v)}^{k}}\right)^{-1} \alpha_{\vartheta(v)}^{|\mathbf{p}|_{v}} \int_{\overline{\mathcal{M}}_{\mathfrak{g}(v), S_{v}}} \frac{\mathbf{e}\left(\mathbb{E}^{*} \otimes T_{P_{\vartheta(v)}} \mathbb{P}^{n-1}\right)}{\prod_{e \in \mathrm{E}_{v}(\Gamma)}\left(-\psi_{e ; v}-\psi_{e}\right)} \prod_{\substack{1 \leqslant i \leqslant l \\
i \pm \in S_{v}}} \psi_{i}^{b_{i}}
\end{aligned}
$$

for all $v \in \mathrm{V}_{+; 3}^{\sigma}(\Gamma)$.

For any $e \in \operatorname{Edg}$ and $v \in e$, let

$$
|\mathbf{b}|_{e, v}=\sum_{\substack{1 \leqslant i \leqslant l \\ i^{ \pm} \in S_{e, v}}} b_{i}, \quad|\mathbf{b}|_{e, v}^{-}=\sum_{\substack{1 \leqslant i \leqslant l \\ i^{-} \in S_{e, v}}} b_{i}, \quad|\mathbf{p}|_{e, v}=\sum_{\substack{1 \leqslant i \leqslant l \\ i^{ \pm} \in S_{e, v}}} p_{i}, \quad|\mathbf{p}|_{e, v}^{-}=\sum_{\substack{1 \leqslant i \leqslant l \\ i^{-} \in S_{e, v}}} p_{i}
$$

Since $S_{e, v} \cap[l]$ consists of at most one element,

$$
\left.\iota_{\Gamma}^{*} \operatorname{ev}_{i}^{*} \mathbf{x}^{p_{i}}\right|_{\widetilde{\mathcal{Z}}_{\Gamma, \sigma}}=(-1)^{|\mathbf{p}|_{e, v}^{-}} \alpha_{\vartheta(v)}^{|\mathbf{p}|_{e, v}} \quad \forall i \in S_{e, v} \cap[l] .
$$

If $e \in \mathrm{E}_{+}^{\sigma}(\Gamma)$ does not satisfy (4.20), then

$$
\frac{\mathbf{e}\left(\mathcal{V}_{n ; \mathbf{a}}\right)}{\mathbf{e}\left(\mathcal{N}_{\Gamma ; e}\right)}=\left(\prod_{v \in e}\left(-\psi_{e ; v}\right)^{\left|S_{e, v}\right|}\right)^{-1} \mathfrak{f}_{\Gamma ; e}^{*}\left(\frac{\mathbf{e}\left(\mathcal{V}_{n ; \mathbf{a}}\right)}{\mathbf{e}\left(\mathcal{N}_{\Gamma ; e}^{\circ}\right)}\right),\left.\quad \iota_{\Gamma}^{*} \psi_{i}^{b_{i}}\right|_{\tilde{\mathcal{Z}}_{\Gamma, \sigma}}=(-1)^{|\mathbf{b}|_{e, v}^{-}} \psi_{e ; v}^{|\mathbf{b}|_{e, v}} .
$$

The second statement in (5.30) and [18, Sections 27.2,27.6] thus give

$$
\begin{aligned}
& \left(\prod_{\substack{1 \leqslant i \leqslant l \\
i \pm \in S_{e}}}\left(\psi_{i}^{b_{i}} \operatorname{ev}_{i}^{*} \mathbf{x}^{p_{i}}\right) \frac{\mathbf{e}\left(\mathcal{V}_{n ; \mathbf{a}}^{\widetilde{\phi}_{n ;}}\right)}{\mathbf{e}\left(\mathcal{N}_{\Gamma, \sigma ; e}^{\phi}\right)}\right) \cap\left[\overline{\mathfrak{M}}_{\Gamma, \sigma ; e}^{\phi ; \mathbb{T}}\right]^{\mathrm{vrt}} \\
& =(-1)^{\left|S_{e}^{-}\right|} \prod_{v \in e} \frac{\prod_{\substack{i_{ \pm} \leqslant i \leqslant l \\
i_{e, v}}}(-1)^{|\mathbf{b}|_{e, v}^{-}+|\mathbf{p}|_{e, v}^{-}} \psi_{e ; v}^{|\mathbf{b}|_{e, v}} \alpha_{\vartheta(v)}^{|\mathbf{p}|_{e, v}}}{\left(-\psi_{e ; v}\right)^{\left|S_{e, v}\right|}}[\operatorname{RHS} \text { of (4.22) }]
\end{aligned}
$$


for all $e \in \mathrm{E}_{+}^{\sigma}(\Gamma)$ not satisfying (4.20).

If $e \in \mathrm{E}_{+}^{\sigma}(\Gamma)$ satisfies (4.20), then

$$
\left.\iota_{\Gamma}^{*} \psi_{i}^{b_{i}}\right|_{\tilde{\mathcal{Z}}_{\Gamma, \sigma}}=(-1)^{|\mathbf{b}|_{e, v}^{-}}\left(-c_{1}\left(L_{e ; v}\right)\right)^{|\mathbf{b}|_{e, v}} .
$$

The second statement in (5.30), Lemma 5.4, and (5.32) thus give

$$
\begin{aligned}
& \left(\prod_{\substack{1 \leqslant i \leqslant l \\
i \pm \in S_{e}}}\left(\psi_{i}^{b_{i}} \operatorname{ev}_{i}^{*} \mathbf{x}^{p_{i}}\right) \frac{\mathbf{e}\left(\mathcal{V}_{n ; \mathbf{a}}^{\tilde{\phi}_{n ; \mathbf{a}}}\right)}{\mathbf{e}\left(\mathcal{N}_{\Gamma, \sigma ; e}^{\phi}\right)}\right) \cap\left[\overline{\mathfrak{M}}_{\Gamma, \sigma ; e}^{\phi ; \mathbb{T}}\right]^{\mathrm{vrt}} \\
& =(-1)^{\left|S_{e}^{-}\right|} \prod_{v \in e} \frac{\prod_{\substack{1 \leqslant i \leqslant l \\
i^{ \pm} \in S_{e, v}}}(-1)^{|\mathbf{b}|_{e, v}^{-}+|\mathbf{p}|_{e, v}^{-}} \psi_{e ; v}^{|\mathbf{b}|_{e, v}} \alpha_{\vartheta(v)}^{|\mathbf{p}|_{e, v}}}{\left(-\psi_{e ; v}\right)^{\left|S_{e, v}\right|}}[\text { RHS of (4.21) }]
\end{aligned}
$$

for all $e \in \mathrm{E}_{+}^{\sigma}(\Gamma)$ satisfying (4.20).

If $e \in \mathrm{E}_{\mathbb{R}}^{\sigma}(\Gamma)$, then precisely one of the vertices $v_{e} \in e$ belongs to $\mathrm{V}_{+}^{\sigma}(\Gamma)$ and

$$
\left.\iota_{\Gamma}^{*} \mathrm{ev}_{i}^{*} \mathbf{x}^{p_{i}}\right|_{\tilde{\mathcal{Z}}_{\Gamma, \sigma}}=(-1)^{|\mathbf{p}|_{e, v_{e}}^{-}} \alpha_{\vartheta\left(v_{e}\right)}^{|\mathbf{p}|_{e, v_{e}}},\left.\quad \iota_{\Gamma}^{*} \psi_{i}^{b_{i}}\right|_{\widetilde{\mathcal{Z}}_{\Gamma, \sigma}}=(-1)^{|\mathbf{b}|_{e, v_{e}}^{-}} \psi_{e ; v_{e}}^{|\mathbf{b}|_{e, v_{e}}} .
$$

Since $\overline{\mathfrak{M}}_{\Gamma, \sigma ; e}^{\phi ; \mathbb{T}}$ consists of a single point with the automorphism group $\mathbb{Z}_{\mathfrak{d}(e)}$, Proposition $[5.5$ thus gives

$$
\begin{aligned}
& \left(\prod_{\substack{1 \leqslant i \leqslant l \\
i \pm \in S_{e}}}\left(\psi_{i}^{b_{i}} \mathrm{ev}_{i}^{*} \mathbf{x}^{p_{i}}\right) \frac{\mathbf{e}\left(\mathcal{V}_{n ; \mathbf{a}}^{\tilde{\phi}_{n ; \mathbf{a}}}\right)}{\mathbf{e}\left(\mathcal{N}_{\Gamma, \sigma ; e}^{\phi}\right)}\right) \cap\left[\overline{\mathfrak{M}}_{\Gamma, \sigma ; e}^{\phi ; \mathbb{T}}\right]^{\mathrm{vrt}} \\
& =\frac{\prod_{\substack{1 \leqslant i \leqslant l \\
i^{ \pm} \in S_{e, v}}}(-1)^{|\mathbf{b}|_{e, v_{e}}^{-}+|\mathbf{p}|_{e, v_{e}}^{-}} \psi_{e ; v_{e}}^{|\mathbf{b}|_{e, v_{e}}} \alpha_{\vartheta\left(v_{e}\right)}^{|\mathbf{p}|_{e, v_{e}}}}{\left(-\psi_{e ; v_{e}}\right)^{\left|S_{e, v_{e}}\right|}}[\text { RHS of }(\underline{4.23})]
\end{aligned}
$$

for all $e \in \mathrm{E}_{\mathbb{R}}^{\sigma}(\Gamma)$.

By (5.24), (5.25), and (5.29), the left-hand side of (4.26) is the product of the leading fraction in (5.24) with the right-hand sides of (5.28), (5.31), (5.33), (5.34), and (5.35). The factor on the right-hand side of (4.26) corresponding to a vertex $v \in \mathrm{V}_{+; 3}^{\sigma}(\Gamma)$ is the product of

- the corresponding factor on RHS of (5.28),

- RHS of (5.31),

- the non-edge factors on RHSs of (5.33) and (5.34) corresponding to either $v \in e$ or $\sigma(v) \in e$,

- the non-edge factors on RHS of (5.35) with $v_{e}=e$, and

- $(-1)$ to the power of the summand $\left|\mathrm{E}_{v}(\Gamma)\right|$ in (5.23). 
The factor on the right-hand side of (4.26) corresponding to a vertex $v \in \mathrm{V}_{+; 2}^{\sigma}(\Gamma)$ is obtained similarly, except the product of the contributions from the second and last bullets above is replaced by

$$
\left(-\sum_{e \in \mathrm{E}_{v}(\Gamma)} \psi_{e ; v}\right)^{-1} \cdot(-1)^{1} .
$$

The role of (4.18) now is played by (4.19) with

$$
\operatorname{val}_{v}(\Gamma),\left|\mathrm{E}_{v}(\Gamma)\right|=2, \quad|\mathbf{b}|_{v},|\mathbf{p}|_{v}=0 .
$$

There is no contribution associated with $v \in \mathrm{V}_{+}^{\sigma}(\Gamma)$ such that $\operatorname{val}_{v}(\Gamma)=1$; this is consistent with

$$
(-1)^{\mathfrak{s}_{v}}[\text { RHS of (4.19) }]=1
$$

in this case. For $v \in \mathrm{V}_{+}^{\sigma}(\Gamma)$ such that $\operatorname{val}_{v}(\Gamma)=2$ and $\left|\mathrm{E}_{v}(\Gamma)\right|=1$, there is a unique edge $e$ in $\mathrm{E}_{\mathbb{R}}^{\sigma}(\Gamma) \cup$ $\mathrm{E}_{+}^{\sigma}(\Gamma)$ containing either $v$ or $\sigma(v)$. The factor on the right-hand side of (4.26) corresponding to such $v$ is the non-edge factor on the right-hand side of either (5.33), (5.34), or (5.35) corresponding to the associated flag $(e, v)$. This is the only case for which the numbers $|\mathbf{b}|_{e, v}^{-}$and $|\mathbf{p}|_{e, v}^{-}$may be nonzero.

Proof of Lemma 5.3. Suppose $e \in \mathrm{E}_{\mathbb{R}}^{\sigma}(\Gamma)$. If $k \in \mathbb{Z}^{+}$and either $a_{i} \in 2 \mathbb{Z}$ for some $i \in[k]$ or $\mathfrak{d}(e) \in 2 \mathbb{Z}$, then

$$
\mathcal{V}_{n ; \mathbf{a}}^{\widetilde{\phi}_{n} \mathbf{a}} \longrightarrow \overline{\mathfrak{M}}_{\Gamma, \sigma ; e}^{\phi}
$$

contains a subbundle of odd rank and thus $\mathbf{e}\left(\mathcal{V}_{n ; \mathbf{a}}^{\widetilde{\phi}_{n ; \mathbf{a}}}\right)=0$. In the first case, this is the subbundle associated with the $i$-th factor in the vector bundle $\mathcal{L}_{n ; \mathbf{a}}$. In the second case, the subbundle associated with every factor in $\mathcal{L}_{n ; \mathbf{a}}$ has odd rank.

It remains to consider the case $\mathfrak{d}(e) \in 2 \mathbb{Z}$ and $k=0$. The latter implies that $X_{n ; \mathbf{a}}=\mathbb{P}^{2 m-1}$. If $\phi=\eta_{2 m}$, then

$$
\overline{\mathfrak{M}}_{\Gamma, \sigma ; e}^{\phi ; \mathbb{T}} \subset \overline{\mathfrak{M}}_{\Gamma, \sigma ; e}^{\phi} \equiv \overline{\mathfrak{M}}_{0, S_{e}}\left(\mathbb{P}^{2 m-1}, \mathfrak{d}(e)\right)^{\phi}=\varnothing ;
$$

see [3, Lemma 1.9]. Suppose $\phi=\tau_{2 m}^{\prime}$. The set $\overline{\mathfrak{M}}_{\Gamma, \sigma ; e}^{\phi ; \mathbb{T}}$ then consists of two equivalence classes of real maps: one with the standard involution $\tau=\tau_{2}$ on the domain and the other with the fixed-point-free involution $\eta=\eta_{2}$. We denote the associated uncompactified moduli spaces of real maps by

$$
\mathfrak{M}_{\Gamma, \sigma ; e}^{\phi, \tau ; \mathbb{T}} \subset \mathfrak{M}_{\Gamma, \sigma ; e}^{\phi, \tau} \quad \text { and } \quad \mathfrak{M}_{\Gamma, \sigma ; e}^{\phi, \eta ; \mathbb{T}} \subset \mathfrak{M}_{\Gamma, \sigma ; e}^{\phi, \eta}
$$

respectively.

A real orientation on $\left(\mathbb{P}^{2 m-1}, \tau_{2 m}\right)$ directly determines orientations on $\mathfrak{M}_{\Gamma, \sigma ; e}^{\phi, \tau}$ and $\mathfrak{M}_{\Gamma, \sigma ; e}^{\phi, \eta}$; see 11 , Corollary 5.10]. In order to extend some set of orientations across the common boundary of these two moduli spaces, we reverse the orientation of the second moduli space; see the end of [11, Section 3.2]. Thus, it is sufficient to show that

$$
\left.\mathbf{e}\left(\mathcal{N}_{\Gamma, \sigma ; e}^{\phi}\right)\right|_{\mathfrak{M}_{\Gamma, \sigma ; e}^{\phi, \tau ; \mathbb{T}}}=\left.\mathbf{e}\left(\mathcal{N}_{\Gamma, \sigma ; e}^{\phi}\right)\right|_{\mathfrak{M}_{\Gamma, \sigma ; e}^{\phi, \eta ; \mathbb{T}}} \in \mathcal{H}_{\mathbb{T}^{m}}^{*}
$$

before the orientation reversal. Since adding a pair of conjugate points has the same effect on the two sides of (5.36) , we can assume that $S_{e}$ consists of a pair of conjugate points and the value of $\vartheta$ 
on the positive vertex is some $i \in \mathbb{Z}^{+}-2 \mathbb{Z}$.

By [3, Proposition 5.5], the algebraic orientations on $\mathfrak{M}_{\Gamma, \sigma ; e}^{\phi, \tau}$ and $\mathfrak{M}_{\Gamma, \sigma ; e}^{\phi, \eta}$ defined in [3, Section 5.2] also do not extend across the common boundary. By [3, Lemma 5.1], the moduli space $\overline{\mathfrak{M}}_{\Gamma, \sigma ; e}^{\phi}$ is connected. Thus, the algebraic orientations on $\mathfrak{M}_{\Gamma, \sigma ; e}^{\phi, \tau}$ and $\mathfrak{M}_{\Gamma, \sigma ; e}^{\phi, \eta}$ are either both the same or both opposite of the orientations induced by a real orientation on $\left(\mathbb{P}^{2 m-1}, \tau_{2 m}\right)$. In either case, it is sufficient to establish (5.36) for the algebraic orientations on the two moduli spaces. By [3, Remark 6.9], both classes in (5.36) are then the negative of the right-hand side of [3, (6.6)] with $d_{0}=\mathfrak{d}(e)$ and $\lambda_{i}=\alpha_{i}$. In particular, they are equal.

Remark 5.6. Suppose $\mathfrak{d}(e) \in 2 \mathbb{Z}$ as above. If $m \in 2 \mathbb{Z}$, the orientation on $\mathfrak{M}_{\Gamma, \sigma ; e}^{\phi, \tau}$ induced by the canonical real orientation of Section 2.3 is the same as the orientation induced by the canonical spin structure of [3, Section 5.5]; see [12, Theorem 1.5]. The two classes in (5.36) with respect to the canonical real orientation (and before the orientation reversal) are thus given by [3, (6.6)]. If $m \notin 2 \mathbb{Z}$, the orientation on $\mathfrak{M}_{\Gamma, \sigma ; e}^{\phi, \tau}$ induced by the canonical real orientation of Section 2.3 is the same as the orientation induced by the associated relative spin structure; see [12, Theorem 1.5]. The latter is the same as the orientation induced by the relative spin structure of [3, Remark 6.5]. By the beginning of [3, Remark 6.9] and the middle of the preceding paragraph in [3], the two classes in (5.36) with respect to the canonical real orientation (and before the orientation reversal) are thus again given by [3, (6.6)].

Proof of Lemma 5.4. We use the $\mathbb{T}^{2}$-action on $\mathbb{P}_{\vartheta\left(v_{1}\right), \vartheta\left(v_{2}\right), n}^{2}$ induced by the $\mathbb{T}^{2}$-action on $\mathbb{C}^{3}$ with weights $\alpha_{\vartheta\left(v_{1}\right)}, \alpha_{\vartheta\left(v_{2}\right)}$, and 0 and set $\alpha_{\vartheta\left(v_{1}\right)}=-\alpha_{\vartheta\left(v_{2}\right)}$ after computing the equivariant contribution to the left-hand side of (5.26) from the fixed loci of this action on $\overline{\mathfrak{M}}_{\Gamma ; e}^{\mathbb{T}}$.

The fixed locus of the $\mathbb{T}^{2}$-action on $\overline{\mathfrak{M}}_{\Gamma ; e}^{\mathbb{T}}$ consists of two points: the $\mathbb{T}^{2}$-invariant degree $\mathfrak{d}(e)$ cover of the line $\mathbb{P}_{\vartheta\left(v_{1}\right), \vartheta\left(v_{2}\right)}^{1}$ and the $\mathbb{T}^{2}$-invariant degree $\mathfrak{d}(e) / 2$ cover of $\mathbb{P}_{\vartheta\left(v_{1}\right), n}^{1} \cup \mathbb{P}_{\vartheta\left(v_{2}\right), n}^{1}$. Let

$$
\mathcal{Z}_{\Gamma ; e}^{\prime \mathbb{T}}, \mathcal{Z}_{\Gamma^{\prime} ; e}^{\mathbb{T}} \subset \overline{\mathfrak{M}}_{\Gamma ; e}^{\mathbb{T}} \subset \overline{\mathfrak{M}}_{\Gamma ; e} \equiv \overline{\mathfrak{M}}_{0, S_{e}}\left(\mathbb{P}^{n-1}, \mathfrak{d}(e)\right)
$$

be the corresponding one-element subspaces. Denote by

$$
\mathcal{Z}_{\Gamma ; e}^{\prime \mathbb{T} ; \circ} \subset \overline{\mathfrak{M}}_{\Gamma ; e}^{\mathbb{T} ; \circ} \subset \overline{\mathfrak{M}}_{\Gamma ; e}^{\circ} \equiv \overline{\mathfrak{M}}_{0,0}\left(\mathbb{P}^{n-1}, \mathfrak{d}(e)\right)
$$

the image of $\mathcal{Z}_{\Gamma ; e}^{\prime T}$ under the forgetful morphism and by

$$
\mathcal{N} \mathcal{Z}_{\Gamma ; e}^{\prime \mathbb{T}}, \mathcal{N}_{\Gamma ; e}^{\prime} \longrightarrow \mathcal{Z}_{\Gamma ; e}^{\prime \mathbb{T}} \quad \text { and } \quad \mathcal{N}_{\Gamma ; e}^{\prime \mathbb{T} ; \circ}, \mathcal{N}_{\Gamma ; e}^{\prime \circ} \longrightarrow \mathcal{Z}_{\Gamma ; e}^{\prime \mathbb{T} ; \circ}
$$

the normal bundles in the intermediate subspaces and in the ambient moduli spaces, respectively.

By the proof of Lemma $\left[5.2,\left.\mathbf{e}\left(\mathcal{V}_{n ; \mathbf{a}}\right)\right|_{\mathcal{Z}_{\Gamma^{\prime} ; e}^{\mathbb{T}}}=0\right.$. From the classical Atiyah-Bott Localization Theorem [1], we thus find that

$$
\left(\prod_{v \in e} c_{1}\left(L_{e ; v}\right)^{\mathbf{b}(v)} \frac{\mathbf{e}\left(\mathcal{V}_{n ; \mathbf{a}}\right)}{\mathbf{e}\left(\mathcal{N}_{\Gamma ; e}\right)}\right) \cap\left[\overline{\mathfrak{M}}_{\Gamma ; e}^{\mathbb{T}}\right]=\left.\left(\prod_{v \in e} c_{1}\left(L_{e ; v}\right)^{\mathbf{b}(v)} \frac{\mathbf{e}\left(\mathcal{V}_{n ; \mathbf{a}}\right)}{\mathbf{e}\left(\mathcal{N}_{\Gamma ; e}\right)}\right)\right|_{\mathcal{Z}_{\Gamma ; e}^{\prime \mathbb{T}}} \cdot \frac{1}{\mathbf{e}\left(\mathcal{N} \mathcal{Z}_{\Gamma ; e}^{\prime \mathbb{T}}\right)}
$$


with respect to the specified $\mathbb{T}^{2}$-action.

By Exercise 27.2.4 and (27.8) in [18],

$$
\begin{aligned}
& \left(\frac{1}{\mathbf{e}\left(\mathcal{N} \mathcal{Z}_{\Gamma ; e}^{\prime \mathbb{T} ; \circ}\right)} \frac{\mathbf{e}\left(\mathcal{V}_{n ; \mathbf{a}}\right)}{\mathbf{e}\left(\mathcal{N}_{\Gamma ; e}^{\circ}\right)}\right) \cap\left[\mathcal{Z}_{\Gamma ; e}^{\prime \mathbb{T} ; \circ}\right]=\left(\frac{\mathbf{e}\left(\mathcal{V}_{n ; \mathbf{a}}\right)}{\mathbf{e}\left(\mathcal{N}_{\Gamma ; e}^{\prime \circ}\right)}\right) \cap\left[\mathcal{Z}_{\Gamma ; e}^{\prime \mathbb{T} ; \circ}\right] \\
& =-\frac{\langle\mathbf{a}\rangle}{\mathfrak{d}(e)(\mathfrak{d}(e) !)^{2}} \cdot \frac{\left(\frac{\alpha_{\vartheta\left(v_{1}\right)}+\alpha_{\vartheta\left(v_{2}\right)}}{2}\right)^{k-1}}{\prod_{\substack{1 \leqslant j<n \\
j \neq \vartheta\left(v_{1}\right), \vartheta\left(v_{2}\right)}}\left(\frac{\alpha_{\vartheta\left(v_{1}\right)}+\alpha_{\vartheta\left(v_{2}\right)}}{2}-\alpha_{j}\right)} \\
& \times \frac{\prod_{i=1}^{k} \prod_{r=1}^{a_{i} \mathfrak{d}(e) / 2}\left(a_{i}^{2}\left(\frac{\alpha_{\vartheta\left(v_{1}\right)}+\alpha_{\vartheta\left(v_{2}\right)}}{2}\right)^{2}-r^{2}\left(\frac{\alpha_{\vartheta\left(v_{1}\right)}-\alpha_{\vartheta\left(v_{2}\right)}}{\mathfrak{d}(e)}\right)^{2}\right)}{\left(\frac{\alpha_{\vartheta\left(v_{1}\right)}-\alpha_{\vartheta\left(v_{2}\right)}}{\mathfrak{d}(d)}\right)^{2 \mathfrak{d}(e)-2} \prod_{j \neq \vartheta\left(v_{1}\right), \vartheta\left(v_{2}\right)} \prod_{r=1}^{\mathfrak{d}(e) / 2}\left(\left(\frac{\alpha_{\vartheta\left(v_{1}\right)}+\alpha_{\vartheta\left(v_{2}\right)}}{2}-\alpha_{j}\right)^{2}-r^{2}\left(\frac{\alpha_{\vartheta\left(v_{1}\right)}-\alpha_{\vartheta\left(v_{2}\right)}}{\mathfrak{d}(e)}\right)^{2}\right)}
\end{aligned}
$$

with $\alpha_{n} \equiv 0$. For $\alpha_{\vartheta\left(v_{1}\right)}=-\alpha_{\vartheta\left(v_{2}\right)}$, the right-hand side of this expression reduces to the negative of the right-hand side of (4.21) if $k=1$ and to 0 if $k \geqslant 2$. Since

$$
\mathbf{e}\left(\mathcal{N} \mathcal{Z}_{\Gamma ; e}^{\prime \mathbb{T}}\right)=\mathfrak{f}_{\Gamma ; e}^{*} \mathbf{e}\left(\mathcal{N} \mathcal{Z}_{\Gamma ; e}^{\prime \mathbb{T} ; \circ}\right), \quad \frac{\mathbf{e}\left(\mathcal{V}_{n ; \mathbf{a}}\right)}{\mathbf{e}\left(\mathcal{N}_{\Gamma ; e}\right)}=\left(\prod_{v \in e} c_{1}\left(L_{e ; v}\right)^{\left|S_{e, v}\right|}\right)^{-1} \mathfrak{f}_{\Gamma ; e}^{*}\left(\frac{\mathbf{e}\left(\mathcal{V}_{n ; \mathbf{a}}\right)}{\mathbf{e}\left(\mathcal{N}_{\Gamma ; e}^{\circ}\right)}\right),
$$

and $\left.c_{1}\left(L_{e ; v}\right)\right|_{\mathcal{Z}_{\Gamma ; e}^{\prime \pi}}=-\psi_{e ; v}$, the claim follows from (15.37) and (5.38).

\subsection{Proof of Proposition 5.5}

Let $n^{\prime}=n-k$,

$$
c=\left\{\begin{array}{ll}
\tau, & \text { if } \phi=\tau_{n}^{\prime} ; \\
\eta, & \text { if } \phi=\eta_{n} ;
\end{array} \quad \text { and } \quad G_{c}=\operatorname{Aut}\left(\mathbb{P}^{1}, c\right) .\right.
$$

The 3-dimensional Lie group $G_{c}$ is oriented by the positive rotation around $0 \in \mathbb{P}^{1}$ and the complex orientation of $T_{0} \mathbb{P}^{1}$; see [12, Section 1.4]. This choice of orientation is not directly relevant to the present proof, as it is contained in the formulas from [3] we cite. Denote by $\mathbb{P}^{n^{\prime}-1} \subset \mathbb{P}^{n-1}$ the span of the first $n^{\prime}$ homogenous coordinates and by

$$
\left(V_{c}, \varphi_{c}\right) \longrightarrow\left(\mathbb{P}^{n^{\prime}-1}, \phi^{\prime}\right)
$$

where $\phi^{\prime}=\left.\phi\right|_{\mathbb{P} n^{\prime}-1}$, the normal bundle of $\mathbb{P}^{n^{\prime}-1}$ in $\mathbb{P}^{n-1}$. This holomorphic real bundle pair is the restriction of the last $k$ components of the middle term in (2.7) to $\mathbb{P}^{n^{\prime}-1}$. If $(V, \varphi)$ is a real bundle pair over $\left(\mathbb{P}^{1}, c\right)$, let

$$
\Gamma\left(\mathbb{P}^{1} ; V\right)^{\varphi} \equiv\left\{\xi \in \Gamma\left(\mathbb{P}^{1} ; V\right): \xi \circ c=\varphi \circ \xi\right\}
$$

denote the space of real sections.

Let $e=\left\{v_{1}, v_{2}\right\}$ so that $\vartheta\left(v_{1}\right) \notin 2 \mathbb{Z}$ and

$$
\begin{gathered}
\mathfrak{M}_{\Gamma, \sigma ; e}^{\phi, c ;}=\mathfrak{M}_{0,0}\left(\mathbb{P}^{n-1}, \mathfrak{d}(e)\right)^{\phi, c} \\
\mathfrak{M}\left(\mathbb{P}^{n^{\prime}-1}\right)=\mathfrak{M}_{0,0}\left(\mathbb{P}^{n^{\prime}-1}, \mathfrak{d}(e)\right)^{\phi^{\prime}, c}, \quad \mathfrak{M}\left(X_{n ; \mathbf{a}}\right)=\mathfrak{M}_{0,0}\left(X_{n ; \mathbf{a}}, \mathfrak{d}(e)\right)^{\phi_{n ; \mathbf{a}}, c} .
\end{gathered}
$$


By [3, Lemma 5.1], the space

$$
\overline{\mathfrak{M}}_{\Gamma, \sigma ; e}^{\phi ; \mathbb{T} ; \circ}=\mathfrak{M}_{\Gamma, \sigma ; e}^{\phi, c ; \mathbb{T} ; \circ}
$$

consists of one element: the equivalence class of the unmarked real degree $\mathfrak{d}(e)$ covering

$$
f_{0}:\left(\mathbb{P}^{1}, c, 0, \infty\right) \longrightarrow\left(\mathbb{P}_{\vartheta\left(v_{1}\right) \vartheta\left(v_{2}\right)}^{1}, \phi, P_{\vartheta\left(v_{1}\right)}, P_{\vartheta\left(v_{2}\right)}\right)
$$

branched only over $P_{\vartheta\left(v_{1}\right)}$ and $P_{\vartheta\left(v_{2}\right)}=P_{\phi\left(\vartheta\left(v_{1}\right)\right)}$. We denote by

$$
G_{c}\left(f_{0}\right) \subset H^{0}\left(\mathbb{P}^{1} ; f_{0}^{*} T \mathbb{P}_{\vartheta\left(v_{1}\right) \vartheta\left(v_{2}\right)}^{1}\right)^{f_{0}^{*} \mathrm{~d} \phi}
$$

the tangent space to the orbit of the $G_{c}$-action on the space of parametrized branched covers (by the composition with the inverse of each automorphism as usual). The orientation on $G_{c}$ induces an orientation on $G_{c}\left(f_{0}\right)$.

Let $\bar{\partial}_{\mathcal{L}_{n ; \mathbf{a}}}$ denote the standard $\bar{\partial}$-operator on the real bundle pair

$$
f_{0}^{*}\left(\mathcal{L}_{n ; \mathbf{a}}, \widetilde{\phi}_{n ; \mathbf{a}}\right) \longrightarrow\left(\mathbb{P}^{1}, c\right) .
$$

The evaluations of real holomorphic sections and their derivatives at $z=0$ induce a $\mathbb{T}^{m}$-equivariant isomorphism

$$
\left.H^{0}\left(\mathbb{P}^{1} ; f_{0}^{*} \mathcal{L}_{n ; \mathbf{a}}\right)^{f_{0}^{*} \tilde{\phi}_{n ; \mathbf{a}}} \longrightarrow \bigoplus_{i=1}^{k} \bigoplus_{r=0}^{\left(a_{i} \mathrm{~d}(e)-1\right) / 2} \mathcal{O}_{\mathbb{P}^{n-1}}\left(a_{i}\right)\right|_{P_{\vartheta\left(v_{1}\right)}} \otimes\left(T_{0}^{*} \mathbb{P}^{1}\right)^{\otimes r}
$$

The orientation on

$$
\operatorname{det}\left(\bar{\partial}_{\mathcal{L}_{n ; \mathbf{a}}}\right)=\Lambda_{\mathbb{R}}^{\text {top }}\left(H^{0}\left(\mathbb{P}^{1} ; f_{0}^{*} \mathcal{L}_{n ; \mathbf{a}}\right)^{f_{0}^{*} \tilde{\phi}_{n ; \mathbf{a}}}\right)
$$

induced by the isomorphism (5.39) is called the complex orientation in [12, Section 3.3]. Using

$$
\mathbf{e}\left(\left.\mathcal{O}_{\mathbb{P}^{n-1}}\left(a_{i}\right)\right|_{P_{\vartheta\left(v_{1}\right)}}\right)=a_{i} \alpha_{\vartheta\left(v_{1}\right)}, \quad \mathbf{e}\left(T_{0}^{*} \mathbb{P}^{1}\right)=\frac{\alpha_{\vartheta\left(v_{2}\right)}-\alpha_{\vartheta\left(v_{1}\right)}}{\mathfrak{d}(e)}=-\frac{2 \alpha_{\vartheta\left(v_{1}\right)}}{\mathfrak{d}(e)}
$$

we find that

$$
\begin{aligned}
\mathbf{e}\left(H^{0}\left(\mathbb{P}^{1} ; f_{0}^{*} \mathcal{L}_{n ; \mathbf{a}}\right)^{f_{0}^{*} \tilde{\phi}_{n ; \mathbf{a}}}\right) & =\prod_{i=1}^{k} \prod_{r=0}^{\left(a_{i} \mathfrak{d}(e)-1\right) / 2}\left(a_{i} \alpha_{\vartheta\left(v_{1}\right)}-r \frac{2 \alpha_{\vartheta\left(v_{1}\right)}}{\mathfrak{d}(e)}\right) \\
& =\prod_{i=1}^{k}\left(a_{i} \mathfrak{d}(e)\right) ! !\left(\frac{\alpha_{\vartheta\left(v_{1}\right)}}{\mathfrak{d}(e)}\right)^{\frac{|\mathbf{a}| \mathfrak{d}(e)+k}{2}}
\end{aligned}
$$

with respect to the complex orientation; see [18, Section 27.2].

By Section 2.3, the canonical real orientation on $\left(X_{n ; \mathbf{a}}, \phi_{n ; \mathbf{a}}\right)$ does not depend on the ordering of pairs $(2 i-1,2 i)$ of homogeneous coordinates on $\mathbb{P}^{n-1}$. Thus, we can assume that $\vartheta\left(v_{1}\right) \leqslant n^{\prime}$. Denote by $\bar{\partial}_{\mathbb{P}^{\prime}-1}$ and $\bar{\partial}_{V_{c}}$ the standard $\bar{\partial}$-operators on the real bundle pairs

$$
f_{0}^{*}\left(T \mathbb{P}^{n^{\prime}-1}, \mathrm{~d} \phi\right), f_{0}^{*}\left(V_{c}, \varphi_{c}\right) \longrightarrow\left(\mathbb{P}^{1}, c\right),
$$


respectively. Combining

$$
\mathbf{e}\left(T_{P_{\vartheta\left(v_{1}\right)}} \mathbb{P}_{\vartheta\left(v_{1}\right) j}^{1}\right)=\alpha_{\vartheta\left(v_{1}\right)}-\alpha_{j} \quad \forall j \in[n]-\vartheta\left(v_{1}\right)
$$

with the second statement in (5.40), we obtain

$$
\begin{aligned}
\mathbf{e}\left(H^{0}\left(\mathbb{P}^{1} ; f_{0}^{*} V_{c}\right)^{f_{0}^{*} \varphi_{c}}\right) & =\prod_{j=n^{\prime}+1}^{n} \prod_{r=0}^{(\mathfrak{d}(e)-1) / 2}\left(\alpha_{\vartheta\left(v_{1}\right)}-\alpha_{j}-r \frac{2 \alpha_{\vartheta}\left(v_{1}\right)}{\mathfrak{d}(e)}\right) \\
& =\prod_{j=n^{\prime}+1}^{n} \prod_{r=0}^{(\mathfrak{d}(e)-1) / 2}\left(\frac{(\mathfrak{d}(e)-2 r) \alpha_{\vartheta\left(v_{1}\right)}}{\mathfrak{d}(e)}-\alpha_{j}\right)
\end{aligned}
$$

with respect to the complex orientation on the determinant of the standard $\bar{\partial}$-operator on $\left(V_{c}, \varphi_{c}\right)$.

Let

$$
\begin{aligned}
\left.\left.0 \longrightarrow(V, \varphi) \longrightarrow\left(T \mathbb{P}^{n^{\prime}-1},\left.\mathrm{~d} \phi\right|_{T \mathbb{P}^{n^{\prime}-1}}\right)\right|_{\mathbb{P}_{\vartheta\left(v_{1}\right) \vartheta\left(v_{2}\right)}^{1}} \oplus\left(V_{c}, \varphi_{c}\right)\right|_{\mathbb{P}_{\vartheta\left(v_{1}\right) \vartheta\left(v_{2}\right)}} \\
\left.\longrightarrow\left(\mathcal{L}_{n ; \mathbf{a}}, \tilde{\phi}_{n ; \mathbf{a}}\right)\right|_{\mathbb{P}_{\vartheta\left(v_{1}\right) \vartheta\left(v_{2}\right)}^{1}} \longrightarrow 0
\end{aligned}
$$

be an exact sequence of holomorphic real bundle pairs over $\left(\mathbb{P}_{\vartheta\left(v_{1}\right) \vartheta\left(v_{2}\right)}^{1}, \phi\right)$ such that

$$
T \mathbb{P}_{\vartheta\left(v_{1}\right) \vartheta\left(v_{2}\right)}^{1} \subset V .
$$

Let $\bar{\partial}_{V}$ denote the standard $\bar{\partial}$-operator on the real bundle pair

$$
f_{0}^{*}(V, \varphi) \longrightarrow\left(\mathbb{P}^{1}, c\right)
$$

The real Cauchy-Riemann operators $\bar{\partial}_{V}$ and $\bar{\partial}_{\mathbb{P}^{n^{\prime}-1}}$ descend to operators $\bar{\partial}_{V}^{\prime}$ and $\bar{\partial}_{\mathbb{P}^{n^{\prime}-1}}^{\prime}$ on the quotients of their domains by

$$
G_{c}\left(f_{0}\right) \subset H^{0}\left(\mathbb{P}^{1} ; f_{0}^{*} T \mathbb{P}_{\vartheta\left(v_{1}\right) \vartheta\left(v_{2}\right)}^{1}\right)^{f_{0}^{*} \mathrm{~d} \phi} \subset \Gamma\left(\mathbb{P}^{1} ; f_{0}^{*} V\right)^{f_{0}^{*} \varphi}, \Gamma\left(\mathbb{P}^{1} ; f_{0}^{*} T \mathbb{P}^{n^{\prime}-1}\right)^{f_{0}^{*} \mathrm{~d} \phi} .
$$

The exact sequence (5.43) gives rise to an exact sequence

$$
0 \longrightarrow \bar{\partial}_{V}^{\prime} \longrightarrow \bar{\partial}_{\mathbb{P}^{n^{\prime}-1}}^{\prime} \oplus \bar{\partial}_{V_{c}} \longrightarrow \bar{\partial}_{\mathcal{L}_{n ; \mathbf{a}}} \longrightarrow 0
$$

of Fredholm operators over $\left(\mathbb{P}^{1}, c\right)$. The operators $\bar{\partial}_{\mathbb{P n}^{\prime}-1}^{\prime}, \bar{\partial}_{V_{c}}$, and $\bar{\partial}_{\mathcal{L}_{n ; \mathbf{a}}}$ are surjective and the kernel of $\bar{\partial}_{\mathbb{P}^{n^{\prime}-1}}^{\prime}$ is canonically isomorphic to the tangent space of $\mathfrak{M}\left(\mathbb{P}^{n^{\prime}-1}\right)$ at $\left[f_{0}\right]$.

The case $\phi=\tau_{n}^{\prime}$ and $n-|\mathbf{a}| \in 4 \mathbb{Z}$. By Lemma 2.2 , $n^{\prime} \in 4 \mathbb{Z}$. The canonical spin structure on the real locus of

$$
\frac{n^{\prime}}{2}\left(2 \mathcal{O}_{\mathbb{P}^{n-1}}(1), \widetilde{\tau}_{n ; 1,1}^{(1)}\right) \longrightarrow\left(\mathbb{P}^{n-1}, \tau_{n}^{\prime}\right)
$$

as in Section 2.2 and the exact sequence (2.7) determine a spin structure on

$$
\mathbb{R} \mathbb{P}^{n^{\prime}-1}=\operatorname{Fix}\left(\tau_{n^{\prime}}\right) \subset \mathbb{P}^{n^{\prime}-1} .
$$


This is the same spin structure as in [3, Section 5.5]. The equivariant Euler class of the tangent space of $\left[f_{0},(0, \infty)\right]$ in $\mathfrak{M}_{0,1}\left(\mathbb{P}^{n^{\prime}-1}, \mathfrak{d}(e)\right)^{\phi^{\prime}}$ with respect to the orientation induced by this spin structure is provided by [3, Proposition 6.2] with $d_{0}=\mathfrak{d}(e), i=\vartheta\left(v_{1}\right)$, and $\lambda_{j}=\alpha_{j}$. The Euler class of $\left[f_{0}\right]$ in $\mathfrak{M}\left(\mathbb{P}^{n^{\prime}-1}\right)$ is obtained by dividing the expression in [3] by $\mathbf{e}\left(T_{0} \mathbb{P}^{1}\right)$. Thus,

$$
\begin{aligned}
& \left.\mathbf{e}\left(T_{\left[f_{0}\right]} \mathfrak{M}\left(\mathbb{P}^{n^{\prime}-1}\right)\right)=(-1)^{\mathfrak{d}(e)} \mathfrak{d}(e) !\left(\frac{2 \alpha_{\vartheta}\left(v_{1}\right)}{\mathfrak{d}(e)}\right)^{\mathfrak{d}(e)-1} \prod_{\substack{1 \leqslant j \leqslant n^{\prime} \\
j \neq \vartheta\left(v_{1}\right), 2 \mid\left(j-\vartheta\left(v_{1}\right)\right)}} \prod_{\substack{r=0 \\
\mathfrak{d}(e)}}^{\mathfrak{d}(e)-2 r} \frac{\mathrm{d}(e)}{\mathfrak{d}\left(e v_{1}\right)}-\alpha_{j}\right) \\
& =(-1)^{\frac{\mathfrak{d}(e)-1}{2}} 2^{\mathfrak{d}(e)-1} \mathfrak{d}(e) !\left(\frac{\alpha_{\vartheta}\left(v_{1}\right)}{\mathfrak{d}(e)}\right)^{\mathfrak{d}(e)-1} \prod_{\substack{1 \leqslant j \leqslant n^{\prime} \\
j \neq \vartheta\left(v_{1}\right), \vartheta\left(v_{2}\right)}} \prod_{r=0}^{(\mathfrak{d}(e)-1) / 2}\left(\frac{(\mathfrak{d}(e)-2 r) \alpha_{\vartheta\left(v_{1}\right)}}{\mathfrak{d}(e)}-\alpha_{j}\right) .
\end{aligned}
$$

Similarly to Section [2.2, the canonical spin structure on (5.46) induces a trivialization of $V^{\varphi}$ via the exact sequence (5.43) and thus an orientation on $\operatorname{det} \bar{\partial}_{V}^{\prime}$ as in [12, Section 3.3]. By [12, Corollary 3.13], the isomorphism

$$
\begin{aligned}
\left(\operatorname{det} \bar{\partial}_{V}^{\prime}\right) \otimes\left(\operatorname{det}\left(\left.\mathcal{V}_{n ; \mathbf{a}}^{\tilde{\phi}_{n ; \mathbf{a}}}\right|_{\left[f_{0}\right]}\right)\right) & =\left(\operatorname{det} \bar{\partial}_{V}^{\prime}\right) \otimes\left(\operatorname{det} \bar{\partial}_{\mathcal{L}_{n ; \mathbf{a}}}\right) \\
& \approx \Lambda_{\mathbb{R}}^{\operatorname{top}}\left(T_{\left[f_{0}\right]} \mathfrak{M}\left(\mathbb{P}^{n^{\prime}-1}\right)\right) \otimes\left(\operatorname{det} \bar{\partial}_{V_{c}}\right)=\operatorname{det}\left(\left.\mathcal{N}_{\Gamma, \sigma ; e}^{\phi ; \circ}\right|_{\left[f_{0}\right]}\right)
\end{aligned}
$$

induced by (5.44) is orientation-preserving with respect to the orientations on the first terms on the two sides induced by the canonical spin structure on (5.46) and the complex orientations on the other terms. By (5.41), (5.42), and (5.47), (5.27) thus holds with respect to the orientation on the left-hand side induced by the orientation on $\operatorname{det} \bar{\partial}_{V}^{\prime}$ corresponding to the canonical spin structure on the real locus of (5.45) via the exact sequences (2.7) and (5.43).

The orientation on $\mathfrak{M}\left(X_{n ; \mathbf{a}}\right)$ in this case is determined by the distinguished homotopy class of isomorphisms (2.24) with $\ell_{0}(\mathbf{a})=0$ and thus $\ell_{1}(\mathbf{a})=k$. Since the real line bundle $\left(L^{*}\right)^{\tilde{\phi}^{*}}$ is orientable in this case, the distinguished homotopy class of isomorphisms (2.24) determines a homotopy class of isomorphisms

$$
\begin{aligned}
\left.\left(X_{n ; \mathbf{a}}^{\phi_{n} \mathbf{a}} \times \mathbb{R}\right) \oplus T X_{n ; \mathbf{a}}^{\phi_{n ; \mathbf{a}}} \oplus \mathcal{L}_{n ; \mathbf{a}}^{\tilde{\phi}_{n ; \mathbf{a}}}\right|_{X_{n ; \mathbf{a}}^{\phi_{n}}} & \left.\approx\left(X_{n ; \mathbf{a}}^{\phi_{n ; \mathbf{a}}} \times \mathbb{R}\right) \oplus T X_{n ; \mathbf{a}}^{\phi_{n ; \mathbf{a}}} \oplus k \mathcal{O}_{\mathbb{R} \mathbb{P}^{n-1}}(1)\right|_{X_{n ; \mathbf{a}}^{\phi_{n} \mathbf{a}}} \\
& \left.\left.\approx n^{\prime} \mathcal{O}_{\mathbb{R}^{\mathbb{P}-1}}(1)\right|_{X_{n ; \mathbf{a}}^{\phi_{n}}} \oplus k \mathcal{O}_{\mathbb{R}^{n-1}}(1)\right|_{X_{n ; \mathbf{a}}^{\phi_{n} ; \mathbf{a}}}
\end{aligned}
$$

The canonical spin structure on the real locus of (5.45) thus determines a spin structure on $T X_{n ; \mathbf{a}}^{\phi_{n} \text {, }}$, an orientation on $\mathfrak{M}\left(X_{n ; \mathbf{a}}\right)$, and an orientation on the left-hand side of (5.27) via the short exact sequence (2.14) pulled back to $\mathfrak{M}\left(X_{n ; \mathbf{a}}\right)$. Since $\mathfrak{M}_{\Gamma, \sigma ; e}^{\phi, c ;}$ is connected, the last orientation agrees with the orientation described below (5.48). By [12, Theorem 1.5], the orientation on $\mathfrak{M}\left(X_{n ; \mathbf{a}}\right)$ induced by the real orientation of Section 2.2 is the same as the orientation induced by the canonical spin structure on $\left(X_{n ; \mathbf{a}}, \phi_{n ; \mathbf{a}}\right)$. Thus, the orientation on the left-hand side of (5.27) with the respect to the canonical orientation on $\overline{\mathfrak{M}}_{0}\left(X_{n ; \mathbf{a}}, \mathfrak{d}(e)\right)^{\phi_{n} ; \mathbf{a}}$ arising from Section 2.2 is described by the righthand side of (5.27) in this case. 
The case $\phi=\tau_{n}^{\prime}$ and $n-|\mathbf{a}| \notin 4 \mathbb{Z}$. By Lemma 2.2, $n^{\prime}+2 \in 4 \mathbb{Z}$. The canonical spin structure on the real locus of

$$
\frac{n^{\prime}+2}{2}\left(2 \mathcal{O}_{\mathbb{P}^{n-1}}(1), \widetilde{\tau}_{n ; 1,1}^{(1)}\right) \longrightarrow\left(\mathbb{P}^{n-1}, \tau_{n}^{\prime}\right)
$$

as in Section 2.2 and the exact sequence (2.7) determine a relative spin structure on (5.46). This is the same relative spin structure as in [3, Remark 6.5]. The equivariant Euler class of the tangent space of $\left[f_{0},(0, \infty)\right]$ in $\mathfrak{M}_{0,1}\left(\mathbb{P}^{n^{\prime}-1}, \mathfrak{d}(e)\right)^{\tau_{n^{\prime}}^{\prime}}$ with respect to the orientation induced by this relative spin structure is provided by [3, (6.21)]; see [3, Remark 6.6]. Thus,

$$
\left.\mathbf{e}\left(T_{\left[f_{0}\right]} \mathfrak{M}_{0}\left(\mathbb{P}^{n^{\prime}-1}\right)\right)=2^{\mathfrak{d}(e)-1} \mathfrak{d}(e) !\left(\frac{\alpha_{\vartheta}\left(v_{1}\right)}{\mathfrak{d}(e)}\right)^{\mathfrak{d}(e)-1} \prod_{\substack{1 \leqslant j \leqslant n^{\prime} \\ j \neq \vartheta\left(v_{1}\right), \vartheta\left(v_{2}\right)}}^{(\mathfrak{d}(e)-1) / 2} \prod_{\substack{r=0 \\ j(e)}}^{(\mathfrak{d}(e)-2 r) \alpha_{\vartheta\left(v_{1}\right)}}-\alpha_{j}\right)
$$

The canonical relative spin structure on (5.46) induces a relative spin structure on $V^{\varphi}$ via the exact sequence (5.43) and thus an orientation on $\operatorname{det} \bar{\partial}_{V}^{\prime}$ as in [12, Section 3.3]. By [12, Corollary 3.13], the isomorphism (5.48) induced by (5.44) is orientation-preserving with respect to the orientations on the first terms on the two sides induced by the canonical relative spin structure on (5.46) and the complex orientations on the other terms. By (5.41), (5.42), and (5.50), (5.27) without the leading sign in (4.23) thus holds with respect to the orientation on the left-hand side induced by the orientation on $\operatorname{det} \bar{\partial}_{V}^{\prime}$ corresponding to the canonical relative spin structure on the real locus of (5.49) via the exact sequences (2.7) and (5.43).

The orientation on $\mathfrak{M}\left(X_{n ; \mathbf{a}}\right)$ in this case is determined by the distinguished homotopy class of isomorphisms (2.25) with $\ell_{0}(\mathbf{a})=0$ and thus $\ell_{1}(\mathbf{a})=k$. Since the real line bundle $\left(L^{*}\right)^{*}$ is not orientable in this case, the distinguished homotopy class of isomorphisms (2.25) determines a homotopy class of isomorphisms

$$
\begin{aligned}
& \left.\left(X_{n ; \mathbf{a}}^{\phi_{n} \mathbf{a}} \times \mathbb{R}\right) \oplus\left(\left.T X_{n ; \mathbf{a}}^{\phi_{n}} \oplus 2\left(L^{*}\right)^{\tilde{\phi}^{*}}\right|_{X_{n ; \mathbf{a}}^{\phi_{n}}}\right) \oplus \mathcal{L}_{n ; \mathbf{a}}^{\widetilde{\phi}_{n ; \mathbf{a}}}\right|_{X_{n ; \mathbf{a}}^{\phi_{n}}} \\
& \left.\approx\left(X_{n ; \mathbf{a}}^{\phi_{n ; \mathbf{a}}} \times \mathbb{R}\right) \oplus\left(\left.T X_{n ; \mathbf{a}}^{\phi_{n ; \mathbf{a}}} \oplus 2 \mathcal{O}_{\mathbb{R}^{n-1}}(1)\right|_{X_{n ; \mathbf{a}}^{\phi_{n} ; \mathbf{a}}}\right) \oplus k \mathcal{O}_{\mathbb{R}^{n-1}}(1)\right|_{X_{n ; \mathbf{a}}^{\phi_{n} ; \mathbf{a}}} \\
& \left.\left.\approx\left(n^{\prime}+2\right) \mathcal{O}_{\mathbb{R}^{n-1}}(1)\right|_{X_{n ; \mathbf{a}}^{\phi_{n} ; \mathbf{a}}} \oplus k \mathcal{O}_{\mathbb{R}^{n-1}}(1)\right|_{X_{n ; \mathbf{a}}^{\phi_{n} ; \mathbf{a}}} .
\end{aligned}
$$

The canonical spin structure on the real locus of (5.49) thus determines a relative spin structure on $T X_{n ; \mathbf{a}}^{\phi_{n} \mathbf{a}}$, an orientation on $\mathfrak{M}\left(X_{n ; \mathbf{a}}\right)$, and an orientation on the left-hand side of (5.27) via the short exact sequence (2.14) pulled back to $\mathfrak{M}\left(X_{n ; \mathbf{a}}\right)$. Since $\mathfrak{M}_{\Gamma, \sigma ; e}^{\phi, c ; \circ}$ is connected (unless $n=2$ ), the last orientation agrees with the orientation described at the end of the previous paragraph.

By [12, Theorem 1.5], the orientation on $\mathfrak{M}\left(X_{n ; \mathbf{a}}\right)$ induced by the real orientation of Section 2.2 differs from the orientation induced by the associated relative spin structure on $\left(X_{n ; \mathbf{a}}, \phi_{n ; \mathbf{a}}\right)$ by $(-1)$ to the power of

$$
\left\lfloor\frac{\left\langle c_{1}\left(X_{n ; \mathbf{a}}\right), \mathfrak{d}(e) \ell\right\rangle+2}{4}\right\rfloor=\frac{(n-|\mathbf{a}|) \mathfrak{d}(e)+2}{4},
$$

where $\ell \in H_{2}\left(\mathbb{P}^{n-1} ; \mathbb{Z}\right)$ is the homology class of a line. Replacing the real bundle bundle pair $\left(L^{*}, \tilde{\phi}^{*}\right)$ with $\left(\mathcal{O}_{\mathbb{P}^{n-1}}(1), \widetilde{\tau}_{n ; 1}^{\prime}\right)$ above, we obtain the relative spin structure on $\left(X_{n ; \mathbf{a}}, \phi_{n ; \mathbf{a}}\right)$ induced by the 
canonical relative spin structure on (5.46). The orientations on $\mathfrak{M}_{0}\left(X_{n ; \mathbf{a}}, \mathfrak{d}(e)\right)^{\phi_{n ; \mathbf{a}}, c}$ induced by the two relative spin structures differ by $(-1)$ to the power of

$$
\frac{1}{2}\left\langle 1-c_{1}\left(L^{*}\right), \mathfrak{d}(e) \ell\right\rangle=\frac{(n-|\mathbf{a}|) \mathfrak{d}(e)+2 \mathfrak{d}(e)}{4} .
$$

Adding up the right-hand sides of the last two equations, we conclude the orientation on $\mathfrak{M}\left(X_{n ; \mathbf{a}}\right)$ induced by the real orientation of Section 2.2 differs from the orientation induced by the canonical relative spin structure by the leading sign in (4.23). Thus, the orientation on the left-hand side of (5.27) with respect to the canonical orientation on $\overline{\mathfrak{M}}_{0}\left(X_{n ; \mathbf{a}}, \mathfrak{d}(e)\right)^{\phi_{n ; \mathbf{a}}}$ arising from Section 2.2 is described by the right-hand side of (5.27) in this case as well.

The case $\phi=\eta_{n}$. The top exterior power of the real bundle pair

$$
\frac{n^{\prime}}{2}\left(2 \mathcal{O}_{\mathbb{P}^{n-1}}(1), \eta_{n ; 1,1}^{(1)}\right) \longrightarrow\left(\mathbb{P}^{n-1}, \eta_{n}\right)
$$

is canonically the square of a rank 1 real bundle pair as in Section 2.2. Thus, the restriction of (5.51) to the equator

$$
S_{\vartheta\left(v_{1}\right) \vartheta\left(v_{2}\right)}^{1} \subset \mathbb{P}_{\vartheta\left(v_{1}\right) \vartheta\left(v_{2}\right)}^{1}
$$

has a canonical homotopy class of trivializations. This homotopy class of trivializations determines an orientation on each moduli space $\mathfrak{M}_{0, l}\left(\mathbb{P}^{n^{\prime}-1}, \mathfrak{d}(e)\right)^{\phi^{\prime}}$ as in the proof of [3, Lemma 2.5]. The equivariant Euler class of the tangent space of $\left[f_{0},(0, \infty)\right]$ in $\mathfrak{M}_{0,1}\left(\mathbb{P}^{n^{\prime}-1}, \mathfrak{d}(e)\right)^{\phi^{\prime}}$ with respect to this orientation is again provided by [3, Proposition 6.2] and given by the same expression as in the first case above. Thus,

$$
\begin{aligned}
& \mathbf{e}\left(T_{\left[f_{0}\right]} \mathfrak{M}\left(\mathbb{P}^{n^{\prime}-1}\right)\right) \\
& =(-1)^{\frac{\mathfrak{d}(e)-1}{2}} 2^{\mathfrak{d}(e)-1} \mathfrak{d}(e) !\left(\frac{\alpha_{\vartheta}\left(v_{1}\right)}{\mathfrak{d}(e)}\right)^{\mathfrak{d}(e)-1} \prod_{\substack{1 \leqslant j \leqslant n^{\prime} \\
j \neq \vartheta\left(v_{1}\right), \vartheta\left(v_{2}\right)}}^{(\mathfrak{d}(e)-1) / 2}\left(\frac{(\mathfrak{d}(e)-2 r) \alpha_{\vartheta}\left(v_{1}\right)}{\mathfrak{d}(e)}-\alpha_{j}\right) .
\end{aligned}
$$

The canonical square root structure on (5.51) induces a homotopy class of trivializations of $(V, \varphi)$ over $S_{\vartheta\left(v_{1}\right) \vartheta\left(v_{2}\right)}^{1}$ via the exact sequence (5.43) and thus an orientation on $\operatorname{det} \bar{\partial}_{V}^{\prime}$ as in [12, Section 3.3]. By [12, Corollary 3.16], the isomorphism (5.48) induced by (5.44) is orientation-preserving with respect to the orientations on the first terms on the two sides induced by the canonical square root structure on (5.51) and the complex orientations on the other terms. By (5.41), (5.42), and (5.52), (5.27) without $|\phi|=1$ in the leading exponent thus holds with respect to the orientation on the left-hand side induced by the orientation on $\operatorname{det} \bar{\partial}_{V}^{\prime}$ corresponding to the canonical square root structure on (5.51) via the exact sequences (2.7) and (5.43) and the orienting procedure of [3, Lemma 2.5].

The orientation on $\mathfrak{M}\left(X_{n ; \mathbf{a}}\right)$ in this case is determined by a real orientation on $\left(X_{n ; \mathbf{a}}, \phi_{n ; \mathbf{a}}\right)$ associated with the second isomorphism in (2.18). This isomorphism also determines an orientation on the tangent space of $\mathfrak{M}\left(X_{n ; \mathbf{a}}\right)$ at an element $[u]$ by trivializing $u^{*}\left(T X_{n ; \mathbf{a}}, \phi_{n ; \mathbf{a}}\right)$ along the equator $S^{1} \subset \mathbb{P}^{1}$ as in the proof of [3, Lemma 2.5]. By [12, Corollary 3.8(2)], the two orientations are 
the same. They determine an orientation on the left-hand side of (5.27) via the short exact sequence (2.15) pulled back to $\mathfrak{M}\left(X_{n ; \mathbf{a}}\right)$. Since $\mathfrak{M}_{\Gamma, \sigma ; e}^{\phi, c ; \circ}$ is connected, the last orientation agrees with the orientation described at the end of the previous paragraph. Thus, the orientation on the lefthand side of (5.27) with the respect to the orientation on $\mathfrak{M}\left(X_{n ; \mathbf{a}}\right)$ arising from the canonical real orientation on $\left(X_{n ; \mathbf{a}}, \phi_{n ; \mathbf{a}}\right)$ is given by the right-hand side of (5.27) multiplied by $(-1)^{|\phi|}=(-1)^{|c|}$. As stated at the end of [11, Section 3.2], the canonical orientation of

$$
\mathfrak{M}\left(X_{n ; \mathbf{a}}\right) \equiv \mathfrak{M}_{0,0}\left(X_{n ; \mathbf{a}}, \mathfrak{d}(e)\right)^{\phi_{n ; \mathbf{a}}, c}
$$

with $c=\eta$ is reversed when it is viewed as a subspace of $\overline{\mathfrak{M}}_{0}\left(X_{n ; \mathbf{a}}, \mathfrak{d}(e)\right)^{\phi_{n ; \mathbf{a}}}$. This accounts for the extra factor of $(-1)^{|\phi|}=-1$ in (4.23) in this case.

Institut de Mathématiques de Jussieu - Paris Rive Gauche, Université Pierre et Marie Curie, 4 Place Jussieu, 75252 Paris Cedex 5, France

penka.georgieva@imj-prg.fr

Department of Mathematics, Stony Brook University, Stony Brook, NY 11794

azinger@math.sunysb.edu

\section{References}

[1] M. Atiyah and R. Bott, The moment map and equivariant cohomology, Topology 23 (1984), $1-28$

[2] I. Biswas, J. Huisman, and J. Hurtubise, The moduli space of stable vector bundles over a real algebraic curve, Math. Ann. 347 (2010), no. 1, 201-233

[3] M. Farajzadeh Tehrani, Counting genus zero real curves in symplectic manifolds, math/1205.1809v4, to appear in Geom. Top.

[4] M. Farajzadeh Tehrani, Notes on genus one real Gromov-Witten invariants, math/1406.3786

[5] K. Fukaya and K. Ono, Arnold Conjecture and Gromov-Witten Invariant, Topology 38 (1999), no. $5,933-1048$

[6] K. Fukaya, Y.-G. Oh, H. Ohta, and K. Ono, Lagrangian Intersection Theory: Anomaly and Obstruction, AMS Studies in Advanced Mathematics 46, 2009

[7] A. Gathmann, GROWI, available on the author's website

[8] P. Georgieva, The orientability problem in open Gromov-Witten theory, Geom. Top. 17 (2013), no. $4,2485-2512$

[9] P. Georgieva, Open Gromov-Witten invariants in the presence of an anti-symplectic involution, math/1306.5019v2 
[10] P. Georgieva and A. Zinger, The moduli space of maps with crosscaps: Fredholm theory and orientability, Comm. Anal. Geom. 23 (2015), no. 3, 81-140

[11] P. Georgieva and A. Zinger, Real Gromov-Witten theory in all genera and real enumerative geometry: construction, math/1504.06617v3

[12] P. Georgieva and A. Zinger, Real Gromov-Witten theory in all genera and real enumerative geometry: properties, math/1507.06633v2

[13] P. Georgieva and A. Zinger, Real Gromov-Witten theory in all genera and real enumerative geometry: appendix, available from the authors' websites

[14] E. Getzler, The elliptic Gromov-Witten invariants of $\mathbb{P}^{3}$, math.AG/9612009

[15] T. Graber and R. Pandharipande, Localization of virtual classes, Invent. Math. 135 (1999), no. $2,487-518$

[16] P. Griffiths and J. Harris, Principles of Algebraic Geometry, Wiley, 1994

[17] M. Gromov, Pseudoholomorphic curves in symplectic manifolds, Invent. Math. 82 (1985), no 2, $307-347$

[18] K. Hori, S. Katz, A. Klemm, R. Pandharipande, R. Thomas, C. Vafa, R. Vakil, and E. Zaslow, Mirror Symmetry, Clay Math. Inst., AMS, 2003

[19] J. Kollár, Examples of vanishing Gromov-Witten-Welschinger invariants, math/1401.2387

[20] J. Li and G. Tian, Virtual moduli cycles and Gromov-Witten invariants of general symplectic manifolds, Topics in Symplectic 4-Manifolds, 47-83, First Int. Press Lect. Ser., I, Internat. Press, 1998

[21] J. Li and A. Zinger, On the genus-one Gromov-Witten invariants of complete intersections, J. Differential Geom. 82 (2009), no. 3, 641-690

[22] C.-C. Liu, Moduli of J-holomorphic curves with Lagrangian boundary condition and open Gromov-Witten invariants for an $S^{1}$-pair, math/0210257v2

[23] E. Looijenga, Smooth Deligne-Mumford compactifications by means of Prym level structures, J. Algebraic Geom. 3 (1994), 283-293

[24] D. McDuff and D. Salamon, J-Holomorphic Curves and Quantum Cohomology, University Lecture Series 6, AMS, 1994

[25] J. Milnor and J. Stasheff, Characteristic classes, Annals of Mathematics Studies, No. 76. Princeton University Press, 1974

[26] J. Niu and A. Zinger, Lower bounds for the enumerative geometry of positive-genus real curves, in preparation

[27] J. Niu and A. Zinger, Lower bounds for the enumerative geometry of positive-genus real curves, appendix, available from the authors' websites 
[28] R. Pandharipande, J. Solomon, and J. Walcher, Disk enumeration on the quintic 3-fold, J. Amer. Math. Soc. 21 (2008), no. 4, 1169-1209

[29] A. Popa and A. Zinger, Mirror symmetry for closed, open, and unoriented Gromov-Witten invariants, Adv. Math. 259 (2014), 448-510

[30] Y. Ruan and G. Tian, A mathematical theory of quantum cohomology, J. Differential Geom. 42 (1995), no. 2, 259-367

[31] Y. Ruan and G. Tian, Higher genus symplectic invariants and sigma models coupled with gravity, Invent. Math. 130 (1997), no. 3, 455-516

[32] J. Walcher, Evidence for tadpole cancellation in the topological string, Comm. Number Theory Phys. 3 (2009), no. 1, 111-172

[33] A. Zinger, Enumeration of genus-two curves with a fixed complex structure in $\mathbb{P}^{2}$ and $\mathbb{P}^{3}$, J. Diff. Geom. 65 (2003), no. 3, 341-467

[34] A. Zinger, On the structure of certain natural cones over moduli spaces of genus-one holomorphic maps, Adv. Math. 214 (2007), no. 2, 878-933

[35] A. Zinger, Pseudocycles and integral homology, Trans. AMS 360 (2008), no. 5, 2741-2765

[36] A. Zinger, Standard vs. reduced genus-one Gromov-Witten invariants, Geom. Top. 12 (2008), no. 2, 1203-1241

[37] A. Zinger, A sharp compactness theorem for genus-one pseudo-holomorphic maps, Geom. Top. 13 (2009), no. 5, 2427-2522

[38] A. Zinger, Reduced genus-one Gromov-Witten invariants J. Differential Geom. 83 (2009), no. 2, $407-460$

[39] A. Zinger, A comparison theorem for Gromov-Witten invariants in the symplectic category, Adv. Math. 228 (2011), no. 1, 535-574 